Aus der Klinik für Psychiatrie und Psychotherapie

(Andreas Dally)

des ASKLEPIOS Fachklinikums Tiefenbrunn

\title{
Kardiovaskuläre Stressreaktivität und \\ Recovery bei Frauen: Der Einfluss von \\ Major Depression und \\ Kindheitstraumatisierungen
}

\author{
INAUGURAL-DISSERTATION \\ zur Erlangung des Doktorgrades \\ der Medizinischen Fakultät der \\ Georg-August-Universität zu Göttingen
}

vorgelegt von

Nora Leonie Hermes

aus

Kassel

Göttingen 2019 
Dekan:

\section{Betreuungsausschuss}

Betreuer

Ko-Betreuer:

\section{Prüfungskommission}

Referent

Ko-Referent/in:

Drittreferent/in:

Datum der mündlichen Prüfung:
Prof. Dr. med. W. Brück

Prof. Dr. med. C. Spitzer

Prof. Dr. med. Ch. Herrmann-Lingen
Prof. Dr. med. C. Spitzer

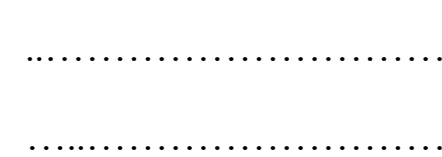


Hiermit erkläre ich, die Dissertation mit dem Titel „Kardiovaskuläre Stressreaktivität und Recovery bei Frauen: Der Einfluss von Major Depression und Kindheitstraumatisierungen" eigenständig angefertigt und keine anderen als die von mir angegebenen Quellen und Hilfsmittel verwendet zu haben.

Göttingen, den 08.10.2019 


\section{Inhaltsverzeichnis}

ABKÜRZUNGSVERZEICHNIS ................................................................ III

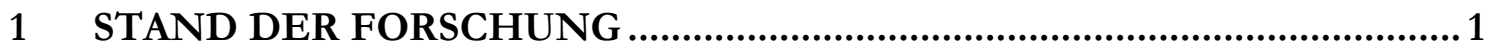

$1.1 \quad$ Kardiovaskuläre Erkrankungen.......................................................................................... 1

1.2 Psychosoziale Risikofaktoren in der Genese kardiovaskulärer Erkrankungen.....................2

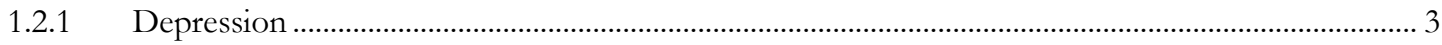

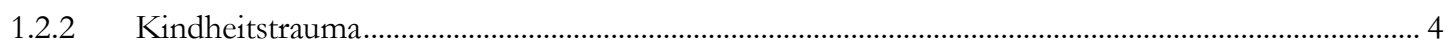

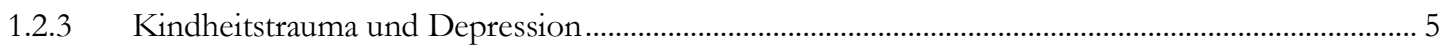

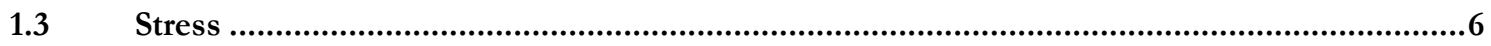

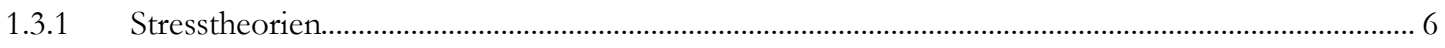

1.3.2 Die Rolle des autonomen Nervensystems innerhalb der kardiovaskulären Stressreaktion .............. 8

1.3.3 Die kardiovaskuläre Stressreaktion im experimentellen Setting ...................................................... 10

1.3.4 Die kardiovaskuläre Stressreaktion und kardiovaskuläre Erkrankungen......................................... 12

1.3.5 Einflussfaktoren auf die kardiovaskuläre Stressreaktion..................................................................... 12

1.3.6 Depression, Kindheitstrauma und die kardiovaskuläre Stressreaktion ............................................ 14

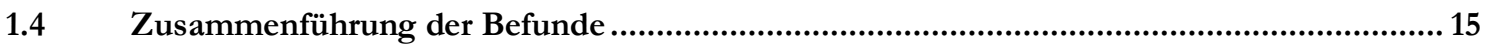

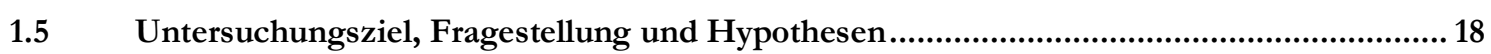

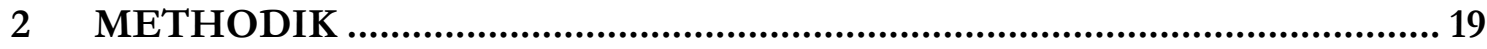

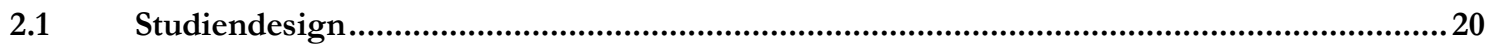

2.1.1 Aufbau und Ablauf der Studie......................................................................................................... 20

2.1.2 Experimentelle Stressinduktion - der Trier Social Stress Test ........................................................ 22

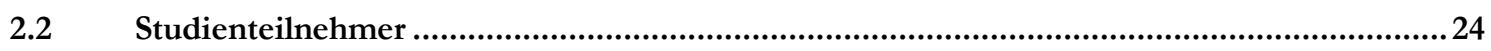

2.2.1 Allgemeine Ein- und Ausschlusskriterien...................................................................................... 24

2.2.2 Spezifische Ein- und Ausschlusskriterien.................................................................................... 25

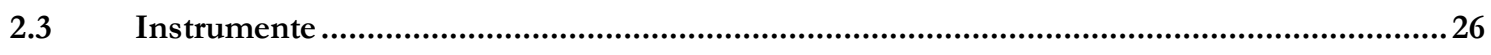

2.3.1 Diagnostische Interviews und Fragebögen .................................................................................... 26

2.3.2 Erfassung physiologischer Parameter................................................................................................... 30

2.4 Datenverarbeitung und statistische Auswertung ..................................................... 31

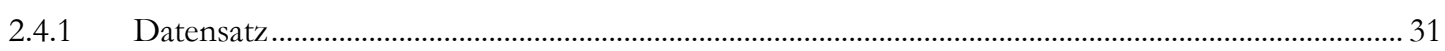

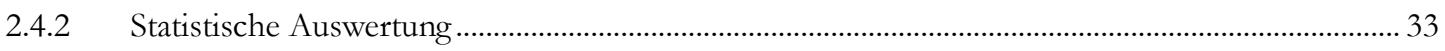

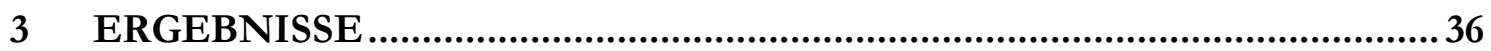

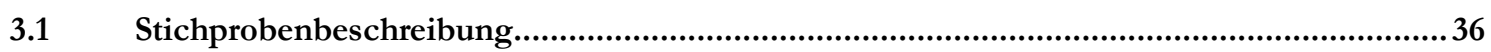




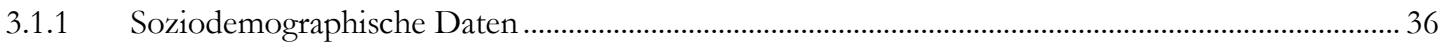

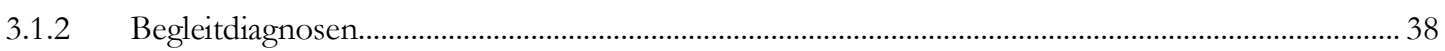

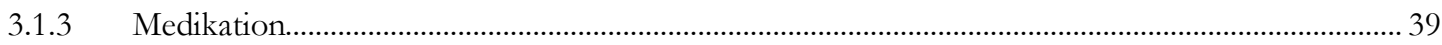

3.1.4 Frühkindliche Traumatisierung und Depressivität............................................................................ 41

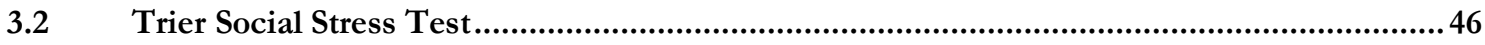

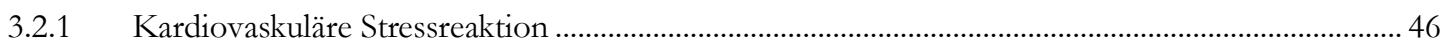

3.2.2 Überprüfen der Hypothesen: Einfluss von Depression und Kindheitstrauma auf die kardiovaskuläre Stressreaktivität und Recovery................................................................................ 51

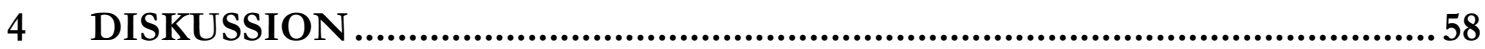

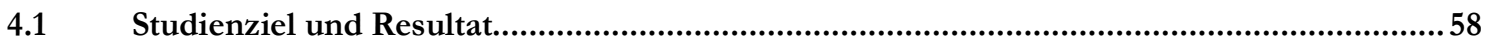

4.2 Einordnung der Ergebnisse in den aktuellen Forschungsstand ......................................59

4.2.1 1. Hypothese: Depression, Kindheitstrauma und kardiovaskuläre Stressreaktivität........................ 59

4.2.2 2. Hypothese: Depression, Kindheitstrauma und kardiovaskuläre Recovery .................................... 66

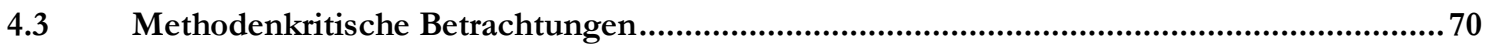

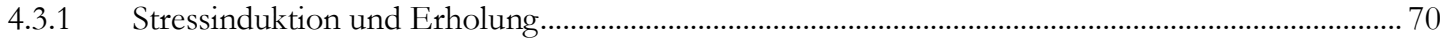

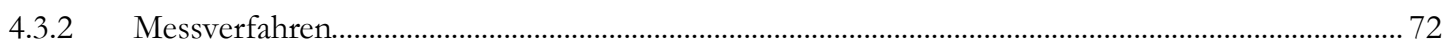

4.3.3 Studienkohorte, soziodemographische und klinische Betrachtungen ........................................... 78

4.3.4 Depression und Kindheitstraumatisierung........................................................................................... 81

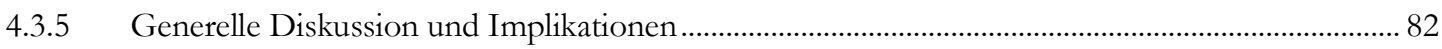

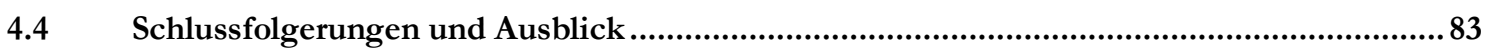

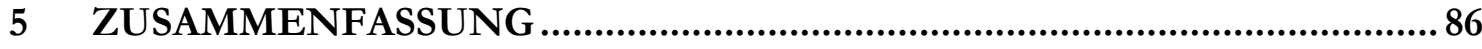

6 ABBILDUNGSVERZEICHNIS .................................................... 88

7 TABELLENVERZEICHNIS .......................................................... 89

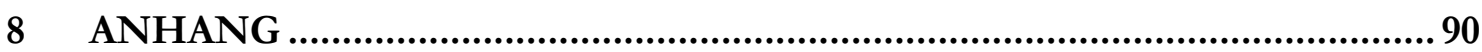

9 LITERATURVERZEICHNIS ........................................................... 92 


\section{Abkürzungsverzeichnis}

ACE

adverse childhood experiences (Kindheitstraumatisierungen), entspricht in dieser Dissertation auch der Versuchsgruppe mental gesunder Probandinnen mit Kindheitstraumatisierungen

ACTH Adenocorticotropin

$\mathrm{ADH}$

Antidiuretisches Hormon

ADR

Adrenalin

aHTN

arterielle Hypertonie

ANS

autonomes Nervensystem

BD

Blutdruck

BDI

Beck Depressions-Inventar

$\mathrm{BL}$

Baseline

BMI

Body-Mass-Index

BRS

Baroreflexsensibilität

CES-D

Center for Epidemiological Science Depression Scale

CI

cardiac index (Herzindex)

$\mathrm{CO}$

cardiac output (Herzzeitvolumen)

$\mathrm{CON}$

Kontrollgruppe, entspricht in dieser Dissertation der Versuchsgruppe

mental gesunder Probandinnen ohne Kindheitstraumatisierungen

$\mathrm{CRH}$

corticotropin-releasing hormone (Corticoliberin)

CTQ

Child Trauma Questionnaire

$\mathrm{dBD}$

diastolischer Blutdruck

DSM

Diagnostic and Statistical Manual of Mental Disorders (diagnostischer und statistischer Leitfaden psychischer Störungen)

DST Dexamethason-Hemmtest

EE emotionale Erfahrungen

EKB erzieherische/körperliche Bestrafung

ELS early life stress (frühkindlicher Stress)

EM emotionaler Missbrauch

ETI Early Trauma Inventory

EV emotionale Vernachlässigung

GES Gesamtskala 
GR Glukocorticoid-Rezeptoren

GT generelle Traumata

HADS Hamilton Anxiety and Depression Scale

HF

Herzfrequenz

HHNA Hypothalamus-Hypophysen-Nebennierenrinden-Achse

HRV Herzratenvariabilität

HZV Herzzeitvolumen

ICD-10 International Statistical Classification of Diseases and Related Health Problems, 10. Revision

KM körperliche Misshandlung

KV körperliche Vernachlässigung

KVE kardiovaskuläre Erkrankungen

KVR kardiovaskuläre Reaktivität

KVRec kardiovaskuläre Recovery

MADRS Montgomery-Åsberg Depression Rating Scale

MAP mean arterial pressure (mittlerer arterieller Druck)

MDD major depressive disorder (Major Depression)

MDD+ major depressive disorder + , entspricht in dieser Dissertation auch der Versuchsgruppe depressiver Patientinnen mit Kindheitstraumatisierungen

MDDmajor depressive disorder -, entspricht in dieser Dissertation auch der Versuchsgruppe depressiver Patientinnen ohne Kindheitstraumatisierungen

MHPG

3-Methoxy-4-Hydroxy-Phenoglykol

MIST

Montreal Imaging Stress Task

MSTT

Mirror Star Tracing Task

NA

Noradrenalin

NDRI

norepinephrine-dopamine reuptake inbibitor (Norepinephrin-Dopamin-

Wiederaufnahmehemmer)

PASAT Paced Auditory Serial Addition Test

PEP Präejektionszeit

PLC Placebogruppe

PNS parasympathisches Nervensystem

PTBS Posttraumatische Belastungsstörung

P-TSST Placebo-Trier Social Stress Test

Q-Q-Plots Quantile-Quantile-Plots

RKI Robert Koch-Institut 
RSA

sBD

SCR

SE

SKID

SM

SNA

SNS

SECPT

SSRI

SSNRI

SV

$\mathrm{t}$

TPR

TPR-I

TSST

TZA

VNS

WHO

ZNS

ZVK respiratorische Sinusarrhythmie

systolischer Blutdruck

skin conductance response (elektrodermale Aktivität)

sexuelle Erlebnisse

Strukturiertes Klinisches Interview für DSM-IV

sexueller Missbrauch

Sympathikus-Nebennierenmark-Achse

sympathisches Nervensystem

socially evaluative cold pressor test (Kaltwasser-Stresstest)

selective serotonin reuptake inbibitor (selektive Serotonin-Wiederaufnahmehemmer)

selective serotonin noradrenalin reuptake inbibitor (selektive Serotonin-Noradrenalin-

Wiederaufnahmehemmer)

Schlagvolumen

time (hier: Messzeitpunkt)

total periphere resistance (totaler peripherer Widerstand)

total periphere resistance index (totaler peripherer Widerstandsindex)

Trier Social Stress Test

trizyklisches Antidepressivum

vegetatives Nervensystem

World Health Organization (Weltgesundheitsorganisation)

zentrales Nervensystem

zerebrovaskuläre Erkrankungen 


\section{Stand der Forschung}

\subsection{Kardiovaskuläre Erkrankungen}

Laut ICD-10 (ICD-10-GM 2019, Kapitel IX, Ziffer 100-199) umfassen kardiovaskuläre Erkrankungen (KVE) alle Störungen des Herz- und Kreislaufsystems, u. a. ischämische und rheumatische Herzkrankheiten, zerebrovaskuläre Erkrankungen (ZVK) und die arterielle Hypertonie (aHTN). Aus einer aktuellen Studie des Robert Koch-Instituts (RKI) geht hervor, dass KVE im Vergleich zu anderen Erkrankungen die höchsten Kosten für das deutsche Gesundheitssystem verursachen und zudem die häufigste Todesursache hierzulande darstellen (Robert Koch-Institut 2015). In Deutschland leidet jeder dritte Erwachsene an hypertensiven Blutdruckwerten (Robert Koch-Institut 2015), welche nach der Weltgesundheitsorganisation (World Health Organization, kurz: WHO) über einen systolischen Blutdruck (sBD) $\geq 140 \mathrm{mmHg}$ und/oder einen diastolischen Blutdruck (dBD) $\geq 90 \mathrm{mmHg}$ definiert sind (WHO 1999a).

Zusammen mit dem metabolischen Syndrom gilt die aHTN als potenter Risikofaktor zur Entwicklung weiterer kardiovaskulärer bzw. arteriosklerotischer Folgeerkrankungen (z. B. ischämische Herzkrankheit, ZVK, periphere arterielle Verschlusskrankheit, linksventrikuläre Hypertrophie und chronische Herzinsuffizienz) und stellt somit ein erhebliches Gesundheitsrisiko dar (Lewington et al. 2002; von Känel 2011).

Die Pathogenese von KVE im Allgemeinen und der aHTN im Speziellen ist nur über multifaktorielle Wege erklärbar. Zu den klassischen Risikofaktoren zählen zum einen nicht beeinflussbare Umstände wie Alter und männliches Geschlecht; zum anderen modifizierbare Einflüsse, welche u. a. einen niedrigen sozioökonomischen Status und ungünstige Ernährungs- und Lebensbedingungen wie Adipositas, Rauchen, einen hohen Salz- und Alkoholkonsum sowie Bewegungsmangel einschließen (Carretero und Oparil 2000; Spitzer et al. 2013; Robert Koch-Institut 2015).

Gleichzeitig wird auch psychosozialen Determinanten bei der Entstehung, Aufrechterhaltung und Therapie kardiovaskulärer Erkrankungen eine bedeutende Rolle zugesprochen (z. B. Herrmann-Lingen 2000; Kaplan und Nunes 2003; Dong et al. 2004; Kinzie et al. 2008; Barth et al. 2010; Low et al. 2010; von Känel 2011; Spitzer et al. 2013; 2016). 


\subsection{Psychosoziale Risikofaktoren in der Genese kardiovaskulärer Erkrankungen}

Wie bereits oben erwähnt, ist der Einfluss psychosozialer Faktoren in der Genese kardiovaskulärer Erkrankungen unumstritten (siehe Kapitel 1.1). So konnte in der renommierten INTERHEART-Studie (Rosengren et al. 2004; Yusuf et al. 2004) gezeigt werden, dass psychosoziale Aspekte (operationalisiert über Depressivität, empfundenen Stress und Lebensereignisse) nach der Dyslipidämie den zweitwichtigsten Risikofaktor zur Entwicklung eines Myokardinfarkts darstellen. Zudem konnten die Autoren nachweisen, dass bei den einzelnen Patienten oft mehrere psychische Risikofaktoren zusammen auftreten.

Psychosoziale Risikofaktoren für KVE lassen sich nach folgenden Aspekten gliedern (Rozanski et al. 2005; von Känel 2008; 2011):

- negative Affektivität (psychologischer Disstress), z. B. Depression, posttraumatischer Stress nach psychologischer Traumatisierung, Trauer

- Persönlichkeitsfaktoren, z. B. Ärgerbereitschaft, Feindseligkeit, Typ-DPersönlichkeit

- soziales Umfeld, z. B. niedriger sozioökonomischer Status, Stress am Arbeitsplatz, niedriger sozialer Support

- Fatigue-Zustände, z. B. vitale Erschöpfung, Insomnie, Burnout

Chronischer psychosozialer Stress kann sich prinzipiell über drei Wege auf das HerzKreislaufsystem auswirken (Spitzer et al. 2013): 1) Förderung eines ungünstigen Lebensstils (Ladwig et al. 2013), 2) Beeinträchtigung der Compliance in der Therapie der KVE (Conraads et al. 2012; Ladwig et al. 2013), 3) direkte pathophysiologische Auswirkungen (Whooley 2006; Pickering 2007; von Känel 2008; 2011). Die vorliegende Arbeit konzentriert sich auf den dritten Weg, wobei psychophysiologische Aspekte in der Pathogenese der KVE bzw. der aHTN besondere Beachtung erfahren sollen. Dabei wird davon ausgegangen, dass psychosozialer Stress langfristig zu einer Modulation im limbischen System führt, wodurch es nachfolgend zu einer veränderten Aktivität des autonomen Nervensystems (ANS) kommt. Infolgedessen werden physiologische Abläufe im HerzKreislaufsystem gestört, was zu einer veränderten kardiovaskulären Sensibilität für Stress führen kann und die Entstehung der KVE im Allgemeinen und der aHTN im Speziellen begünstigt (Pickering 2007; von Känel 2011). In dieser Dissertation soll der Einfluss der psychosozialen Faktoren Depression, Kindheitstrauma sowie des gemeinsamen Auftretens beider Determinanten auf die kardiovaskuläre Stressreaktion untersucht werden. 


\subsubsection{Depression}

Die Depression gehört zu der Gruppe der affektiven Störungen, welche durch eine Veränderung der Stimmungslage und einen Wechsel des Aktivitätsniveaus charakterisiert ist (ICD-10-GM 2019, Kapitel V, Ziffer F30-F39). Die Hauptsymptome sind eine depressive Verstimmung, der Verlust von Interesse/Freude und/oder eine erhöhte Ermüdbarkeit. Zudem können u. a. folgende Nebensymptome auftreten: Herabgesetzte Konzentration, vermindertes Selbstwertgefühl oder Selbstvertrauen, Schlafstörungen, reduzierter Appetit und Suizidgedanken (ICD-10-GM 2019). Laut ICD-10 (Ziffer F32.2) ist die Diagnose einer schweren Depression (engl.: major depression disorder, kurz: MDD) zu stellen, wenn mindestens zwei Haupt- und vier Nebensymptome erfüllt sind. Zum Screening und zur Verlaufsdiagnostik von Depressionen haben sich zudem einige psychometrische Tests zur Selbstund Fremdbeurteilung bewährt, u. a. das Beck Depressions-Inventar (BDI; Beck et al. 1996; Hautzinger et al. 2009) und die Montgomery-Åsberg Depression Rating Scale (MADRS; Montgomery und Åsberg 1979; Williams und Kobak 2008) (AWMF 2015). Die Ätiopathogenese depressiver Erkrankungen stellt ein multifaktorielles Geschehen dar, wobei nach dem Vulnerabilitäts-Stress-Coping-Modell davon ausgegangen wird, dass neben genetischen Prädispositionen auch (psychosoziale) Stressoren wie frühkindliche Traumatisierungen das Risiko zur Entstehung einer depressiven Erkrankung erhöhen (Grabe et al. 2013) (siehe Kapitel 4.3.4). Eine aktuelle, bevölkerungsrepräsentative Studie $(n=5.318)$ kam zu dem Ergebnis, dass die 12-Monats-Prävalenz von Depressionen in Deutschland im Alter von 18 bis 64 Jahren bei Frauen 13,1 \% und bei Männern 6,4 \% beträgt (Jacobi et al. 2014). Leitliniengerechte Therapieoptionen zur Behandlung von unipolaren Depressionen sind u. a. die Pharmakotherapie, z. B. durch trizyklische Antidepressiva (TZA), selektive Serotonin-Wiederaufnahmehemmer (engl.: selective serotonin reuptake inhibitor, kurz: SSRI) und Monoaminoxidase-Inhibitoren (MAO-Hemmer), sowie auch die Psychotherapie (z. B. Verhaltenstherapie, tiefenpsychologisch und analytisch fundierte Gesprächstherapie) und weitere nicht-medikamentöse somatische Therapieverfahren (z. B. elektrokonvulsive Therapie, Wach- und Lichttherapie) (AWMF 2015).

Depressionen stellen insofern eine besondere Herausforderung dar, als dass sie häufig zusammen mit weiteren psychischen und körperlichen Erkrankungen auftreten (Robert Koch-Institut 2015). Eine positive Korrelation von Depressionen und verschiedenen HerzKreislauferkrankungen (Rosengren et al. 2004; Yusuf et al. 2004; Frasure-Smith und Lesperance 2005; Pan et al. 2011; Colquhoun et al. 2013; Whooley und Wong 2013), im Speziellen auch der aHTN (Meng et al. 2012; Kuehl et al. 2016), konnte vielfach belegt werden. 


\subsubsection{Kindheitstrauma}

Traumatisierungen in Kindheit und Jugend werden mittlerweile als ein zentrales Problem für Gesundheit und Gemeinwohl angesehen (Becker und Schulz 2013). Die wissenschaftliche Betrachtung wird jedoch dadurch erschwert, dass bisher keine einheitliche Terminologie und Definition vorliegt (Wingenfeld et al. 2013; Spitzer et al. 2016). So existierten einige gängige, jedoch in ihrer Bedeutung uneinheitliche Begriffe wie biografisch früher Stress, Kindheitstrauma, Kindesmisshandlungen, Kindesmissbrauch und belastende Kindheitserfahrungen (Spitzer et al. 2016). Eine mögliche Definition bietet die WHO (1999b): „Kindesmissbrauch oder -misshandlung umfasst alle Formen der körperlichen und/oder groben emotionalen Misshandlung, des sexuellen Missbrauchs, der Verwahrlosung, der Vernachlässigung oder der kommerziellen bzw. anderweitigen Ausbeutung, die zu einer tatsächlichen oder möglichen Gefährdung der Gesundheit, des Überlebens, der Entwicklung oder der Würde des Kindes führen." Darüber hinaus besteht in der Literatur ein Grundkonsens darüber, dass Kindheitstraumatisierungen aktiv (Missbrauch) oder passiv (Vernachlässigung) ablaufen und sowohl körperliche als auch sexuelle und emotionale Aspekte umfassen können (Wingenfeld et al. 2013; Spitzer et al. 2016). Für den deutschen Sprachraum verfügbare psychometrische Instrumente zur Erfassung frühkindlicher Traumatisierung stellen u. a. der Childhood Trauma Questionnaire (CTQ; Bernstein und Fink 1998; Wingenfeld et al. 2010) und das Early Trauma Inventory (ETI; Bremner et al. 2000; Wingenfeld et al. 2011) dar (Wingenfeld et al. 2013).

In einer deutschen bevölkerungsrepräsentativen Studie ( $n=2.500$; Häuser et al. 2011) wurde zur Umgehung der Problematik hoher Dunkelziffern von Kindheitstraumen in offiziellen Statistiken eine retrospektive Erfassung mithilfe des CTQ durchgeführt. Die Autoren kamen zu dem Ergebnis, dass 68,2\% der Befragten mindestens eine Form der Kindesmisshandlung erfahren hatten; 5,5\% der Teilnehmer berichteten von schwerem bis extremem körperlichen, 6,2 \% von schwerem bis extremem sexuellen Missbrauch. Auch in einer weiteren, aktuellen Allgemeinbevölkerungsstudie $(\mathrm{n}=2.531)$ von Witt und Kollegen (2019) zeigte sich eine hohe Prävalenz von (früh)kindlicher Traumatisierung hierzulande: 43,7 \% der Befragten berichteten von mindestens einem belastenden Kindheitsereignis; 8,9\% gaben an, vier oder mehr belastende Kindheitserfahrungen gemacht zu haben. Diese Zahlen verdeutlichen das Ausmaß dieser Problematik in Deutschland. Die gesellschaftliche Relevanz wird zusätzlich dadurch verschärft, dass frühe Traumatisierungen einen wesentlichen Risikofaktor zur Entstehung negativer Gesundheitsfolgen im Erwachsenenalter darstellen. Eine wegweisende und viel beachtete Studie zu diesem Aspekt stellt die Adverse Childhood Experience(ACE)-Studie (Felitti et al. 1998) dar. In diesem Forschungsprojekt 
wurden über 17.000 erwachsene Versicherte einer US-amerikanischen Krankenversicherung untersucht, wobei mittels zehn Kategorien erfasste traumatische Kindheitserlebnisse (z. B. körperliche Misshandlung, emotionale Vernachlässigung, sexueller Missbrauch, Scheidung der Eltern) in Beziehung zu dem aktuellen körperlichen und psychischen Gesundheitszustand gesetzt wurden. Teilnehmer mit frühem Trauma wiesen häufiger ein risikobehaftetes Gesundheitsverhalten auf, u. a. einen erhöhten Alkohol-, Nikotin- und Medikamentenmissbrauch (Anda et al. 1999, Dube et al. 2006; Anda et al. 2008b), ein riskantes Sexualverhalten (Hillis et al. 2001) und Übergewicht (Williamson et al. 2002). Auch psychische Störungen (z. B. Depression; Edwards et al. 2003; Chapman et al. 2004; Anda et al. 2007) und eine erhöhte Suizidalität (Dube et al. 2001) konnten vermehrt bei traumatisierten Probanden gefunden werden, wobei auf den Zusammenhang zwischen Kindheitstrauma und Depression in einem gesonderten Unterkapitel näher eingegangen werden soll (siehe Kapitel 4.3.4). Die Autoren konnten zudem bei Studienteilnehmern mit belastenden Kindheitserfahrungen vermehrt somatische Erkrankungen nachweisen (u. a. kardiovaskuläre Erkrankungen wie KHK; Dong et al. 2004, und COPD; Anda et al. 2008a). Eine Vielzahl weiterer Studien konnte die positive Korrelation von Kindheitstrauma und HerzKreislauferkrankungen replizieren. Einige Forschungsergebnisse zeigten dabei speziell eine Verbindung von ACE und aHTN auf (Batten et al. 2004; Springer et al. 2007; Riley et al. 2010; Scott et al. 2011; Afifi et al. 2016). Eine aktuelle Studie von Garad und Kollegen (2017) kam zu dem Ergebnis, dass der Zusammenhang zwischen ACE und der Entwicklung von KVE bei Frauen im Vergleich zu Männern besonders stark ausgeprägt ist.

Bei den hier aufgeführten Studienergebnissen muss jedoch beachtet werden, dass die gefundenen Korrelationen zwischen ACE und KVE rein statistischer Natur sind und es noch einer Klärung kausaler Zusammenhänge bedarf (Spitzer et al. 2013).

\subsubsection{Kindheitstrauma und Depression}

Traumatische Lebensereignisse gehen vermehrt mit der Entwicklung psychischer Folgestörungen einher (Fergusson et al. 2013). Eine bekannte und wissenschaftlich viel belegte Traumafolgestörung stellt die Posttraumatische Belastungsstörung (PTBS) dar, welche schätzungsweise 15 bis $50 \%$ der traumatisierten Personen entwickeln (Maercker et al. 2004; Ikin et al. 2010; Grabe et al. 2013). Dennoch scheinen gerade bei in der Kindheit und Jugend traumatisierten Personen andere psychische Folgestörungen zu überwiegen (Grabe et al. 2013), wie affektive Störungen - v.a. depressive Erkrankungen (z. B. Kendler et al. 2000; Bradley et al. 2008; Heim et al. 2008; Ikin et al. 2010; Schoedl et al. 2010; Grabe et al. 2013; Park et al. 2014), Angsterkrankungen (Fergusson et al. 2008; Lindert et al. 2014) und 
dissoziative Störungen (Spitzer und Dümpelmann 2013; Terock et al. 2016; Vonderlin et al. 2018). Im Folgenden soll der Zusammenhang zwischen Kindheitstraumatisierung und depressiven Erkrankungen näher betrachtet werden.

In der bereits oben zitierten ACE-Studie (siehe Kapitel 1.2.2) betrug die Prävalenz depressiver Erkrankungen unter traumatisierten Probanden $23 \%$. Die Autoren konnten zeigen, dass dieser Effekt ,dosisabhängig' war, d. h. je mehr traumatische Ereignisse ein Mensch erlebt hatte (in dieser Studie gemessen über den ACE-Score von 0 bis 10), desto höher war die Wahrscheinlichkeit, im weiteren Verlauf eine Depression zu entwickeln (Chapman et al. 2004). Bei Teilnehmern, die mehrere traumatische Erfahrungen durchlebt hatten (entsprechend einem ACE-Score $\geq 4$ ), betrug die Wahrscheinlichkeit für eine Depression sogar mehr als 50 \%, wobei Frauen eine höhere Gefährdung aufwiesen als Männer.

Eine groß angelegte nationale (Witt et al. 2019) sowie weitere internationale Forschungsprojekte (Putnam 2003; Comijs et al. 2007; Fergusson et al. 2008) konnten die hohe Prävalenz von depressiven Erkrankungen unter (frühkindlich) traumatisierten Probanden replizieren.

Neben lern- und entwicklungstheoretischen Aspekten scheint eine durch neuroendokrine, neurodegenerative und genetische Veränderungen hervorgerufene biologische Vulnerabilität eine Rolle in der Entwicklung von Depressionen bei traumatisierten Personen zu spielen (Bradley et al. 2008; Heim et al. 2008; Grabe et al. 2010; Grabe et al. 2013), worauf jedoch aufgrund der Komplexität an dieser Stelle nicht umfassend eingegangen werden kann.

\subsection{Stress}

In der Genese kardiovaskulärer Erkrankungen und speziell der arteriellen Hypertonie spielen psychosoziale Risikofaktoren eine bedeutende Rolle. Einen möglichen vermittelnden Weg stellt die autonome Dysbalance dar, welche sich in einer veränderten kardiovaskulären Reaktion auf akuten (psychosozialen) Stress äußert.

Im folgenden Kapitel werden die wichtigsten Stresstheorien und die physiologischen Grundlagen der Stressreaktion dargelegt. Zudem soll aufgezeigt werden, wie sich eine veränderte Stressreaktion möglicherweise negativ auf die Gesundheit auswirken könnte.

\subsubsection{Stresstheorien}

Im Laufe des letzten Jahrhunderts wurden zahlreiche Stresstheorien entwickelt. In den folgenden Unterkapiteln wird überblicksartig auf einige gängige Konzepte eingegangen, die eine wichtige theoretische Grundlage für diese Arbeit bilden. 


\subsubsection{Homöostase und Notfallreaktion}

Der Physiologe Walter Cannon (1929; 1975) prägte zu Beginn des 20. Jahrhunderts die Begriffe der Homöostase und der Notfallreaktion (engl.: fight or flight response). Er ging davon aus, dass der Organismus stets über eine Einhaltung vorgegebener Sollwerte der physiologischen Systeme ein konstantes internes Milieu anstrebt. Bei plötzlichen Störungen des Gleichgewichts kommt es zu einer Notfallreaktion. Demnach reagiert der Organismus auf einen akuten Stressor über die Aktivierung des sympathischen Nervensystems (SNS) mit einer schlagartigen Ausschüttung von Adrenalin (ADR). Daraufhin kommt es zu einer immer gleichartig ablaufenden, unspezifischen Reaktion des Körpers, wie z. B. einer Erhöhung der Herzfrequenz (HF) und des Blutdrucks (BD), einer verbesserten Sauerstoffversorgung sowie einer erhöhten Bereitstellung von Glukose. Cannon betrachtete die Notfallreaktion als Stärke des Organismus, Bedrohungen zu entgehen oder sie zu beseitigen.

\subsubsection{Anpassungsreaktion}

Während Cannon (1929; 1975) die Reaktion auf einen akuten Stressor beschrieb, widmete sich der Endokrinologe Hans Selye der Erforschung der chronischen Stresseinwirkung (1936; 1950). Auch Selye beschrieb die Stressreaktion als unspezifisches Antwortverhalten des Organismus auf jegliche Art der Bedrohung. Er teilte die Stressreaktion in drei verschiedene Phasen ein, welche sich durch das Maß der Widerstandskraft gegenüber der Stresseinwirkung unterscheiden: Alarm-, Widerstands- und Erschöpfungsphase. In der Alarmphase reagiert der Körper entsprechend Cannons Theorie der Notfallreaktion kurzfristig über eine Ausschüttung von Katecholaminen auf einen akuten Stressor. Nach Selye kommt es zudem über eine Aktivierung der Hypothalamus-HypophysenNebennierenrinden-Achse (HHNA) zu einer erhöhten Cortisolausschüttung. Dauert die Stressreaktion länger an, mobilisiert der Körper seine Widerstandskräfte und tritt in die zweite Phase ein, wobei über allgemeine Anpassungsmechanismen körperliche Folgen des Stressereignisses gering werden sollen. Erfolgt in der Widerstandsphase keine Regeneration, geht der Organismus nach Verbrauch seiner Reserven in das dritte Stadium, das sogenannte Erschöpfungsstadium, über. Hier kommt es mit einer höheren Wahrscheinlichkeit zur Entstehung von Krankheiten bis hin zum Tod des Individuums (Selye 1950). Selye war der erste Autor, welcher die Begriffe Eustress und Disstress definierte und davon ausging, dass eine gestörte Anpassungsreaktion an Stress negative Gesundheitsfolgen mit sich bringt (Selye 1936; 1975). Zudem beschrieben Cannon und Selye mit der SNA (SympathikusNebennierenmark-Achse) und der HHNA zwei zentrale Stresssysteme, welche immer noch eine wichtige Grundlage in der heutigen Stressforschung darstellen. 


\subsubsection{Allostase und allostatische Last}

McEwen (1998) erweiterte mit seiner Theorie der Allostase und allostatische Last (engl.: allostasis and allostatic load) die klassische Sicht der Homöostase (Cannon 1975) und legte damit einen wichtigen Grundstein der jüngeren Stressforschung. Dieses Stressmodell liefert eine mögliche Erklärung des Zusammenhangs der physiologischen Stressantwort und der Entwicklung von Krankheiten. Laut dem Konzept der Allostase (Sterling und Eyer 1988; McEwen 1998) strebt der Organismus eine Aufrechterhaltung von Stabilität durch Veränderung an. Im Gegensatz zur Theorie der Homöostase gibt es hierbei keine konstanten Sollwerte in den physiologischen Systemen, vielmehr beschreibt die Allostase eine flexible Adaption der physiologischen Systeme an situationsbedingte Veränderungen. Allostatische Regulationen dienen der Leistungsfähigkeit und Funktionstüchtigkeit des Organismus in Extremsituationen (Sterling und Eyer 1988; McEwen 1998). Dysregulierte Zustände wie chronische, wiederholte oder ineffektiv gesteuerte Anpassungen führen zu einer Überlastung der Systeme. Diesen Zustand bezeichnet McEwen (1998) als allostatische Last. Der Autor unterscheidet dabei zwischen drei verschiedenen Typen der Stressantwort, welche allein oder zusammen auftretend zur allostatischen Last führen können:

- Typ I ,frequent stress': Überstimulation der Stresssysteme im Sinne einer zu starken bzw. zu häufigen Stressantwort

- Typ II ,failed shut down': chronische Aktivierung bzw. fehlende Herunterregelung der Stresssysteme

- Typ III ,inadequate response': unzureichende Reaktion eines Stresssystems, wodurch zur Aufrechterhaltung der Stabilität gegenregulierende Systeme überaktiviert werden

Es wird angenommen, dass eine allostatische Überlastung der Stresssysteme zu einer Dysregulation physiologischer Abläufe und somit langfristig zu pathophysiologischen und psychologischen Veränderungen führen kann (McEwen und Seeman 1999; Schulkin 1999; Koob und Le Moal 2001).

\subsubsection{Die Rolle des autonomen Nervensystems innerhalb der kardiovaskulären Stressreaktion}

Durch einen psychosozialen Stressreiz kommt es zunächst zu einer Aktivierung jener Gehirnareale, welche zur Verarbeitung von Emotionen zuständig sind; diese zentrale Funktionseinheit wird limbisches System genannt. Seine Aktivierung führt zu einer Stimulation körpereigener Stresssysteme, wobei das ANS und die HHNA eine Schlüsselrolle in der physiologischen Stressantwort einnehmen. Das ANS ist ein schnelles, flüchtiges System, 
welches innerhalb von Sekunden reagiert, während die im Vergleich eher langsam agierende Reaktionskette der HHNA im Bereich von Minuten ihre Wirkung entfaltet (Koch et al. 2017). Die vorliegende Arbeit fokussiert sich speziell auf die Rolle des ANS; zum allgemeinen Verständnis der physiologischen Stressreaktion soll an dieser Stelle dennoch vorab kurz auf die HHNA eingegangen werden.

Bei einer Stimulation der HHNA kommt es über den Nucleus paraventricularis des Hypothalamus zu einer Ausschüttung von Corticoliberin (engl.: corticotropin-releasing hormone, kurz: $\mathrm{CRH}$ ). CRH bewirkt wiederum die Freisetzung von Adenocorticotropin (ACTH) aus der Hypophyse. Dieses Hormon regt die Nebennierenrinde dazu an, Glukocorticoide, v.a. Cortisol, freizusetzen (Huppelsberg und Walther 2009; Edwards und Mills 2011). Cortisol dient über katabole Stoffwechselprozesse der Mobilisierung körpereigener Energiereserven (Glukoneogenese, Lipo- und Proteolyse) in Stresssituationen. Zudem kommt es u. a. zu einer Sensibilisierung für die Wirkung der Katecholamine an verschiedenen Organen (z. B. an Gefäßen und im Fettgewebe), einer Hemmung von Immunprozessen und einer gesteigerten Erregbarkeit des zentralen Nervensystems (ZNS) (Huppelsberg und Walther 2009). Das ANS teilt sich in zwei Schenkel auf, das sympathische (SNS) und das parasympathische Nervensystem (PNS). Das SNS (,fight or flight') führt zu einer Leistungssteigerung und bereitet den Organismus auf außergewöhnliche Anstrengungen vor (Ergotropie). Das meist dazu antagonistisch wirkende PNS (,rest and digest') dient der Ruhe und Erholung (Trophotropie) des Körpers (Handwerker und Kress 2013). Durch einen physischen oder psychischen Stressreiz kommt es grundsätzlich zur Aktivierung des ANS und einer Inhibition des PNS. Das Stresshormon Noradrenalin (NA) wird aus postganglionären sympathischen Neuronen freigesetzt und induziert eine weitere Ausschüttung von Katecholaminen, überwiegend ADR, aus dem Nebennierenmark in den Blutkreislauf. Von dort aus gelangen die Neurotransmitter an ihre Zielorgane und können über verschiedene $\alpha$ - und $\beta$ adrenerge Rezeptoren ihre Wirkung entfalten. Parasympathische Effekte werden mithilfe des Neurotransmitters Acetylcholin über den Nervus Vagus vermittelt (Handwerker und Kress 2013).

Das kardiovaskuläre System wird sowohl sympathisch als auch vagal reguliert. Das SNS entfaltet seine Wirkung über $\alpha_{1}$-Rezeptoren der glatten Muskulatur von Blutgefäßen und führt zu einer Vasokonstriktion und somit einer Erhöhung des totalen peripheren Widerstands (engl.: total periphere resistance, kurz: TPR)

Am Herzen führen ein erhöhter Sympathikotonus über $\beta_{1}$-Rezeptoren und die gleichzeitige Drosselung des Herzvagus zu einer Erhöhung der HF. Zudem wirkt der Sympathikus in der kardialen Stressreaktion positiv inotrop (Erhöhung der Kontraktionskraft des Herz- 
muskels), dromotrop (schnellere Überleitung der Erregung vom Vorhof auf den Ventrikel), bathmotrop (Erniedrigung der Reizschwelle der Herzmuskulatur) und lusitrop (Erhöhung der Relaxationsgeschwindigkeit). Durch diese Reaktionen kommt es über die veränderte Herzaktivität zu einem erhöhten Schlagvolumen (SV) und Herzzeitvolumen (HZV). Diese dargestellten Mechanismen tragen gemeinsam maßgeblich zur Erhöhung von BD und HF und somit zur Aufrechterhaltung des Herz-Kreislaufsystems im Rahmen von Stresssituationen bei (Rettig und Kuschinsky 2013; Ehmke 2014).

Eine zentrale Rolle in der Regulation kurzfristiger Blutdruckschwankungen nimmt der Barorezeptorreflex ein. Barorezeptoren befinden sich in hoher Dichte im Karotissinus und Aortenbogen. Eine Erhöhung des BD führt über eine vermehrte Dehnung der Gefäßwand zu einer verstärkten Aktivierung der Barorezeptoren, welche durch erhöhte Entladungsfrequenzen codiert wird. In Folge dessen wird die Aktivität der parasympathischen Fasern zum Herzen verstärkt, was zu einer Abnahme der HF und des SV führt. Gleichzeitig wird der periphere Sympathikotonus herabgesetzt und somit der TPR vermindert. Diese Mechanismen führen gemeinsam zu einer Senkung der HF und des BD (Rettig und Kuschinsky 2013).

\subsubsection{Die kardiovaskuläre Stressreaktion im experimentellen Setting}

Wie bereits in Kapitel 1.3.2 beschrieben wurde, reagiert das kardiovaskuläre System sehr sensibel auf psychosoziale Stressreize. Zur Quantifizierung der kardiovaskulären Stressreaktion im experimentellen Setting haben sich mehrere Parameter etabliert, u. a. die HF (Heim et al. 2000; Lovallo et al. 2012; Brindle et al. 2013; Voellmin et al. 2015; Meyer et al. 2016), der dBD und der sBD (de Rooij et al. 2010; Schwerdtfeger und Rosenkaimer 2011; Brindle et al. 2013; Gooding et al. 2016), der mittlere arterielle Blutdruck (engl.: mean arterial pressure, kurz: MAP; Matthews et al. 2005; Meyer et al. 2016), die Präejektionszeit (engl.: pre-eject period, kurz: PEP; Taylor et al. 2006; Salomon et al. 2009; Meyer et al. 2016), die Herzratenvariabilität (HRV; Matthews et al. 2005; Taylor et al. 2006), das Herzzeitvolumen (engl.: cardiac output, kurz: CO; Matthews et al. 2005; Taylor et al. 2006; Salomon et al. 2009; Yuenyongchaiwat et al. 2017) und der TPR (Matthews et al. 2005; Hamer et al. 2007; Meyer et al. 2016).

Diese Arbeit konzentriert sich auf die Auswertung der HF, des sBD und des dBD, da sich diese Parameter sowohl zur Erfassung der kardiovaskulären Stressreaktion als auch zur prädiktiven Risikostratifizierung in der Entwicklung kardiovaskulärer Erkrankungen bewährt haben (Zanstra und Johnston 2011). 
Um eine kardiovaskuläre Stressreaktion unter Versuchsbedingungen auszulösen, wurden einige psychosoziale Stresstests entwickelt, z. B. der Tier Social Stress Test (TSST; Kirschbaum et al. 1993), der Paced Auditory Serial Addition Test (PASAT; Gronwall und Whrightson 1974) und die Montreal Imaging Stress Task (MIST; Dedovic et al. 2005).

In der vorliegenden Arbeit wurde der TSST angewandt. Er ist ein in der Stressforschung etabliertes und standardisiertes Verfahren zur Stressinduktion (Kirschbaum et al. 1993; Dickerson und Kemeny 2004; Kudielka et al. 2004) und bietet dadurch eine gute Vergleichbarkeit mit anderen Studien.

Bei Betrachtung gängiger Studien zur Erfassung der kardiovaskulären Stressreaktion bei früh traumatisierten und depressiven Probanden (Salomon et al. 2009; de Rooij et al. 2010; Schwerdtfeger und Rosenkaimer 2011; Lovallo et al. 2012; Brindle et al. 2013; Voellmin et al. 2015; Gooding et al. 2016) zeigt sich, dass die Stressreaktion zumeist in verschiedene Abschnitte gegliedert wird, wobei die unten aufgeführte Recovery lediglich in einem Teil der Studien betrachtet wird (siehe Kapitel 4.2.2):

- Baseline (BL): Erfasst die kardiovaskulären Ausgangswerte. Die Messung erfolgt zumeist nach einer anfänglichen Ruhephase und vor der experimentellen Stressinduktion.

- kardiovaskuläre Stressreaktivität (KVR): Beschreibt die Antwort der kardiovaskulären Parameter auf den Stressreiz. Dazu werden die kardiovaskulären Marker während bzw. unmittelbar nach der Stresseinwirkung gemessen.

- kardiovaskuläre Recovery (KVRec): Gibt Auskunft über die Erholung der kardiovaskulären Parameter nach der experimentellen Stressinduktion. Die Messung der Parameter erfolgt während bzw. nach einer sich an die Stresseinwirkung anschließenden Erholungsphase. Dabei ist die Recovery in der gängigen Literatur nicht einheitlich operationalisiert. Ein Teil der Autoren betrachtet die Erholung der hämodynamischen Werte in Abhängigkeit von der Stressreaktivität. Dabei wird häufig die Differenz zwischen den gemessenen Werten der Stressreaktivität und den Werten nach der Erholungsphase ermittelt (de Rooij et al. 2010; Voellmin et al. 2015). Größere Werte beschreiben in diesem Fall eine ,größere Erholung'. Wiederum andere Autoren betrachten die Recovery unabhängig von der Stressreaktivität als Erholung der Parameter nach der Erholungsphase im Vergleich zur Baseline (Hamer et al. 2007; Salomon et al. 2009; Schwerdtfeger und Rosenkaimer 2011). Auch hier werden häufig die Differenzen betrachtet; kleinere Werte entsprechen einer ,größeren Erholung' der hämodynamischen Parameter. 


\subsubsection{Die kardiovaskuläre Stressreaktion und kardiovaskuläre Erkrankungen}

Wie bereits oben beschrieben wird angenommen, dass eine autonome Dysbalance über direkte psychoendokrinologische Mechanismen die Entstehung kardiovaskulärer Erkrankungen begünstigt (siehe Kapitel 1.2). Da das ANS maßgeblich an der Steuerung physiologischer Herz-Kreislauf-Funktionen beteiligt ist (siehe Kapitel 1.3.2), liegt es unter Einbeziehung McEwens Theorie der Allostase und allostatischen Last (McEwen 1998; 2002, siehe Kapitel 1.3.1.3) nahe, dass ein möglicher vermittelnder Mechanismus über eine veränderte kardiovaskuläre Stressreaktion führt.

Schon seit Längerem wird eine erhöhte kardiovaskuläre Reaktivität auf psychosozialen Stress als Risikofaktor zur Entwicklung von Herz-Kreislauferkrankungen vermutet (McEwen 2002; Lovallo und Gerin 2003; Schwartz et al. 2003). In einigen prospektiven Studien konnte dieser Zusammenhang nachgewiesen werden: So zeigte sich eine positive Korrelation zwischen einer erhöhten KVR (vorwiegend gemessen über BD und HF) und der Ausbildung einer aHTN (Everson et al. 1996; Markovitz et al. 1998; Newman et al. 1999; Treiber et al. 2003; Matthews et al. 2004; Chida und Steptoe 2010; Carroll et al. 2011; Carroll et al. 2012) sowie weiterer kardiovaskulärer Erkrankungen (Allen et al. 1997; Everson et al. 1997; Jennings et al. 2004; Roemmich et al. 2009; Roemmich et al. 2011). Laut McEwen (1998; 2002) wirkt sich nicht nur eine erhöhte, sondern auch eine prolongierte Stressreaktion im Sinne einer verzögerten Recovery der Stresssysteme negativ auf die Gesundheit aus. Eine gestörte kardiovaskuläre Recovery konnte als ein - von der Stressreaktivität unabhängiger - Risikofaktor zur Entwicklung von KVE identifiziert werden (Schuler und O'Brien 1997; Steptoe und Marmot 2006), welcher u. a. speziell die Entwicklung einer aHTN (Schuler und O’Brien 1997; Treiber et al. 2001; Steptoe und Marmot 2005; Stewart et al. 2006) prädiziert.

\subsubsection{Einflussfaktoren auf die kardiovaskuläre Stressreaktion}

Es gibt einige Aspekte, welche einen Einfluss auf die KVR haben können und daher bei der Auswertung der kardiovaskulären Parameter im experimentellen Setting bedacht werden sollten. Im Folgenden werden einige potentielle Faktoren näher betrachtet.

Einen möglichen Einflussfaktor stellt das Alter der Probanden dar. Die Arbeitsgruppe um Kudielka (2004) führte einen Vergleich des durch den TSST ausgelösten Anstieg der HF zwischen Kindern ( $M=12,3$ Jahre), ,jüngeren’ $(M=23,3$ Jahre) und ,älteren’ Erwachsenen ( $\mathrm{M}=66,9$ Jahre) durch. Die Autoren konnten zeigen, dass Kinder und jüngere Erwachsene einen signifikant höheren Anstieg der HF zeigten als ältere Erwachsene. Dies deckt sich mit den Forschungsergebnissen von Carroll und Kollegen (2007), wobei in diesem Stu- 
dienkollektiv zusätzlich gezeigt werden konnte, dass das Alter positiv mit der Reaktivität des BD korrelierte.

In der Kohorte von Kudielka und Kollegen (2004) wiesen weibliche Probandinnen in den Versuchsgruppen der Kinder und jüngeren Erwachsenen einen signifikant höheren Anstieg der HF auf als männliche Studienteilnehmer. Auch Sato und Miyake (2004), Caroll (2007) und Lovallo (2012) fanden diesen Effekt des Geschlechts auf die KVR. Taylor und Kollegen (2006) konnten in einer älteren Studienkohorte keine Geschlechterunterschiede in der Reaktivität der HF auf den TSST finden, allerdings wiesen weibliche Probanden hier eine verzögerte Recovery der HF auf (die HF zeigte sich nach der Erholungsphase bei Frauen signifikant höher als bei Männern). Zudem konnte diese Arbeitsgruppe einen geringeren Anstieg des dBD und sBD bei Frauen auf den TSST verzeichnen.

Auch hormonelle Aspekte scheinen sich auf die KVR auszuwirken. So unterliegen die Hormone der an der Stressreaktion beteiligten Systeme HHNA und ANS zirkadianen Schwankungen, sodass die Tageszeit einen Einfluss auf die KVR haben könnte (Koch et al. 2017). Bei Frauen zeigen zudem individuelle Schwankungen der Geschlechtshormone einen Effekt: So konnte eine Variation der KVR in den unterschiedlichen Phasen des Menstruationszyklus (Sato und Miyake 2004; Schallmayer und Hughes 2010) sowie auch abhängig vom Prä-/bzw. Postmenopausestatus (Bairey Merz et al. 1998; Hirokawa et al. 2014) nachgewiesen werden. Auch die Einnahme hormoneller Medikamente wie oraler Kontrazeptiva können die KVR beeinflussen (Straznicky et al. 1998; Schallmayer und Hughes 2010). Des Weiteren gibt es eine Reihe anderer Medikamente, welche sich auf kardiovaskuläre Funktionen auswirken und somit auch eine Beeinflussung der KVR mit sich bringen könnten (z. B. Antihypertensiva wie B-Blocker, $\mathrm{Ca}^{2+}$-Antagonisten, $\mathrm{AT}_{1}$-Antagonisten/ACEHemmer; Antiarrhythmika, Antidepressiva, Benzodiazepine, Antiepileptika; de Rooij et al. 2010; Bornschein 2014; Meyer et al. 2016).

In einem von De Rooij und Kollegen (2010) durchgeführten Versuch zur kardiovaskulären Stressreaktion zeigten depressive Patienten mit antihypertensiver Medikation eine reduzierte Reaktivität der HF. Depressive Patienten, welche Antidepressiva bzw. Anxiolytika einnahmen, wiesen eine erniedrigte Reaktivität der HF und des BD auf.

Auch in einem Versuch von Meyer und Mitarbeitern (2016) konnten beim Vergleich der autonomen Stressreaktion von PTBS-Patientinnen mit und ohne herzwirksame Medikation Unterschiede in der Stressreagibilität einiger hämodynamischer Parameter, u. a. dem MAP, dem totalen peripheren Widerstandsindex (engl.: total periphere resistance index, kurz: TPR-I; beschreibt den TPR normiert auf die Körperoberfläche) und dem Herzindex (engl.: cardiac index, kurz: CI; eignet sich zur Beurteilung der Herzleistung) gefunden werden. 
Weitere mögliche Einflussfaktoren auf die KVR stellen der Body-Mass-Index (BMI) und das Rauchen dar, welche zu einer geringeren kardiovaskulären Stressreaktivität führen könnten (Carroll et al. 2007; de Rooij et al. 2010).

Zudem scheinen einige psychosoziale Faktoren wie depressive Erkrankungen (Carroll et al. 2007; de Rooij et al. 2010; Brindle et al. 2013), Kindheitstraumata (Lovallo et al. 2012; Voellmin et al. 2015; Gooding et al. 2016), Angststörungen (Yuenyongchaiwat et al. 2017), Persönlichkeitsfaktoren (z. B. Feindseligkeit; Raikkonen et al. 1999) und das soziale Umfeld (z. B. soziale Unterstützung; Karlin et al. 2003) die KVR zu beeinflussen. Im folgenden Kapitel wird detailliert auf den Einfluss von Depression und Kindheitstrauma auf die KVR eingegangen.

\subsubsection{Depression, Kindheitstrauma und die kardiovaskuläre Stressreaktion}

Kindheitstrauma und Depression stellen mögliche psychosoziale Risikofaktoren in der Pathogenese kardiovaskulärer Erkrankungen dar (Batten et al. 2004; Dong et al. 2004; Chartier et al. 2009; Afifi et al. 2013; Ginty et al. 2013; Spitzer et al. 2013; Kuehl et al. 2016). Als möglicher vermittelnder Mechanismus wird eine autonome Dysbalance vermutet, welche auch mit einer Modifikation des kardiovaskulären Systems einhergeht (von Känel 2011; Spitzer et al. 2013; 2016). Dies könnte in der Folge zu einer gesteigerten kardiovaskulären Stressreaktivität und verzögerten kardiovaskulären Recovery auf akuten psychosozialen Stress führen.

Eine Pionierstudie zur kardiovaskulären Stressreaktivität bei depressiven und traumatisierten Patienten wurde von Heim und Kollegen (2000) durchgeführt. In dieser Studie wurden zur systematischen Aufschlüsselung des Effekts von Depression und Kindheitstrauma auf die kardiovaskuläre Stressreaktivität bei Frauen 49 Probandinnen untersucht, welche in vier Versuchsgruppen eingeteilt wurden: depressive Patientinnen ohne Kindheitstrauma (MDD-), traumatisierte Probandinnen ohne Depression (ACE), traumatisierte Probandinnen mit Depression (MDD+) und eine gesunde Kontrollgruppe (CON). Zur Stressinduktion wurde der TSST durchgeführt und die HF als kardiovaskulärer Marker erfasst. Diese Arbeitsgruppe konnte nachweisen, dass Patientinnen der Gruppe MDD+ im Vergleich zur CON mit einem erhöhten Anstieg der HF auf den TSST reagierten. Bei ,rein' depressiven bzw. traumatisierten Patientinnen konnte lediglich eine Tendenz einer erhöhten KVR festgestellt werden, welche sich jedoch nicht statistisch signifikant zeigte. Die Kombination beider Störungen schien folglich einen größeren Effekt auf die KVR auszuüben als das alleinige Auftreten von Depression bzw. Kindesmissbrauch. 
Darüber hinaus existieren weitere Studien, welche die KVR bei Patienten mit MDD bzw. ACE untersuchen. In einer Metaanalyse von elf Studien fanden Kibler und Ma (2004) eine erhöhte Stressreaktivität der kardiovaskulären Marker HF, sBD und dBD bei depressiven Patienten, wobei dieser Effekt nicht durchgängig statistisch signifikant war. Auch Matthews und Kollegen (2005) konnten einen erhöhten Anstieg der HF und des TPR bei depressiven Probanden nachweisen. Eine Forschungsgruppe von Otte und Mitarbeitern (2005) zeigte bei traumatisierten Probanden eine erhöhte Ausschüttung von MHPG auf einen psychosozialen Stressor, was eine erhöhte Aktivität der SNA widerspiegelt.

Andere Studien fanden keinen Unterschied in der kardiovaskulären Stressreaktivität bei depressiven im Vergleich zu gesunden Probanden (Guinjoan et al. 1995; Taylor et al. 2006) bzw. eine erniedrigte KVR bei Probanden mit ACE bzw. MDD (Carroll et al. 2007; Salomon et al. 2009; de Rooij et al. 2010; Schwerdtfeger und Rosenkaimer 2011; Lovallo et al. 2012; Brindle et al. 2013; Voellmin et al. 2015; Gooding et al. 2016; Yuenyongchaiwat et al. 2017).

\subsection{Zusammenführung der Befunde}

Kardiovaskuläre Erkrankungen stellen eine immense Belastung für die Gesellschaft und das Gesundheitssystem dar (siehe Kapitel 1.1). Neben klassischen Risikofaktoren wie Alter, Rauchen und Adipositas wird mittlerweile psychosozialem Stress eine bedeutende Rolle in der Genese kardiovaskulärer Erkrankungen zugeschrieben. Dabei stellen Kindheitstraumatisierungen und Depressionen potenzielle psychosoziale Risikofaktoren dar, die zudem häufig bei einzelnen Patienten gemeinsam auftreten (siehe Kapitel 1.2.1, 1.2.2 und 1.2.3). Dennoch konnten die vermittelnden Mechanismen zwischen psychosozialen Determinanten und der Entwicklung von Herz-Kreislauferkrankungen noch nicht abschließend geklärt werden (siehe Kapitel 1.2). Neben einem ungünstigen Gesundheitsverhalten werden direkte pathophysiologische Mechanismen diskutiert. Aus psychophysiologischer Sicht wird davon ausgegangen, dass psychosozialer Stress zu einer Sensitivierung der Stresssysteme respektiv zu einer autonomen Dysbalance - führt, aus der wiederum eine veränderte Stressreaktivität und Recovery resultiert (siehe Kapitel 1.2).

Laut McEwens Theorie der Allostase und allostatischen Last führt sowohl eine erhöhte als auch eine prolongierte Stressreaktion langfristig zu negativen Gesundheitsfolgen (siehe Kapitel 1.3.1.3). Zur Induktion psychosozialen Stresses im experimentellen Setting wurden verschiedene psychosoziale Stresstests entwickelt, wobei der TSST als zentrales Paradigma der Stressforschung eine gute Vergleichbarkeit über verschiedene Studien hinweg erlaubt (siehe Kapitel 1.3.3). Einige prospektive Studien konnten bereits den Zusammenhang zwi- 
schen einer erhöhten kardiovaskulären Stressreaktivität sowie einer verzögerten Recovery und der Entwicklung von KVE bzw. der aHTN nachweisen (z. B. Treiber et al. 2003; Matthews et al. 2004; Steptoe und Marmot 2005; Stewart et al. 2006; Chida und Steptoe 2010; Carroll et al. 2012) (siehe Kapitel 1.3.4).

Zudem existieren einige Studien, welche den Einfluss der psychosozialen Determinanten Depression und Kindheitstraumatisierung auf die kardiovaskuläre Stressreaktivität überprüft haben (de Rooij et al. 2010; Lovallo et al. 2012; Brindle et al. 2013; Voellmin et al. 2015; Gooding et al. 2016; Yuenyongchaiwat et al. 2017), wobei sich die Studienergebnisse inhomogen zeigen (siehe Kapitel 1.3.6). Jedoch gab es bisher nur eine Untersuchung einer Arbeitsgruppe um Heim und Kollegen (2000), die zu einer systematischen Aufschlüsselung des Einflusses von Depression und Kindheitstrauma auf die KVR bei Frauen ein komplettes Studiendesign mit den vier Versuchsgruppen MDD+, MDD-, ACE und CON durchgeführt hat. In dieser Studie zeigten Probandinnen mit Depression und frühkindlicher Traumatisierung die höchste KVR auf den TSST. Die Fallzahl dieser Studie war allerdings relativ gering $(\mathrm{n}=49)$, zudem wurde als kardiovaskulärer Parameter ausschließlich die HF gemessen (siehe Kapitel 1.3.6).

Obgleich eine verzögerte kardiovaskuläre Recovery als von der Reaktivität unabhängiger Risikofaktor in der Entstehung kardiovaskulärer Erkrankungen identifiziert werden konnte (Schuler und O’Brien 1997; Steptoe und Marmot 2006), wurde die KVRec bei depressiven und früh traumatisierten Probanden nur in einem kleinen Teil der Studien zur kardiovaskulären Stressreaktion untersucht und bisherige Studienergebnisse liefern ein uneindeutiges Bild (Hamer et al. 2007; Salomon et al. 2009; de Rooij et al. 2010; Schwerdtfeger und Rosenkaimer 2011; Meyer et al. 2016) (siehe Kapitel 1.3.6). Zudem liegen unterschiedliche Operationalisierungen dieses Konstruktes vor (siehe Kapitel 1.3.3). Ein komplettes Studiendesign mit den vier Versuchsgruppen MDD+, MDD-, ACE und CON zur systematischen Untersuchung des Einflusses von Depression und Kindheitstraumatisierung auf die kardiovaskuläre Recovery wurde bisher noch nicht durchgeführt, sodass hier eine Forschungslücke vorliegt. Abbildung 1 zeigt die hier aufgeführten Vorüberlegungen als schematische Zusammenfassung. 


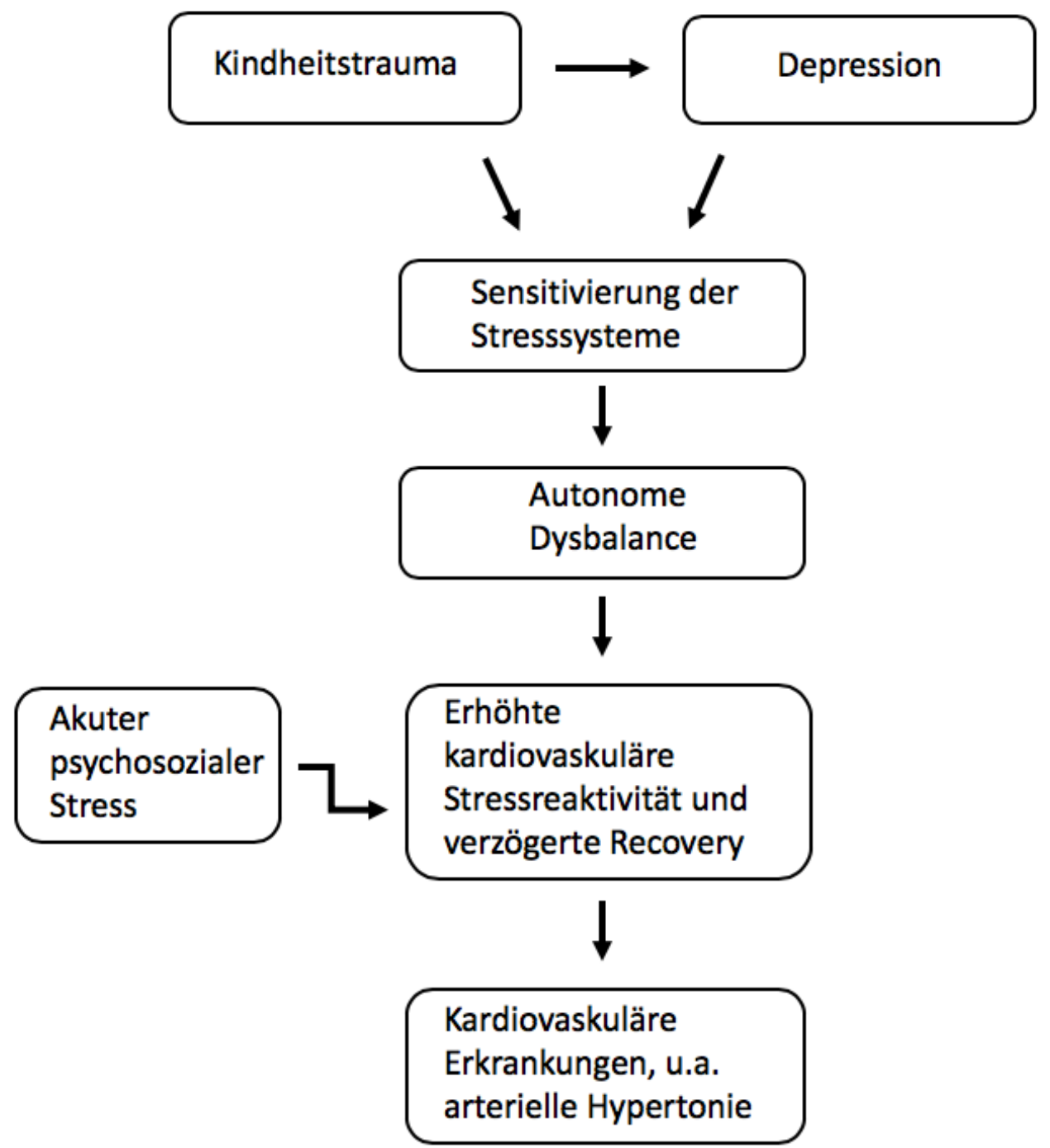

Abbildung 1: Mechanismen, die über eine veränderte kardiovaskuläre Stressreaktion den Zusammenhang zwischen chronisch psychosozialem Stress und der Entstehung kardiovaskulärer Erkrankungen vermitteln könnten

modifiziert nach von Känel (2011) und Spitzer et al. (2013; 2016) 


\subsection{Untersuchungsziel, Fragestellung und Hypothesen}

Ziel der vorliegenden Arbeit ist es, den Forschungsstand hinsichtlich des Einflusses von Depression und/oder Kindheitstraumatisierung auf die kardiovaskuläre Stressreaktivität und Recovery bei Frauen zu ergänzen und somit gleichzeitig zu einer besseren Kenntnis der vermittelnden Mechanismen in der Entwicklung von KVE und der aHTN bei Patienten mit diesen psychosozialen Determinanten beizutragen.

Basierend auf der oben genannten Literatur leitet sich folgende Fragestellung ab, welche die Grundlage dieser Dissertation darstellt:

Welchen Einfluss haben Depression, Kindheitstrauma sowie das gemeinsame Auftreten beider Faktoren auf die kardiovaskuläre Stressreaktivität und Recovery bei Frauen?

Daraus ergeben sich folgende Hypothesen:

1. Hypothese: Patientinnen mit Depression und/oder Kindheitstraumata zeigen im Vergleich zu gesunden Kontrollprobandinnen eine verstärkte kardiovaskuläre Stressreaktivität auf den TSST, welche sich in einem vergleichsweise erhöhten Anstieg der hämodynamischen Parameter HF, sBD und dBD zeigt. Entsprechend der Studienergebnisse von Heim und Kollegen (2000) erwarten wir folgendes Reaktionsmuster: Patientinnen der Versuchsgruppe MDD+ zeigen die höchste Stressreaktivität, gefolgt von den Patientinnen der Gruppen MDD- und ACE. Probandinnen der CON weisen die niedrigste KVR auf.

2. Hypothese: Patientinnen mit Depression und/oder Kindheitstraumata weisen nach dem TSST im Vergleich zu gesunden Kontrollprobandinnen eine verzögerte kardiovaskuläre Recovery auf, welche sich in einer verringerten Rückbildungsfähigkeit der kardiovaskulären Marker HF, sBD und dBD widerspiegelt. Folglich zeigen die Patientinnen der Experimentalgruppen MDD+, MDD- und ACE im Vergleich zur CON

b1. einen geringeren Abfall der kardiovaskulären Parameter nach der Ruhephase im Vergleich zur Stressreaktivität (verzögerte Recovery 1)

b2. eine geringere Wiederannäherung der Messwerte nach der Erholungsphase an die Baseline (verzögerte Recovery 2)

Es wird vermutet, dass Patientinnen der Gruppe MDD+ die geringste KVRec aufweisen, gefolgt von Patientinnen der Gruppe MDD- und ACE. Probandinnen der CON zeigen die größte Erholung der Messparameter. 


\section{Methodik}

Die hier präsentierten Daten stammen aus dem Forschungsprojekt „Stressregulation durch Nahrungsaufnahme? Die Relevanz einer veränderten Regulation der HypothalamusHypophysen-Nebennierenrinden-Achse in Folge lebensgeschichtlich früher Traumatisierungen“. Diese Studie wurde von der Deutschen Forschungsgemeinschaft (DFG) gefördert (DFG Aktenzeichen WI 3396/6-1 und SP 579/3-1) und geht zusammenfassend von folgenden Befunden und Überlegungen aus (Spitzer und Wingenfeld 2012):

Biographisch früher traumatischer Stress, d. h. Traumatisierungen in Kindheit und Jugend, steht in Verbindung mit zahlreichen physiologischen Anpassungsmechanismen, wobei Veränderungen in den Stressregulationssystemen eine besondere Rolle einnehmen. Durch eine verstärkte Aktivität der HHNA bei gleichzeitig verringerter (Feedback-)Sensitivität der Glukocorticoid-Rezeptoren (GR) kommt es konsekutiv zu einer gesteigerten Stressreaktivität. Frühtraumatisierte Personen zeigen ein erhöhtes Risiko zur Entstehung kardiovaskulärer und metabolischer Erkrankungen. Eine mögliche Ursache liegt darin begründet, dass frühtraumatisierte Personen durch eine vermehrte Nahrungsaufnahme in Stresssituationen versuchen, die erhöhte Aktivität der HHNA zu drosseln. Diese Vermutung wird durch vorangegangene Tierstudien gestützt, in welchen es bei chronisch gestressten Tieren durch eine erhöhte Aufnahme von Nahrung zu einer Hemmung der CRH-Ausschüttung und dadurch zu einer Herrunterregulation der HHNA kommt.

Dabei liegt dem Experiment folgendes Modell zugrunde: Biographisch früher Stress führt zu einer veränderten Aktivität der HHNA. Nach Stressexposition versuchen frühtraumatisierte Personen, durch eine vermehrte Nahrungsaufnahme die gestörte Aktivität der HHNA zu normalisieren. Langfristig kommt es dadurch zu metabolischen und kardiovaskulären Folgeerscheinungen wie z. B. Adipositas, einer Insulinresistenz und der aHTN.

Unter Berücksichtigung des Vorliegens einer Depression sollten in dieser Studie traumatisierte Probanden untersucht und die folgenden Hypothesen überprüft werden (Spitzer und Wingenfeld 2012):

1) Patienten mit frühen Traumatisierungen zeigen eine erhöhte Stressreaktivität

2) Zur Stressregulation wird vermehrt Nahrung aufgenommen

3) Die vermehrte Nahrungsaufnahme führt zu metabolischen Veränderungen einschließlich Markern des metabolischen Syndroms

Die in dieser Arbeit dargestellten Ergebnisse beziehen sich auf die erste Hypothese. Ein Aspekt der physiologischen Stressantwort ist die kardiovaskuläre Stressreaktivität (vgl. dazu 
auch die einleitenden Kapitel), die im Rahmen dieser Dissertation im Fokus steht. Hinsichtlich weiterer Details oder anderer Aspekte des Forschungsprojektes wird auf die entsprechende Literatur verwiesen (Wingenfeld et al. 2017a; 2017b; Spitzer et al. 2018).

\subsection{Studiendesign}

Bei der Studie handelt es sich um ein klinisches Forschungsvorhaben an Menschen im Sinne einer experimentellen Querschnittsuntersuchung; es handelt sich ausdrücklich nicht um einen Heilversuch. Die Studie wurde von den zuständigen Ethikkommissionen beraten und positiv beschieden (Votum der Ethikkommission der Universitätsmedizin Göttingen vom 06.05.2013; Antragsnummer 22/1/13).

Die Untersuchung wurde an zwei Zentren, der Klinik für Psychiatrie und Psychotherapie des Asklepios Fachklinikums Tiefenbrunn und der Klinik für Psychiatrie und Psychotherapie der Universitätsmedizin Charité Berlin, Campus Benjamin Franklin, von Dezember 2012 bis Oktober 2015 durchgeführt.

\subsubsection{Aufbau und Ablauf der Studie}

Die eigentlichen Experimente zur Untersuchung der Stressreaktivität mittels TSST fanden in zwei Sitzungen statt, wobei jede Sitzung ca. zwei Stunden umfasste; die beiden Sitzungen wurden in einem zeitlichen Abstand von mindestens zwei Tagen durchgeführt. Im Vorfeld wurden die Probandinnen umfassend klinisch untersucht, um die Ein- und Ausschlusskriterien zu verifizieren (siehe Kapitel 2.2). Dies umfasste ein strukturiertes klinisches Interview für DSM-IV (Saß et al. 2003) zur Erfassung von psychischen Störungen (Achse-IStörungen, z. B. Depressionen, Angst- und Anpassungsstörungen) und Persönlichkeitsstörungen (Achse II-Störungen, z. B. paranoide, schizoide und dependente Persönlichkeitsstörungen). Zur Einschätzung der biographisch frühen Traumatisierungen wurde ein halbstrukturiertes Interview geführt, das ETI (Bremner et al. 2000; Wingenfeld et al. 2011). Die Schwere und das Ausmaß der Depression wurden anhand der MADRS (Montgomery und Åsberg 1979; Williams und Kobak 2008) erfasst. Zudem bearbeiteten die Probandinnen noch weitere Selbstbeurteilungsverfahren zu Depressivität und Kindheitstraumatisierungen: Den CTQ (Bernstein und Fink 1998; Wingenfeld et al. 2010) und das BDI-II (Beck et al. 1996; Hautzinger et al. 2009). Die diagnostischen Interviews und Fragebögen werden detailliert in Abschnitt 2.3 dargestellt.

Die experimentellen Sitzungen begannen an beiden Versuchstagen um jeweils ca. 17 Uhr. Während des Versuchs wurden zu sechs Messzeitpunkten u. a. Blutdruck- und Pulswerte bestimmt. Zu Beginn der Sitzung konnten sich die Probandinnen zunächst für 15 Minuten 
ausruhen, um ihr Stressniveau zu senken. Anschließend wurden in randomisierter Reihenfolge zunächst entweder der TSST (Kirschbaum et al. 1993) oder seine Placebo-Variante, der Placebo-Trier Social Stress Test (P-TSST; Het et al. 2009), durchgeführt. Nach dem TSST bzw. P-TSST füllten die Probandinnen einen Fragebogen zur Erfassung des subjektiv erlebten Stresses aus. Er umfasste neun Items (z. B. persönliche Involvierung in die Aufgabe, Neuheit, Schwierigkeit), wobei sie ihre persönliche Einschätzung auf einer Skala von 1 bis 7 angeben konnten. Danach wurden die Teilnehmerinnen dazu eingeladen, sich an einem Buffet mit Snacks zu bedienen. Dabei wurden sowohl kalorienarme als auch kalorienreiche, süße und salzige Speisen angeboten. Die Probandinnen wussten zu diesem Zeitpunkt nicht, dass die Kalorienaufnahme dokumentiert wurde. Zum Abschluss nahmen sie noch an einer neuropsychologischen Testung teil. Der genaue Ablauf der Studie ist aus Abbildung $2 \mathrm{zu}$ entnehmen.

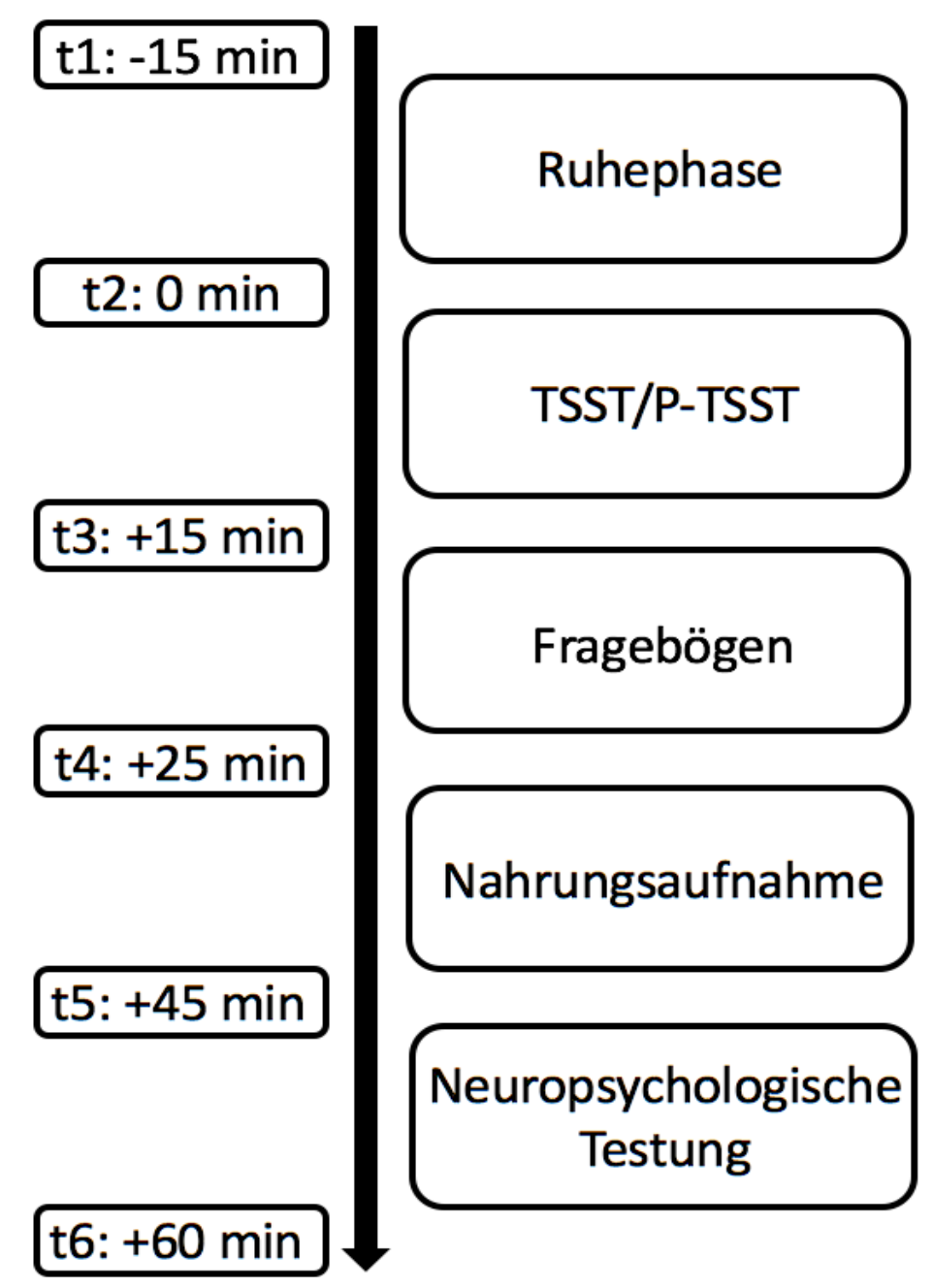

Abbildung 2: Darstellung des Versuchsablaufs 


\subsubsection{Experimentelle Stressinduktion - der Trier Social Stress Test}

In diesem Forschungsvorhaben wurde der Fokus primär auf die autonome Stressreaktion gelegt. Als standardisiertes und unter Laborbedingungen reliabel und valide durchführbares Verfahren zur Induktion von psychosozialem Stress hat sich international der Trier Social Stress Test (Kirschbaum et al. 1993) etabliert, der mittlerweile als zentrales Paradigma in der experimentellen Stressforschung gilt (Birkett 2011; Bali und Jaggi 2015). Um zu überprüfen, ob die hier untersuchten Parameter der Stressreaktivität tatsächlich nur stressspezifisch ansteigen, wurde als Kontrollbedingung der P-TSST (Het et al. 2009) durchgeführt. Dies stellte jedoch nur einen Nebenaspekt des Gesamtforschungsprojekts dar, weshalb in dieser Dissertation nicht näher darauf eingegangen wird. Im Folgenden wird der TSST detailliert dargestellt.

Kirschbaum und Kollegen (1993) entwickelten mit dem TSST eine standardisierte Methode zur experimentellen Stressinduktion. Der TSST setzt sich zusammen aus einer anfänglichen Ruhephase, einer kurzen Präparationsphase und der eigentlichen Testphase, bestehend aus einem simulierten Bewerbungsgespräch und einer Rechenaufgabe. Für unseren Versuch orientierten wir uns an dem normierten Versuchsprotokoll von Kudielka und Mitarbeitern (2007).

Nach Ankunft in unserem Labor konnte die Probandin zunächst 15 Minuten ruhen, um ihr Stresslevel zu reduzieren und somit die Auswirkungen möglicher vorangegangener stressvoller Situationen auf die Untersuchung gering zu halten. Anschließend wurde sie vom Versuchsleiter in einen neuen Raum gebracht, in dem schon ein Gremium, bestehend aus zwei Mitgliedern, wartete. Die Probandin erhielt nun vom Versuchsleiter mündlich standardisierte Instruktionen für die kommende Aufgabe. Laut Anweisungen stellte die Probandin eine Job-Bewerberin dar, die sich zu einem Bewerbungsinterview mit den Personalmanagern trifft. Die Probandin habe fünf Minuten Zeit, um sich auf eine fünfminütige Rede für die Kandidatur um ihren ,Traumjob’ vorzubereiten. Anschließend könne das Komitee noch weitere Fragen stellen. Die Mitglieder des Komitees seien in der Analyse des verbalen und nonverbalen Verhaltens geschult. Die Probandin wurde darüber aufgeklärt, dass ihre Bewerbungsrede per Video- und Tonband aufgezeichnet und die Aufnahmen anschließend durch das Gremium analysiert würden. Die Komiteemitglieder trugen Laborkittel und waren mit Stoppuhren ausgestattet. Sie wurden dazu angewiesen, sich während des gesamten Versuchs der Probandin gegenüber neutral zu verhalten.

Nachdem sich der Untersucher versichert hatte, dass die Probandin die Anleitung verstanden hatte, begann die fünfminütige Vorbereitungszeit. In dieser Zeit verließen die Komi- 
teemitglieder und der Versuchsleiter den Raum. Die Probandin durfte sich schriftliche Notizen machen, welche sie jedoch während der Rede nicht verwenden durfte.

Nach Ablauf der Präparationsphase kam das Komitee wieder in den Versuchsraum, Kamera und Tonbandgerät wurden eingeschaltet. Die Probandin hatte nun fünf Minuten Zeit, um ihre Rede zu halten. Sobald sie diese innerhalb von drei Minuten unterbrach, wurde sie dazu aufgefordert, fortzufahren. Falls sie nach Ablauf der drei Minuten pausierte, schwieg das Gremium mindestens 15 Sekunden lang und begann dann, standardisierte Fragen zu stellen (z. B. „Was halten Sie von Teamarbeit?“, „Welche Führungsqualitäten besitzen Sie?““ und „Was halten Sie von Freunden?“). Sobald die fünf Minuten abgelaufen waren, wurde die Probandin vom Komitee unterbrochen.

An die Rede schloss sich eine fünfminütige Rechenaufgabe an, wobei die Probandin so exakt und so schnell wie möglich von 2083 in Dreizehner-Schritten subtrahieren sollte. Bei jedem Rechenfehler wurde die Probandin dazu aufgefordert, wieder bei 2083 zu beginnen. Bei sehr starken Rechenschwierigkeiten wurde die Aufgabe vereinfacht, sodass in SiebenerSchritten von 2083 subtrahiert werden sollte.

Sobald die Testphase abgeschlossen war, wurde die Probandin über das Ziel der Studie aufgeklärt und es wurde ihr versichert, dass keine Video- und Tonbandaufnahmen stattgefunden hatten. Es wurde Wert auf ein positives Feedback gelegt. Bei diesem Nachgespräch war immer mindestens eine der Personen, die ein ,Komiteemitglied' dargestellt hatte, anwesend.

Blutdruck und Puls wurden innerhalb dieses Ablaufs zu definierten Zeitpunkten gemessen (s. 2.1.1, Seite 20):

- t1: Nach Ankunft der Probandin (-15 min)

- t2: nach der Ruhephase, direkt vor dem TSST (0 min)

- t3: direkt nach dem TSST (+15 min)

- t4: nach der Erholungsphase (+25 min)

Der TSST beinhaltet einige Merkmale, die nachweislich eine Stimulation der HHNA fördern, wie soziale Bewertung, Einbeziehung des Selbstwerts und Unkontrollierbarkeit der Situation. Es konnte zudem nachgewiesen werden, dass die Kombination einer verbalinteraktiven Aufgabe (Rede) und einer kognitiven Aufgabe (Rechnen) dieses Stresssystem am stärksten aktiviert (Dickerson und Kemeny 2004). Dadurch kommt es zu einem signifikanten Anstieg von ACTH, Cortisol (in Serum und Speichel), GH und Prolaktin (Kirschbaum et al. 1993).

Auch das SNS reagiert infolge des TSST mit einer erhöhten Ausschüttung von Adrenalin und Noradrenalin (Gold et al. 2004). Es kommt während des TSST zuverlässig zu einer 
Erhöhung kardiovaskulärer Parameter (Kirschbaum et al. 1993; Jezova et al. 2004; Jönsson et al. 2010).

\subsection{Studienteilnehmer}

Da sich bei der kardiovaskulären Stressreaktivität als auch bei Parametern kardiovaskulärer Erkrankungen Geschlechtsunterschiede finden (Kudielka et al. 2004; Sato und Miyake 2004; Taylor et al. 2006; Ladwig et al. 2013; Robert Koch-Institut 2015), wurden ausschließlich weibliche Studienteilnehmer rekrutiert.

Um die Effekte von Depressivität bzw. einer majoren Depression und von Kindheitstraumatisierungen auf die kardiovaskuläre Stressreaktion differenziert analysieren zu können, wurden die Probandinnen in vier Untersuchungsgruppen eingeteilt, welche in Tabelle 1 synoptisch dargestellt sind.

Es wurde versucht, diese vier Untersuchungsgruppen hinsichtlich Alter, Bildung und Nikotinkonsum zu parallelisieren. Die Rekrutierung erfolgte über öffentliche Aushänge und Anzeigen; zusätzlich wurden Patientinnen mit der Diagnose einer MDD über die spezialisierten Stationen der an dieser Studie beteiligten psychiatrisch-psychotherapeutischen Kliniken gewonnen. Nach gründlicher Überprüfung der Ein- und Ausschlusskriterien wurden Probandinnen, die einer der vier Untersuchungsgruppen zugeordnet werden konnten, ausführlich und schriftlich über die Studie aufgeklärt. Nur wenn eine schriftliche Einverständniserklärung vorlag, erfolgte die Aufnahme in die Studie. Probandinnen, die extern, d. h. nicht direkt über die beteiligten Kliniken rekrutiert wurden, erhielten für ihre Teilnahme eine Aufwandsentschädigung in Höhe von $200 €$.

Tabelle 1: Übersicht über die vier Versuchsgruppen

\begin{tabular}{|l|cccc|}
\hline & MDD+ & MDD- & ACE & CON \\
& $(\mathbf{n}=\mathbf{3 2})$ & $(\mathbf{n}=\mathbf{5 2})$ & $\mathbf{( n = 2 2 )}$ & (n= 37) \\
\hline Charakterisierung & Probandinnen mit & Probandinnen mit & Gesunde Proban- & Gesunde Pro- \\
der Versuchs- & Depression und & Depression ohne & dinnen mit & bandinnen \\
gruppen & frühen Traumati- & frühe Traumati- & frühen Traumati- & \\
& sierungen & sierungen & sierungen & \\
& &
\end{tabular}

\subsubsection{Allgemeine Ein- und Ausschlusskriterien}

Es wurden nur volljährige ( $\geq 18$ Jahre) und einwilligungsfähige Probandinnen eingeschlossen. In allen Versuchsgruppen führten folgende somatische und psychische Kriterien zur Exklusion: ZNS-relevante somatische Erkrankungen, Schädel-Hirn-Traumata in der Vor- 
geschichte, schwerwiegende somatische Erkrankungen wie z. B. maligne Tumoren, HIVInfektion, höhergradige Herzrhythmusstörungen sowie Herzinfarkte, klinisch manifester Bluthochdruck, Stoffwechselerkrankungen wie Diabetes mellitus, organisch bedingte Veränderung der Cortisolsekretion, Einnahme oraler und inhalativer Glucocorticoide, Autoimmunkrankheiten, aktuelle und/oder chronische Infektionen, Schwangerschaft und ein BMI über 30 (d. h. ab Adipositas Stadium I). Ausgeschlossen wurden außerdem Probandinnen mit einer manifesten PTBS. Weil bei Patientinnen mit einer PTBS eine gestörte Stressreaktivität unumstritten ist (Zaba et al. 2015; Meyer et al. 2016), könnte es bei deren Einschluss in diese Studie zu einer erheblichen Verzerrung der Ergebnisse kommen und damit die hier interessierende Fragestellung nicht beantwortet werden können. Zudem führte das Vorliegen von psychotischen Störungen, von Abhängigkeitserkrankungen und Anorexia nervosa zum Studienausschluss. Andere subsyndromal bestehende oder leichte Achse-I-Störungen (z. B. eine leichte Panikstörung) stellten keinen Ausschlussgrund dar. Probandinnen, deren Depression sich aktuell in Remission befand, wurden nicht für die Studie berücksichtigt, da sie nicht eindeutig einer Versuchsgruppe zugeteilt werden konnten. Nicht teilnehmen konnten außerdem Probandinnen, die innerhalb der letzten Woche psychotrope Medikamente wie Neuroleptika, Benzodiazepine oder Opiate eingenommen hatten.

\subsubsection{Spezifische Ein- und Ausschlusskriterien}

Die Diagnose einer majoren Depression wurde mittels eines SKID-Interviews (siehe Kapitel 2.3.1.1) überprüft, sodass die Probandinnen auf dieser Grundlage den dargestellten Gruppen zugeordnet werden konnten.

Das Merkmal einer lebensgeschichtlich frühen Traumatisierung wurde in Anlehnung an die Publikation der Arbeitsgruppe um Heim (2000) definiert als wiederholter (mindestens einmal pro Monat über mindestens ein Jahr), moderater bis schwerer sexueller oder körperlicher Missbrauch, gekennzeichnet durch erzwungene Berührung der Geschlechtsorgane einer erwachsener Person, intime Berührung der eigenen Geschlechtsorgane durch eine andere Person, versuchter oder vollzogener vaginaler, oraler und/oder analer Geschlechtsverkehr, Schläge, Stöße, Würgen, Bedrohung oder Verletzung mit einer Waffe sowie das Einsperren und Fesseln vor dem Alter von 18 Jahren. Die Überprüfung dieser Kriterien erfolgte mit einem klinischen Interview, der deutschen Übersetzung des ETI (siehe Kapitel 2.3.1.2), in dem diese Ereignisse erfasst werden. Zudem werden auch ,weichere' Formen früher Traumatisierungen erfasst. Mit Hilfe der Befunde aus dem ETI erfolgte die Zuordnung zu den Untersuchungsgruppen mit versus ohne frühe Traumatisierung. 
Für Probandinnen ohne frühe Traumatisierungen wurde zudem gefordert, dass sie keinen gravierenden Belastungen in der Kindheit und Jugend ausgesetzt waren; dazu zählten auch langfristige Trennung von den Eltern, Vernachlässigung, Tod oder Scheidung der Eltern, Adoption, schwere Unfälle und Erkrankungen sowie Naturkatastrophen.

\subsection{Instrumente}

In diesem Unterkapitel werden die verwendeten Instrumente, die zur Erhebung unserer Daten verwendet wurden, dargestellt.

\subsubsection{Diagnostische Interviews und Fragebögen}

Zur Verifizierung unserer Ein- und Ausschlusskriterien kamen verschiedene psychometrische Instrumente zum Einsatz. Psychische Störungen wurden anhand des strukturierten klinischen Interviews für DSM-IV (SKID; Fydrich et al. 1997) erfasst. Zudem wurden zum Screening auf eine frühkindliche Traumatisierung und eine bestehende Depression weitere diagnostische Interviews und Fragebögen angewandt. Im Folgenden werden diese Verfahren detailliert erläutert.

\subsubsection{Strukturiertes klinisches Interview für DSM-IV}

Das SKID ist ein semistrukturiertes Interview zur Erfassung, Diagnostik und Klassifizierung psychischer Störungen, wie sie in dem Diagnosemanual DSM-IV auf den Achsen I (,Klinische Störungen') und II (,Persönlichkeitsstörungen') operationalisiert sind. Dieses in den USA entwickelte Verfahren ist mittlerweile international sehr verbreitet und in Deutschland seit 1997 verfügbar (Wittchen et al. 1997). Das SKID ist für den Einsatz bei erwachsenen Probanden mit (vermuteten) psychischen Erkrankungen geeignet und setzt sich aus folgenden Materialien zusammen:

- Handanweisung für SKID-I und SKID-II

- Interviewheft SKID-I inklusive Beurteilungsheft

- Interviewheft SKID-II inklusive Auswertungsbogen

- Fragebogen SKID-II

Mithilfe des SKID-I werden psychische Störungen auf Achse I des DSM-IV erfasst. Das Interview ist dabei in verschiedene Sektionen eingeteilt, die folgende Störungen abdecken: affektive und psychotische Störungen, Störungen durch psychotrope Substanzen, Angsterkrankungen, somatoforme Störungen, Essstörungen und Anpassungsstörungen. In jeder Sektion werden die jeweiligen Symptome der entsprechenden Achse I-Störung in den letzten vier Wochen erfasst (derzeitige Episode), aber auch Angaben zum zeitlichen Verlauf 
der Beschwerden und deren Schweregrad kodiert, so dass am Ende des Interviews eine Beurteilung zu Art, Schweregrad und Verlaufsform der Störungen möglich wird, ebenso eine Einschätzung zur aktuellen sowie zur Lebenszeitdiagnose.

Das SKID-II ermöglicht in einem zweistufigen Vorgehen die Diagnostik der zehn auf Achse II sowie der zwei im Anhang des DSM-IV aufgeführten Persönlichkeitsstörungen. In einem ersten Schritt werden über den SKID-II Fragebogen als Selbstbeurteilungsverfahren die einzelnen Merkmale der Persönlichkeitsstörungen erfragt. Im sich anschließenden Interviewteil des SKID-II werden alle Kriterien, die im Fragebogen bejaht wurden, näher untersucht und kodiert.

Die Durchführungsdauer des SKID-I beträgt zwischen 80 und 100 Minuten, das SKID-IIInterview etwa 30 Minuten. Hinsichtlich der psychometrischen Gütekriterien kann nach entsprechenden Schulungen von Durchführungs- und Auswertungsobjektivität ausgegangen werden. Das Verfahren wurde in verschiedenen Studien ausführlich mit guten psychometrischen Befunden bezüglich Anwendbarkeit, Reliabilität und Effizienz untersucht (Maffei et al. 1997; Zanarini und Frankenburg 2001; Lobbestael et al. 2011).

\subsubsection{Early Trauma Inventory}

Das ETI (Bremner et al. 2000) ist ein Inventar zur retrospektiven Erfassung psychotraumatischer Ereignisse in Kindheit und Jugend (bis zum 18. Lebensjahr). Anhand von 55 Items werden folgende vier Untergruppen von Traumata unterschieden: erzieherische/körperliche Bestrafung (EKB; 9 Items), emotionale (EE; 8 Items) und sexuelle Erfahrungen (SE; 15 Items) und generelle Traumata (GT; 24 Items, z. B. Todesfälle, Naturkatastrophen, Scheidung/Trennung der Eltern).

Der Interviewer stellt geschlossene Fragen zu jeder Domäne (z. B. zur Erfassung des physischen Missbrauchs: „Wurden Sie jemals mit der Hand geschlagen?`). Dabei werden neben dem Auftreten (ja/nein) auch die Dauer (Alter bei Anfang bis Alter bei Ende des Ereignisses) und die Häufigkeit $(0=$ nie bis $6=>1 /$ Tag $)$ differenziert nach der Altersstufe $(0$ 5, 6-12, 13-18 Jahre) erfasst. Zudem werden die Probanden nach der Auswirkung des Ereignisses auf ihr Leben befragt $(-3=$ sehr negativ, $0=$ keine Auswirkung, $+3=$ sehr positiv).

In der Auswertung des ETI können Schweregrad-Indizes für die einzelnen Domänen und ein Gesamtwert (GES; 0-62 Punkte) berechnet werden. Dazu werden für alle positiv beantworteten Items Häufigkeit und Dauer multipliziert und die Ergebnisse aufsummiert.

Wingenfeld und Kollegen (2011) konnten zufriedenstellende psychometrische Kennwerte für die deutsche Version des ETI nachweisen. 


\subsubsection{Childhood Trauma Questionnaire}

Als retrospektives Selbstbeurteilungsverfahren von Vernachlässigung und Traumata in der Kindheit und Jugend (bis zum 18. Lebensjahr) wurde in unserem Forschungsprojekt der CTQ angewendet. Die ursprüngliche amerikanische Langversion des Verfahrens wurde von Bernstein und Kollegen entwickelt (1994). Diese Arbeitsgruppe veröffentlichte zudem eine gekürzte englischsprachige Version des Fragebogens, welche mittlerweile international verbreitet ist (Bernstein und Fink 1998). Wingenfeld und Kollegen (2010) sowie eine Arbeitsgruppe um Klinitzke (2012) konnten die gute psychometrische Qualität für die deutschsprachige Fassung des CTQ bestätigen.

Der CTQ wird in der Diagnostik bei Patienten ab zwölf Jahren angewandt. Es handelt sich um einen mehrdimensionalen Fragebogen bestehend aus einer Eingangsinstruktion für den Probanden und 28 Items zur Selbstbeurteilung. In Anbetracht der sehr persönlichen Fragen wird der Proband in der Einleitung zunächst dazu ermutigt, so ehrlich wie möglich zu antworten. Der CTQ erfasst fünf Dimensionen von Kindesmisshandlungen, welche sich in aktive Formen - sexueller (SM), körperlicher (KM) und emotionaler Missbrauch (EM) sowie passive Formen - emotionale (EV) und körperliche Vernachlässigung (KV) - einteilen lassen. Alle Items werden mit der Formulierung „Als ich aufgewachsen bin...“ eingeleitet und anschließend mit Aussagen über Missbrauch/Vernachlässigung weitergeführt, z. B. „...wwrde ich von Familienmitgliedern als dumm, faul oder hässlich bezeichnet“ und „...hatte ich eine perfekte Kindheit“. Die Probanden können ihre Erfahrungen zu den Angaben auf einer fünfstufigen Likert-Skala ( 1 = überhaupt nicht; $5=$ sehr häufig) beurteilen. Jede Skala (z. B. sexueller Missbrauch) wird in fünf Items abgefragt, wobei pro Skala minimal fünf Punkte (keine Misshandlung in Kindheit und Jugend) bis maximal 25 Punkte (extreme Misshandlung) erreicht werden können. In die Auswertung fließen außerdem drei Items einer Minimierungs- und Verleugnungsskala ein, welche eine Tendenz zur Verharmlosung und sozial erwünschtes Antwortverhalten aufdecken soll. Für die Auswertung kann der Schweregrad der Misshandlung für die einzelnen Subskalen in vier Stufen eingeteilt werden: kein oder minimaler, moderater, schwerer oder schwerer bis extremer Missbrauch. In der Regel sind fünf bis zehn Minuten für die Bearbeitung des CTQ ausreichend (Bernstein und Fink 1998).

\subsubsection{Beck Depressions-Inventar}

Das BDI (Beck et al. 1961) ist ein international anerkanntes psychometrisches Verfahren zum Screening auf eine möglicherweise bestehende Depression und zur Selbsteinschätzung des Schweregrads derselben. Die hier verwendete deutschsprachige Version BDI-II (Beck 
et al. 1996; Hautzinger et al. 2009) orientiert sich an den Depressionskriterien des DSM-IV (Saß et al. 2003). Der Fragebogen besteht aus 21 Items, welche depressive Symptome wie z. B. Traurigkeit, Pessimismus oder Versagensgefühle abdecken. Bei 19 der 21 Items soll der Patient auf einer vierstufigen Likert-Skala von 0 bis 3 das Zutreffen der Aussagen bezogen auf die letzten zwei Wochen einschätzen $(0=$ nicht vorhanden; $3=$ starke Ausprägung). Einzig die beiden Items, Veränderung der Schlafgewohnheit' und ,Veränderung des Essverhaltens' heben sich ab. Um sowohl die Zu- als auch die Abnahme von Appetit und Schlaf genau zu erfassen, hat der Proband hier sieben Antwortmöglichkeiten zur Verfügung, wobei sich die erste Antwortmöglichkeit auf das Nichtzutreffen der Aussage (z. B. „Ich habe meine Schlafgewohnheiten nicht verändert“), die übrigen sechs auf die Veränderung der Gewohnheit beziehen („Ich schlafe mehr als sonst“ bis zu „Ich wache ein bis zwei Stunden früher auf als gewöhnlich und kann dann nicht mehr einschlafen“).

Anhand der Angaben kann ein Summenscore berechnet werden, welcher den Grad der aktuellen Depressivität ausdrückt. Es können maximal 63 Punkte erreicht werden. Durch vorgegebene Schwellenwerte kann das Ergebnis in eine der folgenden fünf Unterkategorien eingeordnet werden: keine Depression (0-8 Punkte), minimale Depression (9-13 Punkte), leichte Depression (14-19 Punkte), mittelschwere Depression (20-28 Punkte), schwere Depression (29-63 Punkte). Die Durchführungsdauer des BDI-II beträgt ungefähr fünf bis zehn Minuten. In mehreren internationalen Studien konnten für den BDI-II gute psychometrische Kennwerte (hohe Reliabilität, sowie Konstrukt- und inhaltliche Validität) gezeigt werden (Beck et al. 1996; Krefetz et al. 2002; Hautzinger et al. 2009).

\subsubsection{Montgomery-Åsberg Depression Rating Scale}

Die MADRS (Montgomery \& Åsberg 1979, Williams \& Kobak 2008 ) ist ein Verfahren zur Fremdeinschätzung der Ausprägung einer bestehenden Depression. Diese wird anhand von zehn für depressive Patienten symptomtypischen Items (z. B. sichtbare Traurigkeit, innere Spannungen und verminderter Appetit) gemessen. Bei der Beantwortung der Fragen soll sich der Proband auf sein Befinden innerhalb der vergangenen Woche beziehen. Die Beurteilung stützt sich auf das Interview und die Beobachtungen durch den Untersucher, welcher die eingeschätzte Ausprägung jedes Items auf einer siebenstufigen Likert-Skala von 0-6 angeben kann $(0=$ keine/kaum Symptome; $6=$ schwere Ausprägung der Symptomatik). Die Aufsummierung der einzelnen Werte ergibt die Gesamtsumme, welche die Schwere der Depression widerspiegelt. Muller und Kollegen (2000) schlagen für die Interpretation des Gesamtscores folgende Einteilung anhand von Grenzwerten vor: keine Depression (0-8 Punkte), milde Depression (9-17 Punkte), moderate Depression (18-34 Punkte), 
schwere Depression (35-60 Punkte). Die inhaltliche Reliabilität und Validität gelten für die deutsche Version der MADRS als gesichert (Maier und Philipp 1985; Schmidtke et al. 1988).

\subsubsection{Erfassung physiologischer Parameter}

\subsubsection{Messung von Blutdruck und Puls}

Zur Messung von Blutdruck und Puls wurde ein vollautomatisches Blutdruckmessgerät für den Oberarm (boso medicus uno; boso, Deutschland) angewandt (siehe Abbildung 3). Es besteht aus einer Blutdruckmanschette (Cuff) und einem digitalen Messgerät. Zunächst wird die Manschette am Oberarm angebracht. Nach Messstart wird sie über eine elektrische Pumpe so lange aufgepumpt, bis der Druck im Cuff den systolischen Blutdruck übersteigt und somit kein Blut mehr durch die Arteria brachialis fließen kann. Durch ein elektrisches Ventil wird die Luft nun langsam aus dem Cuff gelassen. Sensoren erfassen dabei den aktuellen Blutdruck sowie die sich ändernden Blutflussgeräusche. Sobald der systolische Blutdruck den Manschettendruck wieder übersteigt, kann in der Systole Blut in die Arteria brachialis fließen und die Sensoren erfassen zu diesem Zeitpunkt den systolischen Blutdruckwert. In der Diastole sinkt der Blutdruck wieder und solange der Manschettendruck den diastolischen Druck noch übersteigt, kommt es zu Verwirbelungen im Blut und Strömungsgeräuschen. Ist der Druck im Cuff soweit gesunken, dass der diastolische Wert ihn übersteigt, sind keine Strömungsgeräusche mehr messbar. Der Druck, welcher beim letzten Strömungsgeräusch messbar ist, entspricht dem diastolischen Blutdruck. Die Sensoren führen gleichzeitig eine Messung der Herzfrequenz durch. Die Blutdruckmanschette wurde den Patienten für jede Messung neu angelegt.

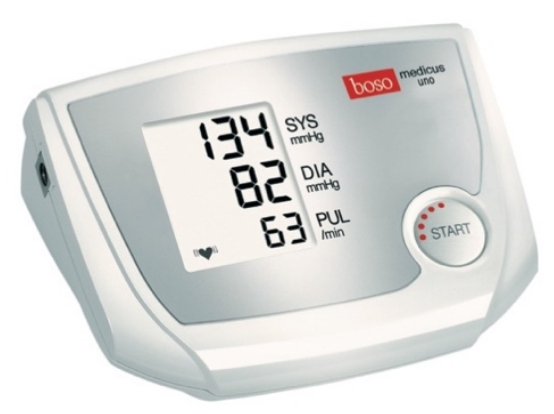

Abbildung 3: Blutdruckemessgerät "boso medicus uno" (mit freundlicher Genehmigung der BOSCH + SOHN GmbH u. Co. KG) 


\subsection{Datenverarbeitung und statistische Auswertung}

Die in unserem Versuch erhobenen patientenbezogenen Daten wurden anonymisiert. Die statistische Auswertung und das Erstellen von Abbildungen und Graphiken erfolgte mit Microsoft Excel (2016) und der Datenanalyse-Software Statistica (Version 12).

Im folgenden Abschnitt werden detaillierte Informationen zu Datensatz, Operationalisierung und statistischer Auswertung gegeben.

\subsubsection{Datensatz}

Ein Datensatz mit soziodemographischen, klinischen sowie versuchsbezogenen Daten bildete die Grundlage dieser Dissertation und wurde zur Überprüfung der Hypothesen ausgewertet.

Folgende Variablen des Datensatzes wurden statistisch aufgearbeitet:

- Soziodemographische und klinische Daten

○ Alter

○ Größe

○ Gewicht

- Schuljahre

- Schulabschluss

- Familienstand

○ Rauchen

- Nebendiagnosen

- Somatische Medikamente

○ Psychopharmaka

- Ergebnisse der psychometrischen Tests

- Blutdruck- und Pulswerte zu den verschiedenen Messzeitpunkten innerhalb des Versuchs

\subsubsection{Operationalisierung der Stressreaktivität und Recovery}

Zur Operationalisierung unserer Hypothesen legten wir Folgendes fest: Die gemessenen kardiovaskulären Parameter bei t 2 definierten wir als Baseline, da die Patienten im Vorfeld eine Viertelstunde lang eine ruhige Aktivität ausgeübt hatten und das angenommene Stresslevel hier am geringsten war. Die KVR operationalisierten wir in Anlehnung an andere Untersuchungen (de Rooij et al. 2010; Lovallo et al. 2012; Brindle et al. 2013; Voellmin et al. 2015; Gooding et al. 2016) als den Anstieg der Parameter von t2 (Baseline) nach t3 (nach dem TSST). Weil das Konstrukt der KVRec in der Literatur nicht einheitlich definiert ist (siehe Kapitel 1.3.3), legten wir zwei Operationalisierungen zugrunde:

- Recovery 1 beschreibt die Veränderung der Parameter von t3 (nach dem TSST) zu t4 (nach der Erholungsphase). Dadurch soll überprüft werden, ob die kardiovaskulären Parameter nach der Erholungsphase ( $\mathrm{t} 4$ ) wieder abgesunken waren. Mit der 
Recovery 1 wird die Erholung der Parameter in Abhängigkeit von der Stressreaktivität betrachtet.

- Recovery 2 beschreibt die Veränderung der kardiovaskulären Parameter nach der Erholungsholungsphase ( $\mathrm{t} 4)$ in Abhängigkeit zur Baseline ( $\mathrm{t} 2)$ und ist unabhängig von der Stressreaktivität. Sie ist definiert als das Verhältnis der Blutdruck- und Pulswerte von Baseline (t2) und Erholungsphase (t4).

Eine schematische Darstellung der Operationalisierung der KVR und KVRec wird in den Abbildungen 4 und 5 gegeben.

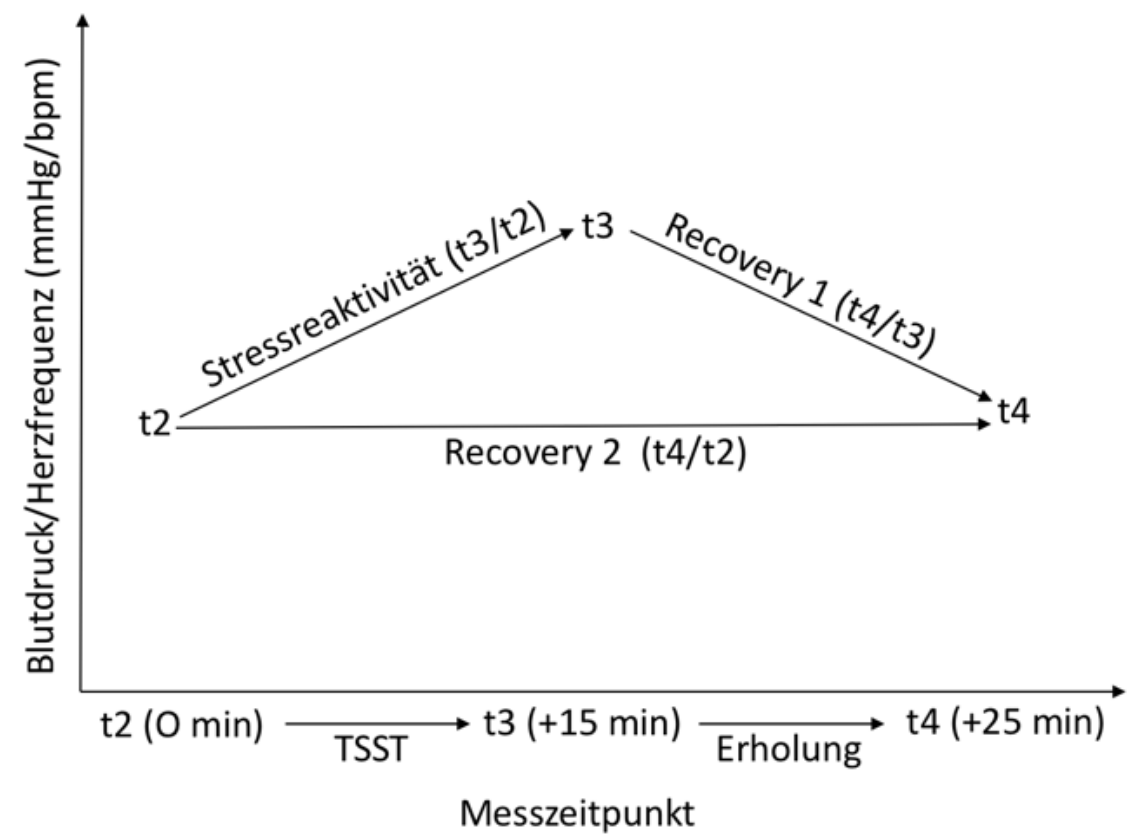

Abbildung 4: Operationalisierung der Stressreaktivität und Recovery 


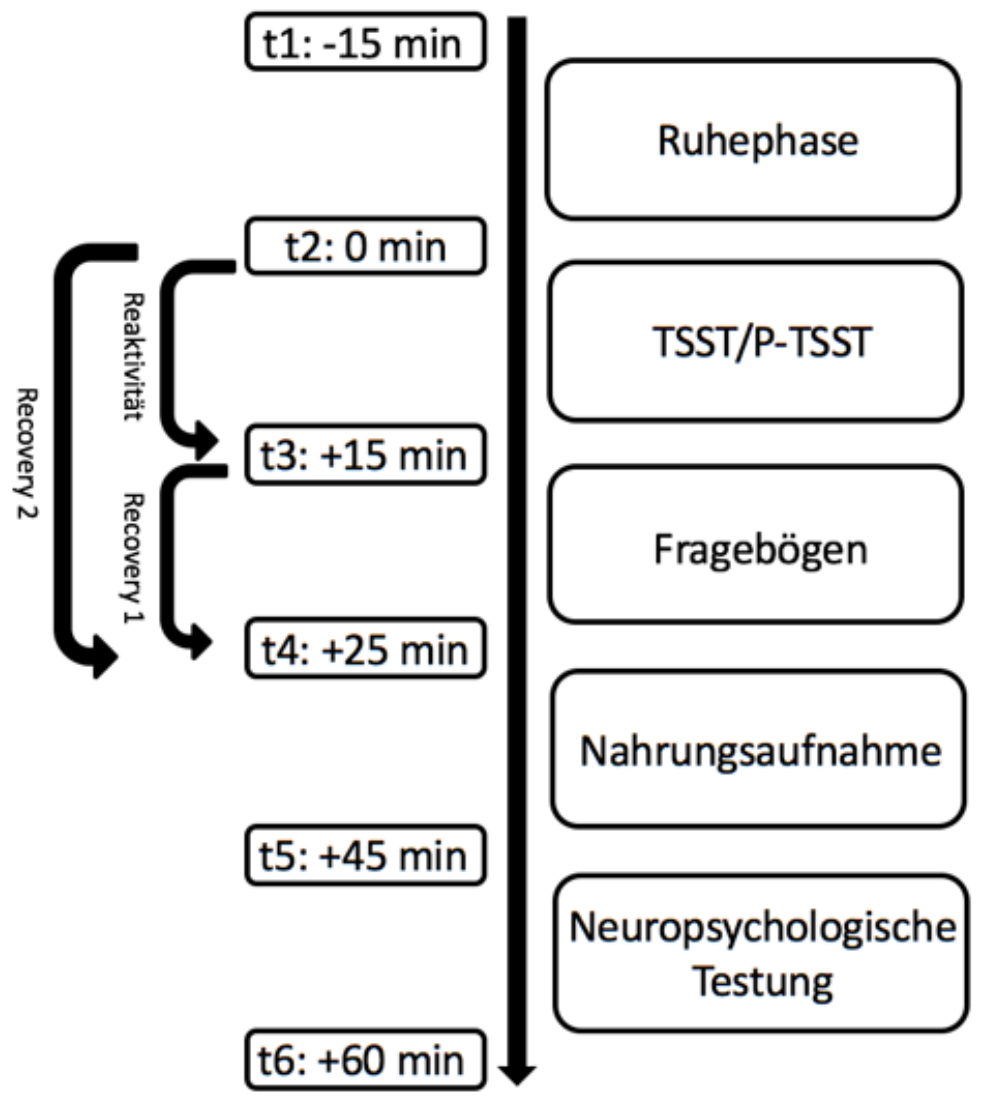

Abbildung 5: Versuchsablauf, Stressreaktivität und Recovery

\subsubsection{Statistische Auswertung}

In diesem Unterkapitel werden die verwendeten statistischen Verfahren näher erläutert.

Für den $\alpha$-Fehler wurde ein Signifikanzniveau von $5 \%(0,05)$ festgelegt, wobei ein p-Wert von $\leq .05$ einem signifikanten, $\leq .01$ einem sehr signifikanten und $\leq .001$ einem hoch signifikanten Ergebnis entspricht.

\subsubsection{Statistische Auswertung der soziodemographischen und klinischen Daten}

Zur Klassifikation der soziodemographischen und klinischen Merkmale ergaben sich verschiedene Skalierungen. Kontinuierliche Daten auf dem Niveau einer Verhältnisskala (Alter, Körpergröße, Gewicht, BMI, Anzahl der Schuljahre) sind als Mittelwerte (M) \pm Standardabweichung (Standard deviration; SD) angegeben. Zunächst wurden für diese Parameter Quantile-Quantile-Plots (Q-Q-Plots) angefertigt. Dieses Verfahren diente der graphischen Überprüfung der Normalverteilung der abhängigen Variable innerhalb der Studienkohorte vor der Durchführung weiterer parametrischer Testverfahren. Da eine Normalver- 
teilung gegeben war, wurden zum Vergleich der Verteilung dieser Merkmale zwischen den vier Versuchsgruppen einfaktorielle Varianzanalysen (ANOVA) durchgeführt.

Kategoriale Merkmale auf Nominalskalenniveau (Schulabschluss, Familienstand, Rauchen, Nebendiagnosen, somatische Medikamente, Psychopharmaka) wurden als absolute Häufigkeiten (n) und Prozente (\%) angegeben. Die Testung der Signifikanz erfolgte bei diesen Daten per Pearson $\chi^{2}$-Test.

\subsubsection{Statistische Auswertung der psychometrischen Tests}

Die Ergebnisse der psychometrischen Tests wurden ordinalskaliert. Es wurden Mittelwerte und Standardabweichungen für die einzelnen Versuchsgruppen berechnet. Zur weiteren statistischen Auswertung wurden die Daten zunächst mithilfe von Q-Q-Plots auf Normalverteilung getestet. Da keine Normalverteilung gegeben war, wurde zur Überprüfung der Gruppenunterschiede der Kruskal-Wallis-Test angewendet. Dieses nichtparametrische Verfahren eignet sich zum Vergleich von mehr als zwei unverbundenen Stichproben, die nicht normalverteilt sind. Ein signifikantes Ergebnis drückt jedoch nur aus, dass sich die Erwartungswerte global unterscheiden, zeigt allerdings nicht, wo die Unterschiede genau liegen. Um Auskunft darüber zu erhalten, welche Versuchsgruppen sich in ihren Testergebnissen signifikant voneinander unterscheiden, wurde daher anschließend als Post-hocTest ein Mann-Whitney-U-Test durchgeführt. In diesem statistischen Verfahren werden für jeweils zwei Versuchsgruppen paarweise Mittelwertvergleiche durchgeführt. Da beim multiplen Testen die Wahrscheinlichkeit für einen $\alpha$-Fehler stark ansteigt, wurde zur Neutralisierung eine Bonferroni-Korrektur angewandt. Zur übersichtlichen Darstellung der Lagemaße (Quartile, Maxima, Minima, Mediane) und der Gruppenunterschiede wurden BoxWhisker-Plots angefertigt.

\subsubsection{Statistische Auswertung des TSST und Überprüfung der Hypothesen}

Um einen Eindruck über die Veränderung der kardiovaskulären Paramater während des TSST zu erhalten, wurden für die einzelnen Versuchsgruppen Verlaufsgraphiken erstellt.

In Anlehnung an einen vergleichbaren Versuch von Gooding und Kollegen (2016) wurden Quotienten der Messzeitpunkte gebildet und in Prozente umgewandelt. Die Stressreaktivität und Recovery werden somit als prozentualer Anstieg bzw. Abfall der Messparameter angegeben:

- $(\mathrm{t} 3 / \mathrm{t} 2-1) \cdot 100 \cong$ Stressreaktivität $(\%)$

- $(\mathrm{t} 4 / \mathrm{t} 3-1) \cdot 100 \cong$ Recovery $1(\%)$

- $\quad(\mathrm{t} 4 / \mathrm{t} 2-1) \cdot 100 \cong$ Recovery $2(\%)$ 
Anschließend wurden Mittelwerte und Standardabweichungen für Blutdruck- und Pulswerte der Baseline, Stressreaktivität, Recovery 1 und Recovery 2 des Gesamtkollektivs und der einzelnen Versuchsgruppen bestimmt. Für die weitere statistische Bearbeitung wurde die Normalverteilung mittels Q-Q-Plots ermittelt, welche bestätigt werden konnte. Um zu überprüfen, ob die mittleren prozentualen Anstiege bzw. Abfälle der kardiovaskulären Parameter für die einzelnen Versuchsgruppen signifikant waren, wurden Einstichproben-tTests gegen 0 sowie eine Adjustierung des $\alpha$-Niveaus mittels Bonferroni-Holm vorgenommen. Zur Überprüfung der Hypothesen wurden Gruppenvergleiche mittels einfaktorieller Varianzanalysen durchgeführt. Da die Patientinnen bezüglich der Verteilung ihrer soziodemographischen Daten keine statistischen Unterschiede aufwiesen, wurden diese Einflussgrößen als nicht-verzerrende Störgrößen betrachtet (Weiß 2013) und in der statistischen Aufarbeitung der Versuchsergebnisse nicht weiter berücksichtigt. Zur Darstellung der Lagemaße der Stressreaktivität, Recovery 1 und Recovery 2 für die einzelnen kardiovaskulären Parameter wurden Box-Whisker-Plots erstellt. Da eine große Standardabweichung auffiel, wurde die Verteilung der prozentualen Veränderungen der kardiovaskulären Parameter in den positiven bzw. negativen Bereich deskriptiv in Prozent pro Versuchsgruppe aufgeschlüsselt. 


\section{Ergebnisse}

\subsection{Stichprobenbeschreibung}

\subsubsection{Soziodemographische Daten}

An der hier vorliegenden Studie nahmen insgesamt 143 Probandinnen teil. Die Teilnehmerinnen wurden anhand der bereits oben dargestellten Kriterien den Versuchsgruppen MDD+, MDD-, ACE und CON zugeteilt. Es wurde versucht, die vier Versuchsgruppen bezüglich ihrer soziodemographischen Merkmale zu parallelisieren (siehe Kapitel 2.2). In der Auswertung zeigte sich, dass die vier Versuchsgruppen bezüglich der soziodemographischen Daten keine signifikanten Unterschiede aufwiesen.

Die Probandinnen waren zum Zeitpunkt der Messung im Mittel 34,9 \pm 11,6 Jahre alt $(\mathrm{F}=0,46 ; \mathrm{p}=.71)$. Die durchschnittliche Größe betrug $167 \pm 6,6 \mathrm{~cm}(\mathrm{~F}=1,38 ; \mathrm{p}=.25)$ und das Gewicht $64,4 \pm 11,7 \mathrm{Kg}(\mathrm{F}=0,59 ; \mathrm{p}=.63)$. Der mittlere BMI lag bei 22,8 $\pm 3,4$ $\mathrm{Kg} / \mathrm{m}^{2}(\mathrm{~F}=1,79 ; \mathrm{p}=.15)$.

Bezüglich der Schulbildung ist Folgendes festzuhalten: Die vier Versuchsgruppen wiesen in der mittleren Anzahl der Schuljahre keine statistischen Unterschiede auf, wobei die hier untersuchten Probandinnen durchschnittlich 11,6 $\pm 1,4$ Schuljahre absolviert hatten $(\mathrm{F}=2,02 ; \mathrm{p}=.11)$. Bei der Betrachtung der Schulabschlüsse hatten 62,7\% der Befragten einen Gymnasial-, 30,1 \% einen Realschul- und 5,6 \% einen Hauptschulabschluss absolviert. Lediglich eine Probandin $(0,7 \%)$ besaß zum Zeitpunkt der Untersuchung keinen Schulabschluss. Hier zeigte sich eine statistische Tendenz zu Gruppenunterschieden, wobei die Probandinnen der CON die höchsten Schulabschlüsse aufwiesen $(F=16,42 ; \mathrm{p}=.06)$. Die Probandinnen der Gruppe ACE befanden sich in dieser Studienkohorte hinsichtlich des Schulabschlusses im Mittelfeld, gefolgt von den Versuchsteilnehmerinnen der Versuchsgruppen mit einer führend depressiven Erkrankung (MDD+ und MDD-), welche die niedrigsten Schulabschlüsse auswiesen.

Bezüglich des Familienstands zeigte sich, dass der größte Teil der Probandinnen ledig war $(68,4 \%$ ), jeweils $15,8 \%$ waren verheiratet bzw. getrennt oder geschieden $(F=8,08$; $\mathrm{p}=.23)$.

Knapp mehr als ein Drittel (39,1\%) der Probandinnen rauchte $(\mathrm{F}=1,58 ; \mathrm{p}=.66)$. 
Eine detaillierte Darstellung der aufgeführten deskriptiven Daten der einzelnen Versuchsgruppen sowie die ermittelten F-, $\chi^{2}$ - und p-Werte sind in Tabelle 2 einzusehen.

Tabelle 2: Soziodemographische Merkmale

\begin{tabular}{|c|c|c|c|c|c|c|c|}
\hline & $\begin{array}{c}\text { Gesamt } \\
(n=143)\end{array}$ & $\begin{array}{l}\text { MDD+ } \\
(n=32)\end{array}$ & $\begin{array}{l}\text { MDD- } \\
(\mathrm{n}=52)\end{array}$ & $\begin{array}{c}\text { ACE } \\
(n=22)\end{array}$ & $\begin{array}{c}\text { CON } \\
(n=37)\end{array}$ & $\begin{array}{c}\text { F- } \\
\text { Wert }\end{array}$ & $\begin{array}{c}\mathrm{p}- \\
\text { Wert }\end{array}$ \\
\hline $\begin{array}{l}\text { Alter } \\
\text { (in Jahren)* }\end{array}$ & $\begin{array}{c}34,9 \\
(11,6)^{1}\end{array}$ & $\begin{array}{c}34,2 \\
(10,5)\end{array}$ & $\begin{array}{c}35,5 \\
(11,7)\end{array}$ & $\begin{array}{c}36,5 \\
(12,2)\end{array}$ & $\begin{array}{c}33,2 \\
(11,9)^{2}\end{array}$ & 0,46 & .71 \\
\hline $\begin{array}{l}\text { Größe } \\
\text { (in m)* }\end{array}$ & $\begin{array}{l}1,67 \\
(0,1)\end{array}$ & $\begin{array}{l}1,67 \\
(0,1)\end{array}$ & $\begin{array}{l}1,68 \\
(0,1)\end{array}$ & $\begin{array}{l}1,65 \\
(0,1)\end{array}$ & $\begin{array}{l}1,69 \\
(0,1)\end{array}$ & 1,38 & .25 \\
\hline $\begin{array}{l}\text { Gewicht } \\
\text { (in Kg)* }\end{array}$ & $\begin{array}{c}64,4 \\
(11,7)\end{array}$ & $\begin{array}{c}63,8 \\
(10,9)\end{array}$ & $\begin{array}{c}65 \\
(12,1)\end{array}$ & $\begin{array}{c}66,4 \\
(12,1)\end{array}$ & $\begin{array}{l}62,7 \\
(9,4)\end{array}$ & 0,59 & .63 \\
\hline $\begin{array}{l}\text { BMI } \\
\left(\text { in } \mathrm{Kg} / \mathrm{m}^{2}\right)^{*}\end{array}$ & $\begin{array}{c}22,8 \\
(3,54)\end{array}$ & $\begin{array}{l}22,7 \\
(3,1)\end{array}$ & $\begin{array}{l}23 \\
(4)\end{array}$ & $\begin{array}{l}24,1 \\
(3,6)\end{array}$ & $\begin{array}{c}22 \\
(3,1)\end{array}$ & 1,79 & .15 \\
\hline Schuljahre* & $\begin{array}{c}11,6 \\
(1,4)^{3}\end{array}$ & $\begin{array}{c}11,1 \\
(1,5)^{4}\end{array}$ & $\begin{array}{c}11,6 \\
(1,4)^{5}\end{array}$ & $\begin{array}{c}11,6 \\
(1,4)^{6}\end{array}$ & $\begin{array}{c}12 \\
(1,2)^{7}\end{array}$ & 2,02 & .11 \\
\hline & Gesamt & MDD+ & MDD- & ACE & $\mathrm{CON}$ & $\begin{array}{c}\chi^{2}- \\
\text { Wert }\end{array}$ & $\begin{array}{c}\text { p- } \\
\text { Wert }\end{array}$ \\
\hline $\begin{array}{l}\text { Schul- } \\
\text { abschluss }^{\#}\end{array}$ & $\frac{142}{(100 \%)}$ & $\underline{32}$ & $\underline{51}$ & $\begin{array}{c}\underline{22} \\
(100 \%)\end{array}$ & $\underline{37}$ & & \\
\hline Abitur & $\begin{array}{c}89 \\
(62,7 \%)\end{array}$ & $\begin{array}{c}16 \\
(50 \%)\end{array}$ & $\begin{array}{c}29 \\
(56,8 \%)\end{array}$ & $\begin{array}{c}15 \\
(68,2 \%)\end{array}$ & $\begin{array}{c}29 \\
(78,4 \%)\end{array}$ & & \\
\hline Realschule & $\begin{array}{c}44 \\
(30,1 \%)\end{array}$ & $\begin{array}{c}10 \\
(32,3 \%)\end{array}$ & $\begin{array}{c}20 \\
(39,2 \%)\end{array}$ & $\begin{array}{c}6 \\
(27,3 \%)\end{array}$ & $\begin{array}{c}8 \\
(21,6 \%)\end{array}$ & 16,42 & .06 \\
\hline Hauptschule & $\begin{array}{c}8 \\
(5,6 \%)\end{array}$ & $\begin{array}{c}5 \\
(15,6 \%)\end{array}$ & $\begin{array}{c}2 \\
(3,9 \%)\end{array}$ & $\begin{array}{c}1 \\
(4,5 \%)\end{array}$ & $\begin{array}{c}0 \\
(0 \%)\end{array}$ & & \\
\hline keinen & $\begin{array}{c}1 \\
(0,7 \%) \\
\end{array}$ & $\begin{array}{c}1 \\
(3,1 \%) \\
\end{array}$ & $\begin{array}{c}0 \\
(0 \%) \\
\end{array}$ & $\begin{array}{c}0 \\
(0 \%) \\
\end{array}$ & $\begin{array}{c}0 \\
(0 \%) \\
\end{array}$ & & \\
\hline $\begin{array}{l}\text { Familien- } \\
\text { stand }^{\#}\end{array}$ & $\frac{139}{(100 \%)}$ & $\underline{32}$ & $\frac{51}{(100 \%)}$ & $\begin{array}{c}21 \\
(100 \%) \\
\end{array}$ & $\frac{35}{(100 \%)}$ & & \\
\hline$\overline{\text { ledig }}$ & $\begin{array}{c}95 \\
(68,4 \%)\end{array}$ & $\begin{array}{c}22 \\
(68,8 \%)\end{array}$ & $\begin{array}{c}35 \\
(68,6 \%)\end{array}$ & $\begin{array}{c}16 \\
(76,2 \%)\end{array}$ & $\begin{array}{c}22 \\
(62,9 \%)\end{array}$ & & \\
\hline verheiratet & $\begin{array}{c}22 \\
(15,8 \%)\end{array}$ & $\begin{array}{c}3 \\
(9,4 \%)\end{array}$ & $\begin{array}{c}12 \\
(23,5 \%)\end{array}$ & $\begin{array}{c}1 \\
(4,8 \%)\end{array}$ & $\begin{array}{c}6 \\
(17,1 \%)\end{array}$ & 8,08 & .23 \\
\hline getrennt & $\begin{array}{c}22 \\
(15,8 \%)\end{array}$ & $\begin{array}{c}7 \\
(21,9 \%) \\
\end{array}$ & $\begin{array}{c}4 \\
(7,8 \%)\end{array}$ & $\begin{array}{c}4 \\
(19 \%) \\
\end{array}$ & $\begin{array}{c}7 \\
(20 \%) \\
\end{array}$ & & \\
\hline$\underline{\text { Rauchen }}^{\#}$ & $\underline{143}$ & $\begin{array}{c}32 \\
(100 \%) \\
\end{array}$ & $\begin{array}{c}52 \\
(100 \%) \\
\end{array}$ & $\begin{array}{c}22 \\
(100 \%) \\
\end{array}$ & $\begin{array}{c}37 \\
(100 \%) \\
\end{array}$ & & \\
\hline $\mathrm{Ja}$ & $\begin{array}{c}56 \\
(39,1 \%)\end{array}$ & $\begin{array}{c}15 \\
(46,9 \%)\end{array}$ & $\begin{array}{c}21 \\
(40,4 \%)\end{array}$ & $\begin{array}{c}7 \\
(31,8 \%)\end{array}$ & $\begin{array}{c}13 \\
(35,1 \%)\end{array}$ & 1,58 & .66 \\
\hline
\end{tabular}

* Ergebnisse sind präsentiert als Mittelwerte und Standardabweichung einschließlich F- und p-Werte der ANOVA. \# Ergebnisse sind präsentiert als absolute Häufigkeiten und Prozente einschließlich $\chi^{2}$ - und $p$-Werte des $\chi^{2}$-Tests. MDD+: Patientinnen mit frübkindlicher Traumatisierung und Depression, MDD-: depressive Patientinnen obne frübkindliche Traumatisierung, ACE:gesunde Probandinnen mit frübkindlicher Traumatisierung, CON: Kontrollgruppe. ${ }^{1} n=142,{ }^{2} n=36,{ }^{3} n=132,{ }^{4} n=30,{ }^{5} n=50,{ }^{6} n=20,{ }^{7} n=32$ 


\subsubsection{Begleitdiagnosen}

Anhand des SKID I- und II-Interviews wurden die Probandinnen auf mögliche komorbide psychische Störungen untersucht. Es fiel auf, dass depressive Patientinnen oftmals weitere psychiatrische Diagnosen aufwiesen. In der Gruppe MDD+ zeigten 40,7 \% der Patientinnen mindestens eine psychiatrische Nebendiagnose, in der Gruppe MDD- waren es 42,3 \%. Diese beiden Versuchsgruppen unterschieden sich nicht bezüglich der Anzahl der Nebendiagnosen pro Probandin $\left(\chi^{2}=2,65 ; p=.62\right)$. In der Versuchsgruppe ACE wies lediglich eine Probandin eine psychische Erkrankung in Form einer leichten Panikstörung auf, welche jedoch laut unseren allgemeinen Ein- und Ausschlusskriterien (siehe Kapitel 2.2.1) keinen Grund zur Exklusion darstellte. Die Probandinnen der CON zeigten definitionsgemäß keine psychischen Störungen. Eine genaue Übersicht über die Anzahl der Begleitdiagnosen pro Probandin differenziert nach Versuchsgruppen sowie über die ermittelten $\chi^{2}$ - und p-Werte ist in Tabelle 3 dargestellt. Abbildung 6 gibt einen Überblick über die Art der Nebendiagnosen nach ICD-10 (ICD-10-GM 2019) sowie über die Anzahl der festgestellten Nebendiagnosen pro Versuchsgruppe.

Tabelle 3: Anzabl der ermittelten Begleitdiagnosen pro Probandin der Versuchsgruppen mit einer führend depressiven Erkerankung

\begin{tabular}{|c|c|c|c|c|}
\hline $\begin{array}{l}\text { Begleitdiagnosen } \\
\text { pro Probandin }\end{array}$ & $\begin{array}{l}\text { MDD+ } \\
(n=32)\end{array}$ & $\begin{array}{l}\text { MDD- } \\
(\mathrm{n}=52)\end{array}$ & $\chi^{2}$-Wert & p-Wert \\
\hline $\mathbf{0}$ & $\begin{array}{c}19 \\
(59,4 \%)\end{array}$ & $\begin{array}{c}30 \\
(57,7 \%)\end{array}$ & & \\
\hline 1 & $\begin{array}{c}10 \\
(31,3 \%)\end{array}$ & $\begin{array}{c}22 \\
(42,3 \%)\end{array}$ & & \\
\hline 2 & $\begin{array}{c}2 \\
(6,3 \%)\end{array}$ & $\begin{array}{c}0 \\
(0 \%)\end{array}$ & 2,65 & .62 \\
\hline 3 & $\begin{array}{c}1 \\
(3,1 \%)\end{array}$ & $\begin{array}{c}0 \\
(0 \%)\end{array}$ & & \\
\hline
\end{tabular}

Ergebnisse sind präsentiert als absolute Häufigkeiten und Prozente einschließlich $\chi^{2}$ und $p$-Werte des $\chi^{2}$-Tests. MDD+: Patientinnen mit frübkindlicher Traumatisierung und Depression, MDD-: depressive Patientinnen obne frübkindliche Traumatisierung 


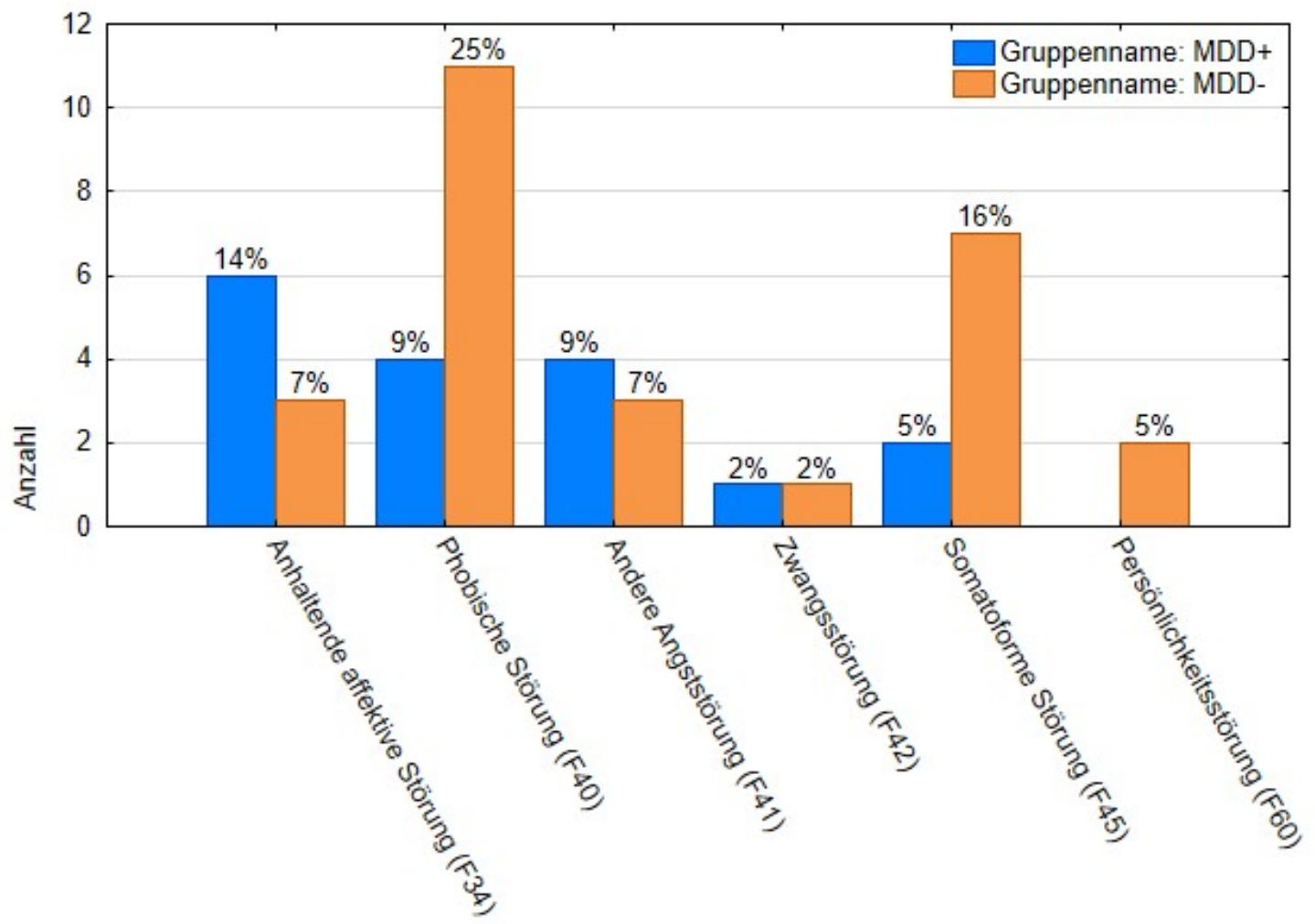

Komorbide Diagnose

Abbildung 6: Übersicht über die ermittelten Nebendiagnosen für die Versuchsgruppen mit einer führend depressiven Erkrankung

MDD+: Patientinnen mit frübkindlicher Traumatisierung und Depression, MDD-: depressive Patientinnen obne frübkindliche Traumatisierung

\subsubsection{Medikation}

\subsubsection{Somatische Medikamente}

Unsere Studienkohorte war nicht komplett unmediziert. Ungefähr ein Drittel (30,5\%) der Probandinnen nahm ein orales Kontrazeptivum ein $\left(\chi^{2}=1,74 ; p=.63\right)$. Insgesamt neun Probandinnen (6,3\%) bekamen das Schilddrüsenhormon L-Thyroxin $\left(\chi^{2}=2,2 ; \mathrm{p}=.53\right)$. Bezüglich der Einnahme dieser Medikamente gab es keinen signifikanten Unterschied zwischen den Gruppen. Eine Patientin der Gruppe MDD- erhielt einen COX-2-Inhibitor (Coxib) als Dauermedikament. Zudem nahmen zwei Patientinnen der Versuchsgruppe MDD- regelmäßig Betablocker ein, wobei es sich bei der einen Probandin um Bisoprolol, bei der anderen um Metoprolol handelte. Eine genaue Übersicht über die eingenommenen somatischen Medikamente wird in Tabelle 4 gegeben. 
Tabelle 4: Übersicht der eingenommenen somatischen Medikamente

\begin{tabular}{|l|ccccccc|}
\hline & $\begin{array}{c}\text { Gesamt } \\
\mathbf{n = 1 4 3}\end{array}$ & $\begin{array}{c}\text { MDD+ } \\
\mathbf{n = 3 2}\end{array}$ & $\begin{array}{c}\text { MDD- } \\
\mathbf{n = 5 2}\end{array}$ & $\begin{array}{c}\text { ACE } \\
\mathbf{n = 2 2}\end{array}$ & $\begin{array}{c}\text { CON } \\
\mathbf{n}=\mathbf{3 7}\end{array}$ & $\begin{array}{c}\boldsymbol{\chi}^{\mathbf{2}} \text { - } \\
\text { Wert }\end{array}$ & $\begin{array}{c}\text { p- } \\
\text { Wert }\end{array}$ \\
\hline Orales & 43 & 8 & 19 & 6 & 10 & 1,74 & .63 \\
Kontrazeptivum & $(30,5 \%)$ & $(25,8 \%)^{1}$ & $(37,3 \%)^{2}$ & $(27,3 \%)$ & $(27 \%)$ & & \\
\hline L-Thyroxin & 9 & 3 & 3 & 0 & 3 & 2,22 & .53 \\
& $(6,3 \%)$ & $(9,4 \%)$ & $(5,8 \%)$ & $(0 \%)$ & $(8,1 \%)$ & & \\
\hline Betablocker & 2 & 0 & 2 & 0 & 0 & - & - \\
& $(1,4 \%)$ & $(0 \%)$ & $(3,8 \%)$ & $(0 \%)$ & $(0 \%)$ & & \\
\hline Coxib & 1 & 0 & 1 & 0 & 0 & - & - \\
& $(1,43 \%)$ & $(0 \%)$ & $(5,2 \%)$ & $(0 \%)$ & $(0 \%)$ & & \\
\hline
\end{tabular}

Ergebnisse sind präsentiert als absolute Häufigkeiten und Prozente einscbließlich $\chi^{2}$ - und $p$-Werte des $\chi^{2}$-Tests. MDD+: Patientinnen mit frübkindlicher Traumatisierung und Depression, MDD-: depressive Patientinnen obne frübkindliche Traumatisierung, ACE: gesunde Probandinnen mit frühkindlicher Traumatisierung, CON: Kontrollgruppe. ${ }^{1} n=32$, ${ }^{2} n=51$

\subsubsection{Psychopharmaka}

Erwartungsgemäß nahmen nur Patientinnen der Gruppen MDD+ und MDD- Psychopharmaka ein. Die beiden Gruppen unterschieden sich nicht bezüglich der Anzahl der Probandinnen, die mindestens ein Psychopharmakon einnahmen $\left(\chi^{2}=1,84 ; p=.54\right)$. Aus der Versuchsgruppe MDD+ erhielten zwölf Teilnehmerinnen mindestens ein Psychopharmakon (37,5\%), aus der Gruppe MDD- waren es 23 Personen (54,3 \%). Eine genaue Übersicht zeigt Tabelle 5. In Abbildung 7 wird eine graphische Übersicht über die eingenommenen Substanzklassen in den einzelnen Versuchsgruppen gegeben.

Tabelle 5: Anzabl der eingenommenen Psychopharmaka für die Versuchsgruppen mit einer führend depressiven Erkrankung

\begin{tabular}{|c|ccc|c|}
\hline $\begin{array}{c}\text { Psychopharmaka } \\
\text { pro Person }\end{array}$ & $\begin{array}{c}\text { MDD+ } \\
(\mathbf{n}=\mathbf{3 2})\end{array}$ & $\begin{array}{c}\text { MDD- } \\
(\mathbf{n}=\mathbf{5 2})\end{array}$ & $\chi^{\mathbf{2} \text {-Wert }}$ & p-Wert \\
\hline $\mathbf{0}$ & $\begin{array}{c}20 \\
(62,5 \%)\end{array}$ & $\begin{array}{c}29 \\
(55,7 \%)\end{array}$ & & \\
\hline $\mathbf{1}$ & $\begin{array}{c}8 \\
(25 \%)\end{array}$ & $\begin{array}{c}18 \\
(34,6 \%)\end{array}$ & & \\
\hline $\mathbf{2}$ & $\begin{array}{c}4 \\
(12,5 \%)\end{array}$ & $\begin{array}{c}4 \\
(7,7 \%)\end{array}$ & 1,84 & $\mathrm{p}=.54$ \\
\hline $\mathbf{3}$ & $\begin{array}{c}1 \\
(0 \%)\end{array}$ & $(1,9 \%)$ & & \\
\hline
\end{tabular}

Ergebnisse sind präsentiert als absolute Häufigkeiten und Prozente einschließlich $\chi^{2}$ - und $p$-Werte des $\chi^{2}$-Tests. MDD+: Patientinnen mit frühkindlicher Traumatisierung und Depression, MDD-: depressive Patientinnen obne frühkindliche Traumatisierung 


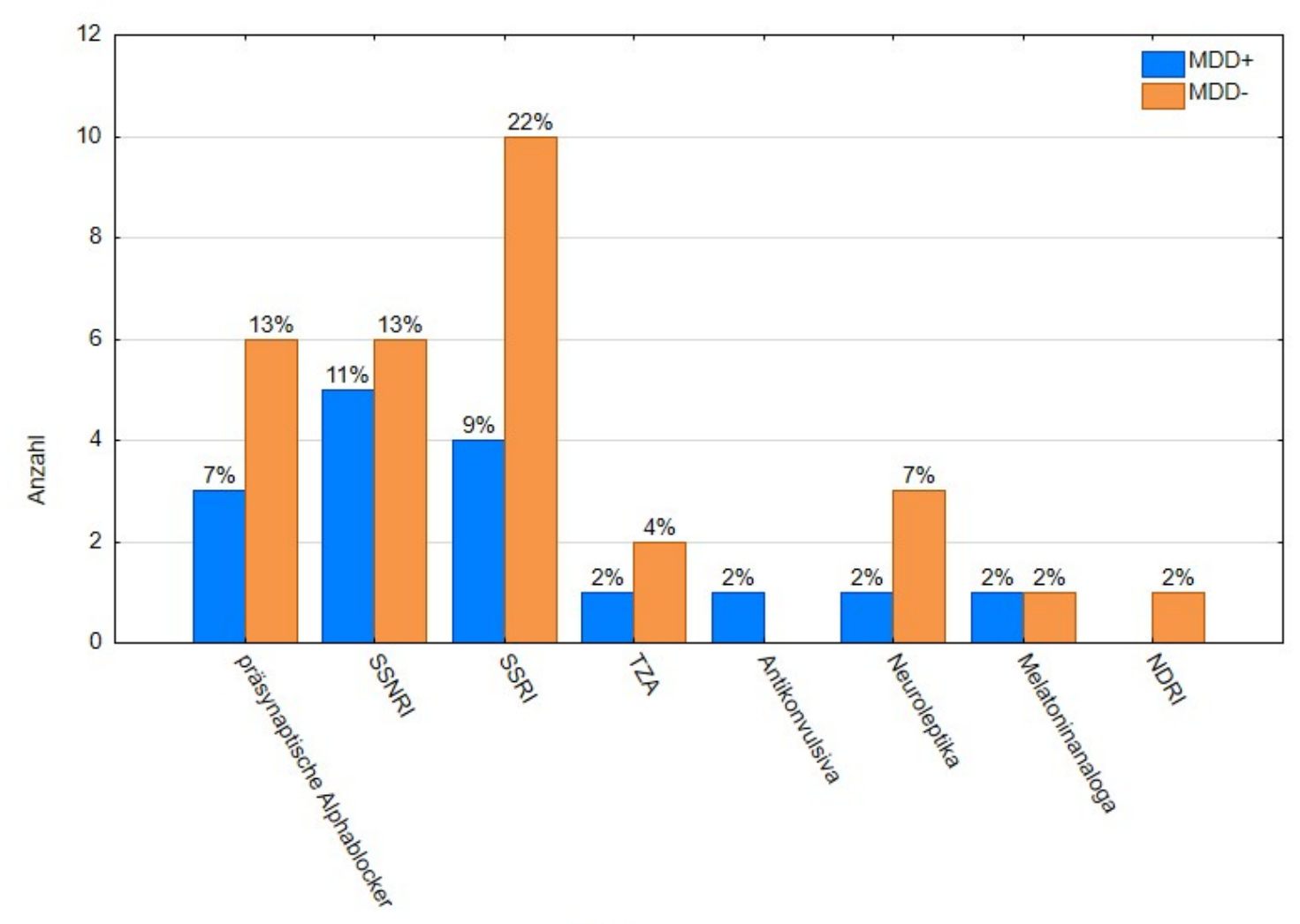

Substanzgruppe

Abbildung 7: Übersicht über eingenommene psychopharmakologische Substanzgruppen für die Versuchsgruppen mit einer führend depressiven Erkrankung

MDD+: Patientinnen mit frübkindlicher Traumatisierung und Depression, MDD-: depressive Patientinnen obne frübkindliche Traumatisierung. SSNRI: Serotonin-Noradrenalin-Wiederaufnabmehemmer, SSRI: Selektive Serotonin-

Wiederaufnabmehemmer, TZA: triaylklisches Antidepressivum, NDRI: Norepinephrin-Dopamin-Wiederaufnabmehemmer

\subsubsection{Frühkindliche Traumatisierung und Depressivität}

\subsubsection{Frühkindliche Traumatisierungen}

Bei der Auswertung der beiden Instrumente zur Erfassung frühkindlicher Traumatisierungen, dem ETI und dem CTQ, zeigte sich folgendes Ergebnis: Wie erwartet wiesen die Versuchsgruppen mit Traumatisierungen, MDD+ und ACE, in den Summenscores der Verfahren und in allen Subskalen die höchsten Werte auf. Die Gruppe MDD- zeigte in den Summenscores und fast allen Unterkategorien signifikant geringere Werte als die Gruppen MDD+ und ACE. Die Kontrollgruppe erreichte in allen Kategorien die geringsten Werte, wobei diese Werte teilweise signifikant niedriger als die der Gruppe MDD- waren. Eine genaue Übersicht der Werte liefert Tabelle 6. In Abbildung 8 sind die Ergebnisse als BoxWhisker-Plot dargestellt. 
Tabelle 6: Ergebnisse des ETI und CTQ differenziert nach Versuchsgruppe

\begin{tabular}{|c|c|c|c|c|c|c|}
\hline ETI & $\begin{array}{l}\text { MDD+ } \\
(n=32)\end{array}$ & $\begin{array}{l}\text { MDD- } \\
(\mathrm{n}=52)\end{array}$ & $\begin{array}{c}\text { ACE } \\
(n=22)\end{array}$ & $\begin{array}{c}\text { CON } \\
(n=37)\end{array}$ & H- Wert & $\begin{array}{c}\mathrm{p}-\text { Wert } \\
\text { (Alle } \mathrm{p}<.01 \text { ) }\end{array}$ \\
\hline GES & $\begin{array}{c}599 \\
(430,4)\end{array}$ & $\begin{array}{c}279,7 \\
(298,6)\end{array}$ & $\begin{array}{c}708,5 \\
(323,3)\end{array}$ & $\begin{array}{c}56,9 \\
(122)\end{array}$ & 80,52 & $\mathrm{a}$ \\
\hline GT & $\begin{array}{c}108 \\
(128) \\
\end{array}$ & $\begin{array}{c}54,2 \\
(62,8) \\
\end{array}$ & $\begin{array}{l}122,6 \\
(84,6) \\
\end{array}$ & $\begin{array}{c}18,8 \\
(39,7)\end{array}$ & 39,22 & $\mathrm{~b}$ \\
\hline EKB & $\begin{array}{c}111,6 \\
(145,3)\end{array}$ & $\begin{array}{c}38,3 \\
(123,6)\end{array}$ & $\begin{array}{c}180,6 \\
(115,6)\end{array}$ & $\begin{array}{c}7,6 \\
(16,6)\end{array}$ & 69,32 & $\mathrm{c}$ \\
\hline $\mathbf{E E}$ & $\begin{array}{c}324 \\
(194)\end{array}$ & $\begin{array}{c}180 \\
(180,6)\end{array}$ & $\begin{array}{c}355,2 \\
(210,1)\end{array}$ & $\begin{array}{c}28,9 \\
(99,7)\end{array}$ & 66,94 & $\mathrm{a}$ \\
\hline SE & $\begin{array}{c}55,3 \\
(89,6)\end{array}$ & $\begin{array}{c}7,2 \\
(19,7)\end{array}$ & $\begin{array}{c}50 \\
(62,1)\end{array}$ & $\begin{array}{c}1,6 \\
(5,1)\end{array}$ & 46,32 & $\mathrm{c}$ \\
\hline CTQ & $\begin{array}{l}\text { MDD+ } \\
(\mathrm{n}=30)\end{array}$ & $\begin{array}{l}\text { MDD- } \\
(n=52)\end{array}$ & $\begin{array}{c}\text { ACE } \\
(n=22)\end{array}$ & $\begin{array}{c}\text { CON } \\
(n=37)\end{array}$ & H- Wert & $\begin{array}{c}\mathrm{p}-\text { Wert } \\
(\text { Alle } \mathrm{p}<.01)\end{array}$ \\
\hline GES & $\begin{array}{c}62,9 \\
(14,9)\end{array}$ & $\begin{array}{c}42,7 \\
(12,2)\end{array}$ & $\begin{array}{c}67 \\
(17,47) \\
\end{array}$ & $\begin{array}{l}30,9 \\
(6,8)\end{array}$ & 80,91 & $\mathrm{a}$ \\
\hline EM & $\begin{array}{l}16,6 \\
(5,2)\end{array}$ & $\begin{array}{l}10,64 \\
(5)\end{array}$ & $\begin{array}{l}15,8 \\
(5,1)\end{array}$ & $\begin{array}{c}6,6 \\
(2,5)\end{array}$ & 63,72 & $\mathrm{a}$ \\
\hline $\mathbf{K M}$ & $\begin{array}{c}9,7 \\
(4,8)\end{array}$ & $\begin{array}{c}5,8 \\
(1,6)\end{array}$ & $\begin{array}{l}12,9 \\
(5,5)\end{array}$ & $\begin{array}{c}5,4 \\
(1,1)\end{array}$ & 64,54 & $\mathrm{c}$ \\
\hline SM & $\begin{array}{c}10,7 \\
(6,7)^{1}\end{array}$ & $\begin{array}{c}5,5 \\
(1,2)\end{array}$ & $\begin{array}{l}11,1 \\
(6)^{2}\end{array}$ & $\begin{array}{c}5,1 \\
(0,4)^{3}\end{array}$ & 49,07 & $\mathrm{c}$ \\
\hline EV & $\begin{array}{l}16,2 \\
(4,2)\end{array}$ & $\begin{array}{l}13,4 \\
(5,2)\end{array}$ & $\begin{array}{l}17,1 \\
(4,1)\end{array}$ & $\begin{array}{c}8,1 \\
(3,3)\end{array}$ & 56,31 & $\mathrm{~b}$ \\
\hline $\mathbf{K V}$ & $\begin{array}{l}10,1 \\
(3,5)\end{array}$ & $\begin{array}{c}7,3 \\
(2,5)\end{array}$ & $\begin{array}{l}10,6 \\
(4,2)\end{array}$ & $\begin{array}{c}5,9 \\
(1,5)\end{array}$ & 43,63 & $\mathrm{c}$ \\
\hline
\end{tabular}

Ergebnisse sind präsentiert als Mittelwerte und Standardabweichung einschließlich $H$ - und p-Werte des Kruskal-Wallis-Tests. MDD+: Patientinnen mit frübkindlicher Traumatisierung und Depression, MDD-: depressive Patientinnen obne frübkindliche Traumatisierung, ACE: gesunde Probandinnen mit frübkindlicher Traumatisierung, CON: Kontrollgruppe. ETI: Early Trauma Inventory, GES: Gesamtskala, GT: generelles Trauma, EKB: erzieherische/körperliche Bestrafung, EE: emotionale Erfahrungen, SE: sexuelle Erfahrungen, CTQ: Child Trauma Questionnaire, EM: emotionaler Missbrauch, KM: körperlicher Missbrauch, SM: sexueller Missbrauch, EV: emotionale Vernachlässigung, KV: körperliche Vernachlässigung. ${ }^{1} n=29$, ${ }^{2} n=21, \quad{ }^{3} n=35, \quad a: M D D+=A C E>M D D->C O N ; \quad b: M D D+=A C E=M D D->C O N$, $c: M D D+=A C E>M D D-=C O N$ 

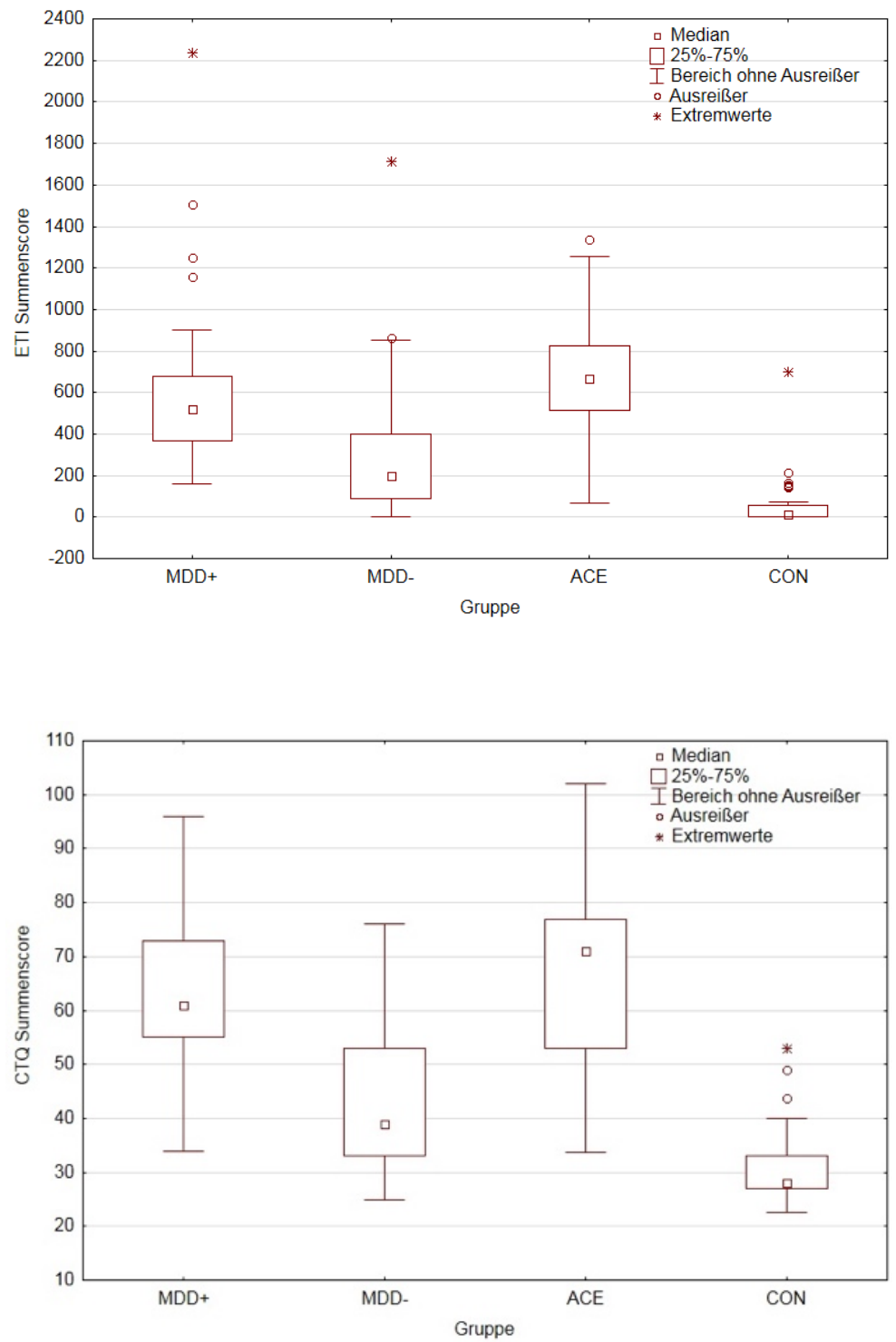

Abbildung 8: Ergebnisse des ETI- und CTQ-Summenscores der vier Versuchsgruppen als BoxWhisker-Plot

MDD+: Patientinnen mit frübkindlicher Traumatisierung und Depression, MDD-: depressive Patientinnen obne frübkindliche Traumatisierung, ACE: gesunde Probandinnen mit frübkindlicher Traumatisierung, CON: Kontrollgruppe 


\subsubsection{Depressivität}

Die Auswertung der beiden Fragebögen zur Erfassung von Depressionen, dem BDI-II und der MADRS, ergab folgendes Bild: Die Teilnehmerinnen der beiden Gruppen MDD+ und MDD- wiesen erhöhte Werte auf, welche einer mittelschweren Depression entsprachen. Sie zeigten signifikant höhere Werte als die anderen beiden Versuchsgruppen ACE und CON. Die Teilnehmerinnen der Versuchsgruppen ACE und CON wiesen in ihren mittleren Werten keine Depression auf. Die genaue Auswertung der Depressionsfragebögen ist in Tabelle 7 dargestellt. Abbildung 9 zeigt die Auswertung der Gesamtscores der einzelnen Versuchsgruppen im MARDS und im BDI-II als Box-Whisker-Plot.

Tabelle 7: Ergebnisse der MADRS und des BDI-II differenziert nach Versuchsgruppe

\begin{tabular}{|l|cccccc|}
\hline & $\begin{array}{c}\text { MDD+ } \\
(\mathbf{n}=\mathbf{3 2})\end{array}$ & $\begin{array}{c}\text { MDD - } \\
(\mathbf{n}=\mathbf{5 2})\end{array}$ & $\begin{array}{c}\text { ACE } \\
(\mathbf{n}=\mathbf{2 0})\end{array}$ & $\begin{array}{c}\text { CON } \\
(\mathbf{n}=\mathbf{3 6})\end{array}$ & H-Wert & p-Wert \\
\hline MADRS & 23,3 & 21,3 & 8,9 & 2,8 & 110,02 & $<.01^{\mathrm{a}}$ \\
& $(11,2)$ & $(10,4)$ & $(7,3)$ & $(3)$ & & \\
\hline & $\mathbf{M D D}+$ & $\mathbf{M D D}-$ & $\mathbf{A C E}$ & $\mathbf{C O N}$ & H-Wert & p-Wert \\
& $(\mathbf{n}=\mathbf{3 2})$ & $(\mathbf{n}=\mathbf{5 2})$ & $(\mathbf{n}=\mathbf{2 2})$ & $(\mathbf{n}=\mathbf{3 7})$ & & \\
\hline BDI-II & 31,8 & 27,5 & 2,8 & 0,6 & 77,17 & $<.01^{\mathrm{a}}$ \\
& $(6,8)$ & $(6,8)$ & $(2,3)$ & $(1)$ & & \\
\hline
\end{tabular}

Ergebnisse sind präsentiert als Mittelwerte und Standardabweichung einschließlich $H$ - und $p$-Werte des Kruskal-Wallis-Tests. MDD+: Patientinnen mit frühkindlicher Traumatisierung und Depression, MDD-: depressive Patientinnen obne frühkindliche Traumatisierung, ACE: gesunde Probandinnen mit frühkindlicher Traumatisierung, CON: Kontrollgruppe, MADRS: Montgomery-Asberg Depression Rating Scale, BDI-II: Beck Depressions-Inventar-II, a: MDD+=MDD->ACE $=C O N$ 

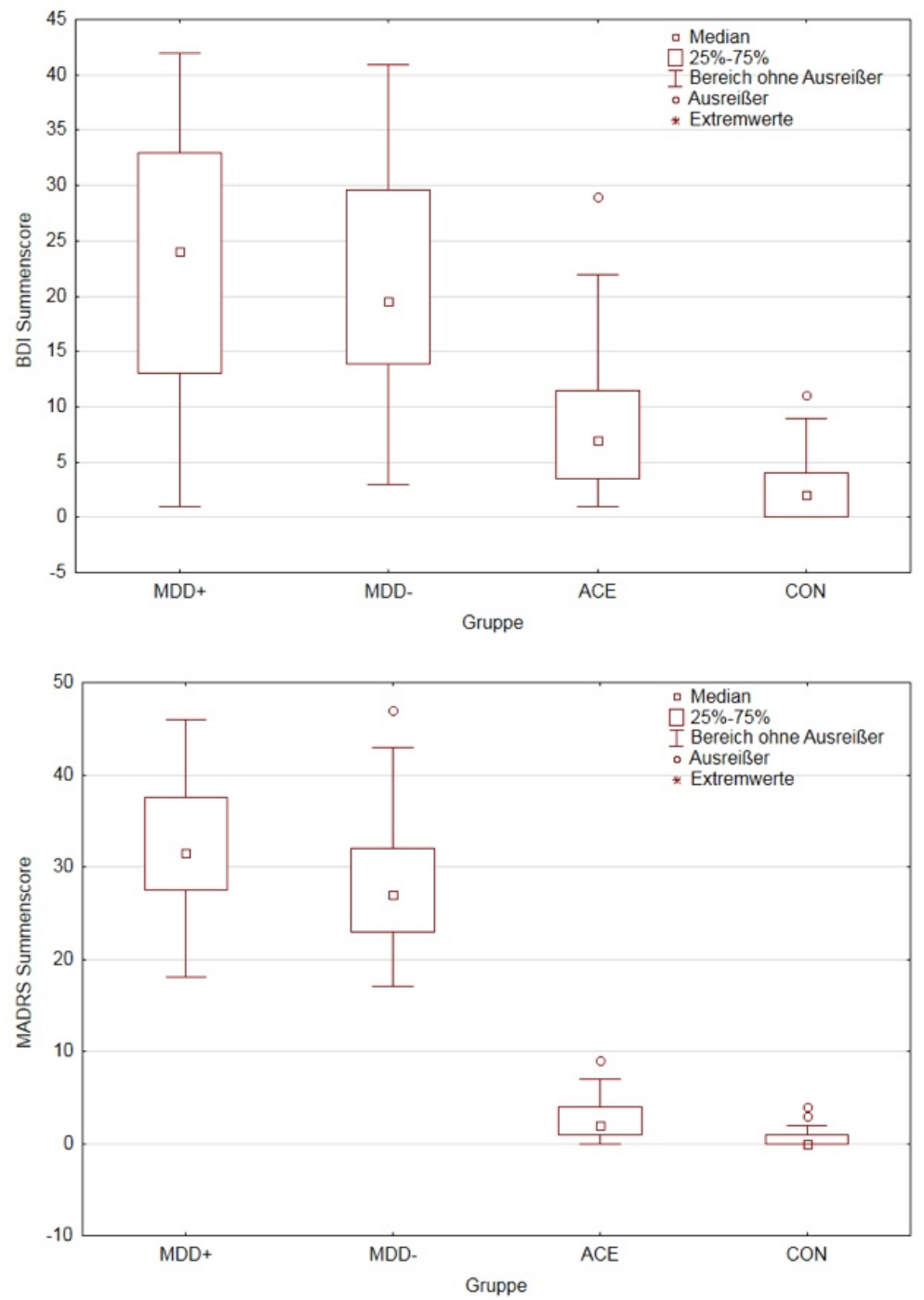

Abbildung 9: Ergebnisse des BDI- und MADRS-Summenscores der vier Versuchsgruppen als BoxWhisker-Plot

MDD+: Patientinnen mit frübkindlicher Traumatisierung und Depression, MDD-: depressive Patientinnen obne frübkindliche Traumatisierung, ACE: gesunde Probandinnen mit frühkindlicher Traumatisierung, CON: Kontrollgruppe 


\subsection{Trier Social Stress Test}

\subsubsection{Kardiovaskuläre Stressreaktion}

In den folgenden Unterkapiteln werden die Versuchsparameter sBD, dBD und HF deskriptiv ausgewertet. Zudem wurde ermittelt, ob es im Gesamtkollektiv und in den einzelnen Versuchsgruppen zu einer signifikanten Veränderung der Messparameter zwischen den einzelnen Versuchszeitpunkten im Sinne einer Stressreaktivität, Recovery 1 und 2 gekommen war. In Tabelle 8 werden detaillierte Informationen zu den Mittelwerten und Standardabweichungen der kardiovaskulären Parameter gegeben sowie auch die Ergebnisse der Einstichproben-t-Tests dargestellt. Eine exakte Aufschlüsselung der ermittelten p-Werte ist in der Tabelle A1 im Anhang einsehbar. Insgesamt fiel über alle Hypothesen und Versuchsgruppen hinweg eine große Standardabweichung auf, weshalb der prozentuale Anteil positiver und negativer Veränderungen des sBD, des dBD und der HF für die kardiovaskuläre Stressreaktivität, Recovery 1 und 2 ermittelt wurde. Dies ist in Tabelle 9 detailliert dargestellt. 
Tabelle 8: Auswertung des TSST: systolische und diastolische Blutdruck- sowie Pulswerte der Baseline, Stressreaktivität, Recovery 1 und 2

\begin{tabular}{|c|c|c|c|c|c|}
\hline & $\begin{array}{c}\text { Gesamt } \\
(n=143)\end{array}$ & $\begin{array}{l}\text { MDD+ } \\
(\mathrm{n}=32)\end{array}$ & $\begin{array}{l}\text { MDD- } \\
(n=52)\end{array}$ & $\begin{array}{c}\text { ACE } \\
(n=22)\end{array}$ & $\begin{array}{c}\text { CON } \\
(n=37)\end{array}$ \\
\hline & $M(S D)$ & $M(S D)$ & $M(S D)$ & $M(S D)$ & $M(S D)$ \\
\hline \multicolumn{6}{|l|}{ Systolischer RR } \\
\hline Baseline (mmHg) & $\begin{array}{l}121,3 \\
(13,1)\end{array}$ & $\begin{array}{l}119,8 \\
(14,4)\end{array}$ & $\begin{array}{l}124,3 \\
(14,3)\end{array}$ & $\begin{array}{c}116,7 \\
(8,6)\end{array}$ & $\begin{array}{l}121,1 \\
(11,7)\end{array}$ \\
\hline Reaktivität (\%) & $\begin{array}{l}+6,8^{1} \\
(11,3)\end{array}$ & $\begin{array}{l}+7,3 \\
(9,9)\end{array}$ & $\begin{array}{l}+6,8^{2} \\
(13,9)\end{array}$ & $\begin{array}{l}+5,1 \\
(9,8)\end{array}$ & $\begin{array}{l}+7,4 \\
(9,2)\end{array}$ \\
\hline Recovery $1(\%)$ & $\begin{array}{l}-3,9^{1} \\
(9,1)\end{array}$ & $\begin{array}{l}-4,8 \\
(6,9)\end{array}$ & $\begin{array}{l}-4,5^{2} \\
(11,9)\end{array}$ & $\begin{array}{l}-1,8 \\
(9,0)\end{array}$ & $\begin{array}{l}-3,4 \\
(6,0)\end{array}$ \\
\hline Recovery $2(\%)$ & $\begin{array}{l}+2,0 \\
(8,2)\end{array}$ & $\begin{array}{r}+2,0 \\
(10,1)\end{array}$ & $\begin{array}{l}+0,9 \\
(8,6)\end{array}$ & $\begin{array}{l}+2,6 \\
(7,0)\end{array}$ & $\begin{array}{l}+3,3 \\
(6,1)\end{array}$ \\
\hline \multicolumn{6}{|l|}{ Diastolischer RR } \\
\hline Baseline (mmHg) & $\begin{array}{c}82,3 \\
(10,1)\end{array}$ & $\begin{array}{l}79,8 \\
(10)\end{array}$ & $\begin{array}{c}85,1 \\
(10,1)\end{array}$ & $\begin{array}{l}79,5 \\
(9,6)\end{array}$ & $\begin{array}{l}82,1 \\
(9,8)\end{array}$ \\
\hline Reaktivität (\%) & $\begin{array}{c}+7,1^{1} \\
(12,9)\end{array}$ & $\begin{array}{c}+8,0 \\
(12,8)\end{array}$ & $\begin{array}{l}+8,8^{2} \\
(16,8)\end{array}$ & $\begin{array}{l}+5,2 \\
(9,8)\end{array}$ & $\begin{array}{l}+5,0 \\
(7,9)\end{array}$ \\
\hline Recovery $1(\%)$ & $\begin{array}{l}-2,9^{1} \\
(9,9)\end{array}$ & $\begin{array}{l}-4,2 \\
(8,9)\end{array}$ & $\begin{array}{c}-2,7 \\
(11,6)\end{array}$ & $\begin{array}{l}-3,0 \\
(9,2)\end{array}$ & $\begin{array}{l}-1,9 \\
(8,7)\end{array}$ \\
\hline Recovery $2(\%)$ & $\begin{array}{l}+3,2 \\
(9,6)\end{array}$ & $\begin{array}{l}+2,8 \\
(9,9)\end{array}$ & $\begin{array}{c}+4,6 \\
(10,8)\end{array}$ & $\begin{array}{l}+1,7 \\
(9,7)\end{array}$ & $\begin{array}{l}+2,6 \\
(7,5)\end{array}$ \\
\hline \multicolumn{6}{|l|}{ Herzfrequenz } \\
\hline Baseline (mmHg) & $\begin{array}{l}75,4^{1} \\
(12,1)\end{array}$ & $\begin{array}{c}77,4 \\
(14,4)\end{array}$ & $\begin{array}{c}74,5 \\
(11,3)\end{array}$ & $\begin{array}{l}76,3^{3} \\
(11,8)\end{array}$ & $\begin{array}{c}74,5 \\
(11,4)\end{array}$ \\
\hline Reaktivität (\%) & $\begin{array}{l}+3,0^{4} \\
(10,7)\end{array}$ & $\begin{array}{l}+2,2 \\
(9,3)\end{array}$ & $\begin{array}{l}+5,7^{2} \\
(10,0)\end{array}$ & $\begin{array}{l}+2,4^{3} \\
(9,3)\end{array}$ & $\begin{array}{l}+0,4 \\
(12,9)\end{array}$ \\
\hline Recovery $1(\%)$ & $\begin{array}{l}-3,8^{4} \\
(8,1)\end{array}$ & $\begin{array}{l}-2,5 \\
(6,5)\end{array}$ & $\begin{array}{l}-4,3^{2} \\
(8,8)\end{array}$ & $\begin{array}{l}-4,2^{3} \\
(6,8)\end{array}$ & $\begin{array}{l}-3,8 \\
(9,1)\end{array}$ \\
\hline Recovery $2(\%)$ & $\begin{array}{l}-1,4^{1} \\
(8,8)\end{array}$ & $\begin{array}{l}-0,6 \\
(8,5)\end{array}$ & $\begin{array}{l}+0,4 \\
(8,5)\end{array}$ & $\begin{array}{l}-2,3^{3} \\
(7,2)\end{array}$ & $\begin{array}{l}-4,1 \\
(9,8)\end{array}$ \\
\hline
\end{tabular}

Ergebnisse sind präsentiert als Mittelwerte und Standardabweichung. Signifikante prozentuale Veränderungen der Parameter in den Einstichproben-t-Tests gegen 0 sind fett gedruckt. MDD+: Patientinnen mit frübkindlicher Traumatisierung und Depression, MDD-: depressive Patientinnen ohne frühkindliche Traumatisierung, ACE: gesunde Probandinnen mit frühkindlicher Traumatisierung, CON: Kontrollgruppe, Reaktivität: prozentuale Differenz der kardiovaskulären Parameter von t2 (vor dem Stress) $\rightarrow$ t3 (direkt nach dem TSST), Recovery 1: prozentuale Differenz der kardiovaskulären Parameter von t3 (direkt nach dem TSST) $\rightarrow$ t4 (nach der Erholungsphase), Recovery 2: prozentuale Differenz der kardiovaskulären Parameter im Vergleich von $t 2$ (vor dem Stress) $\rightarrow t 4$ (nach der Erholungsphase). ${ }^{1} n=142 ;{ }^{2} n=51,{ }^{3} n=21,{ }^{4} n=141$ 
Tabelle 9: Anteil positiver und negativer prozentualer Veränderungen der kardiovaskulären Parameter des Gesamtkollektivs und der einzelnen Versuchsgruppen für die kardiovaskuläre Stressreaktivität, Recovery 1 und 2 des sBD, $d B D$ und der $H F$

\begin{tabular}{|c|c|c|c|c|c|c|}
\hline & & $\begin{array}{c}\text { Gesamt } \\
(n=143)\end{array}$ & $\begin{array}{l}\text { MDD+ } \\
(n=32)\end{array}$ & $\begin{array}{l}\text { MDD- } \\
(\mathrm{n}=52)\end{array}$ & $\begin{array}{c}\text { ACE } \\
(n=22)\end{array}$ & $\begin{array}{c}\text { CON } \\
(\mathrm{n}=37)\end{array}$ \\
\hline \multicolumn{7}{|c|}{ Systolischer BD } \\
\hline \multirow[t]{2}{*}{ Reaktivität } & - & 27,5 & 28,1 & 29,4 & 31,8 & 21,6 \\
\hline & + & 72,5 & 71,9 & 70,6 & 68,2 & 78,4 \\
\hline \multirow[t]{2}{*}{ Recovery 1} & - & 71,1 & 78,1 & 72,5 & 54,5 & 73,0 \\
\hline & + & 28,9 & 21,9 & 27,5 & 45,5 & 27,0 \\
\hline \multirow[t]{2}{*}{ Recovery 2} & - & 38,2 & 40,6 & 44,2 & 31,8 & 32,4 \\
\hline & + & 61,8 & 59,4 & 55,8 & 68,2 & 67,6 \\
\hline \multicolumn{7}{|c|}{ Diastolischer BD } \\
\hline \multirow[t]{2}{*}{ Reaktivität } & - & 26,8 & 21,9 & 25,5 & 27,3 & 32,4 \\
\hline & + & 73,2 & 78,1 & 74,5 & 72,7 & 67,6 \\
\hline \multirow[t]{2}{*}{ Recovery 1} & - & 62,0 & 74,5 & 54,9 & 68,2 & 56,8 \\
\hline & + & 38,0 & 24,5 & 45,1 & 31,8 & 43,2 \\
\hline \multirow[t]{2}{*}{ Recovery 2} & - & 38,5 & 37,5 & 36,5 & 45,5 & 32,5 \\
\hline & + & 61,5 & 62,5 & 63,5 & 54,5 & 67,6 \\
\hline \multicolumn{7}{|c|}{ Herzfrequenz } \\
\hline \multirow[t]{2}{*}{ Reaktivität } & - & 39,7 & 46,9 & 29,4 & 38,1 & 48,7 \\
\hline & + & 60,3 & 53,1 & 70,6 & 61,9 & 51,3 \\
\hline \multirow[t]{2}{*}{ Recovery 1} & - & 68,1 & 71,9 & 64,7 & 76,2 & 64,9 \\
\hline & + & 31,9 & 28,1 & 35,3 & 23,8 & 35,1 \\
\hline \multirow[t]{2}{*}{ Recovery 2} & - & 59,2 & 59,4 & 50,0 & 71,4 & 64,9 \\
\hline & + & 40,8 & 49,6 & 50,0 & 28,6 & 35,1 \\
\hline
\end{tabular}

Ergebnisse sind präsentiert in Prozentangaben (\%). MDD+: Patientinnen mit frübkindlicher Traumatisierung und Depression, MDD-: depressive Patientinnen obne frübkindliche Traumatisierung, ACE: gesunde Probandinnen mit frübkindlicher Traumatisierung, CON: Kontrollgruppe, Reaktivität:prozentuale Differenz der kardiovaskulären Parameter von t2 (vor dem Stress) $\rightarrow$ t3 (direkt nach dem TSST), Recovery 1: prozentuale Differenz der kardiovaskulären Parameter von t3 (direkt nach dem TSST) $\rightarrow$ t4 (nach der Erholungsphase), Recovery 2: prozentuale Differenz der kardiovaskulären Parameter im Vergleich von $t 2$ (vor dem Stress) $\rightarrow t 4$ (nach der Erholungsphase). -: prozentualer Anteil der Probandinnen, die einen Abfall der kardiovaskulären Parameter in den negativen Bereich zeigten, +: prozentualer Anteil der Probandinnen, die einen Anstieg der kardiovaskulären Parameter in den positiven Bereich zeigten

\subsubsection{Systolischer Blutdruck}

Um einen Eindruck über die Entwicklung des sBD während des Versuchs zu erhalten, wurde eine Verlaufsgraphik erstellt (siehe Abbildung 10). Im Gesamtkollektiv stieg der sBD in Reaktion auf den TSST gemessen von $\mathrm{t} 2 \rightarrow \mathrm{t} 3$ im Mittel um $+6,8 \pm 11,3 \%$ an; dieser Anstieg zeigte sich für das Gesamtkollektiv signifikant. In der Aufschlüsselung für die einzelnen Versuchsgruppen konnte diese Signifikanz jedoch lediglich für die Versuchsgruppen 
MDD+, MDD- und CON bestätigt werden (siehe Tabelle 8). Insgesamt zeigten 72,5\% der Probandinnen einen Anstieg, 27,5 \% einen Abfall des sBD in Reaktion auf den TSST (siehe Tabelle 9).

Nach der Erholungsphase (t4) wies die Gesamtheit der Probandinnen im Vergleich zur Stresseinwirkung (t3) eine statistisch relevante Abnahme des sBD um $-3,9 \pm 9,1 \%$ auf. Bei der Betrachtung der Untergruppen erreichte dies jedoch lediglich für die Versuchsgruppen MDD+ und CON statistische Signifikanz. Im zeigten Gesamtkollektiv 28,9 \% der Probandinnen einen weiteren Anstieg des sBD nach der Erholungsphase, bei 71,1\% der Probandinnen war der sBD abgesunken.

Verglichen mit der Baseline ( $\mathrm{t} 2$ ) war der mittlere sBD des Gesamtkollektivs nach der Erholungsphase ( $\mathrm{t} 4$ ) noch signifikant um $+2 \pm 8,2 \%$ erhöht. Diese statistische Relevanz konnte in der Aufschlüsselung der Versuchsgruppen jedoch nur für die CON nachgewiesen werden. Bei insgesamt 61,8\% der Probandinnen war der sBD bei t4 im Vergleich zu t2 noch erhöht, 38,2 \% zeigten niedrigere Werte als in der Baseline.

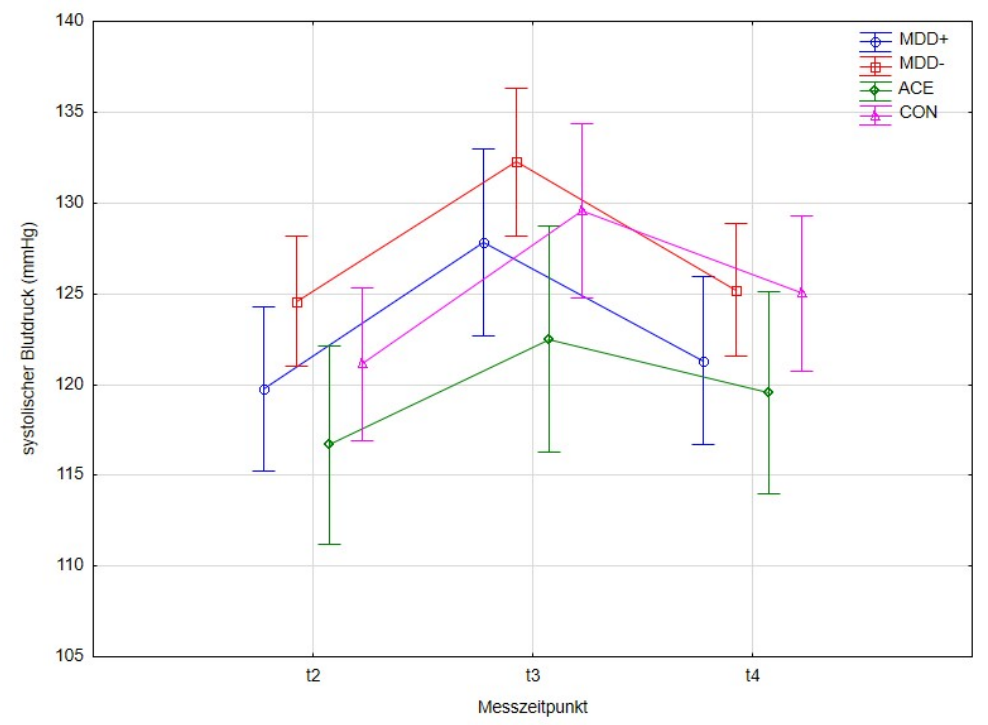

Abbildung 10: Verlauf der systolischen Blutdruckwerte der einzelnen Versuchsgruppen über t2 (vor dem Stress, 0 min), t3 (direkt nach dem Stress, +15 min) und t4 (nach der Erholungsphase, +25 min) MDD+: Patientinnen mit frübkindlicber Traumatisierung und Depression, MDD-: depressive Patientinnen obne frübkindliche Traumatisierung, ACE: gesunde Probandinnen mit frübkindlicher Traumatisierung, CON: Kontrollgruppe

\subsubsection{Diastolischer Blutdruck}

Der Verlauf des dBD wird in Abbildung 11 dargestellt. Der mittlere Anstieg des dBD des Gesamtkollektivs betrug $+7,1 \pm 12,9 \%$ von $\mathrm{t} 2 \rightarrow \mathrm{t} 3$, dieser Anstieg war für das Gesamtkollektiv signifikant. Bei Betrachtung der einzelnen Versuchsgruppen verfehlte lediglich die Gruppe ACE die statistische Relevanz dieser Beobachtung. Insgesamt zeigten 73,3 \% der 
Probandinnen einen Anstieg des dBD in Reaktion auf den TSST, 26,8\% reagierten mit einem Abfall desselben.

Nach der Erholungsphase sank der dBD von t3 $\rightarrow$ t4 im Mittel um -2,9 \pm 9,9\% ab. In der Gesamtheit war dieser Abfall signifikant, konnte jedoch in der Analyse der einzelnen Versuchsgruppen nicht gefunden werden. Bei insgesamt $62 \%$ der Probandinnen war der dBD nach der Erholungsphase abgesunken, $38 \%$ zeigten jedoch einen weiteren Anstieg des dBD.

Verglichen mit der Baseline (t2) war der dBD nach der Erholungsphase (t4) im Durchschnitt in der Gesamtheit um +3,2 \pm 9,6 \% erhöht. Dieser prozentuale Unterschied zeigte sich für das Gesamtkollektiv, bei Betrachtung der Untergruppen nur für die Gruppe MDD-, signifikant. Bei 61,5 \% der Probandinnen war der dBD nach der Erholungsphase niedriger als zu Beginn des Versuchs, in 38,5 \% der Fälle noch erhöht.

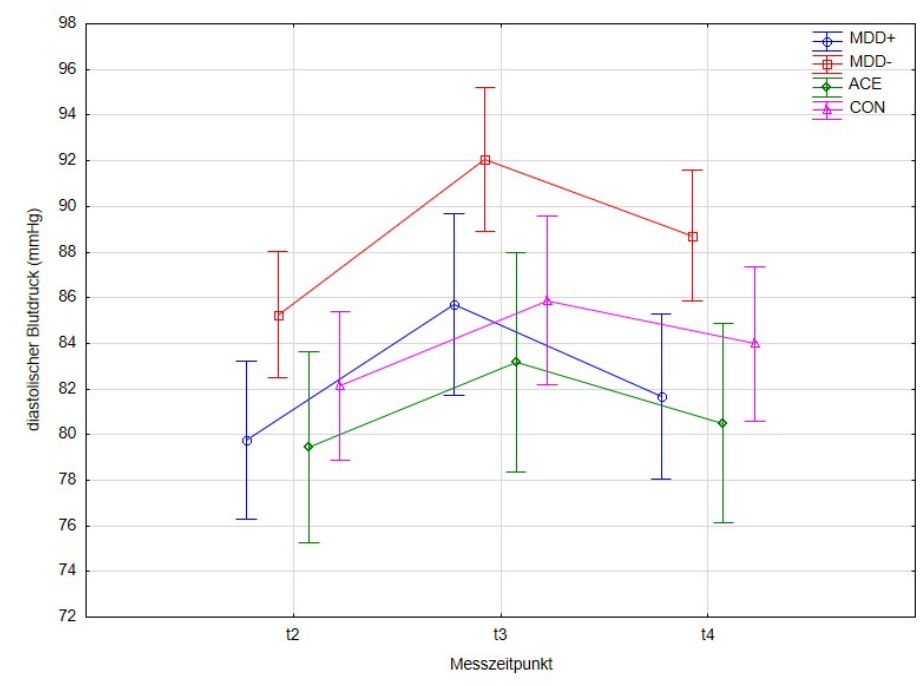

Abbildung 11: Verlauf der diastolischen Blutdruckwerte der einzelnen Versuchsgruppen über t2 (vor dem Stress, 0 min), t3 (direkt nach dem Stress, +15 min) und t4 (nach der Erholungsphase, +25 min) MDD+: Patientinnen mit frübkindlicher Traumatisierung und Depression, MDD-: depressive Patientinnen obne frübkindliche Traumatisierung, ACE: gesunde Probandinnen mit frübkindlicher Traumatisierung, CON: Kontrollgruppe

\subsubsection{Herzfrequenz}

Der Verlauf der HF innerhalb des Versuchs ist in Abbildung $12 \mathrm{zu}$ sehen. Im Gesamtkollektiv stieg die Herzfrequenz signifikant um $+3,0 \pm 10,7 \%$ an. Bei der Betrachtung der einzelnen Versuchsgruppen war dieser Anstieg lediglich für die Versuchsgruppe MDDsignifikant; die Gruppen MDD+, ACE und CON zeigten keinen statistisch relevanten Anstieg der HF in Reaktion auf den TSST. Dieses Phänomen spiegelt sich auch in dem Anteil positiver und negativer prozentualer Veränderungen der HF der einzelnen Versuchsgruppen wider: In der Gruppe MDD- zeigten 70,6 \% der Probandinnen einen An- 
stieg der HF in Reaktion auf den TSST, in den restlichen Versuchsgruppen schwankt der Anteil positiver Veränderungen auf den TSST zwischen 51,4 bis maximal $62 \%$.

Nach der Erholungsphase (t4) ging die durchschnittliche HF verglichen zur Stresseinwirkung (t3) im Gesamtkollektiv von $\mathrm{t} 3 \rightarrow \mathrm{t} 4$ signifikant um $-3,8 \pm 8,1 \%$ zurück, wobei die statistische Relevanz auch hierbei lediglich in der Untergruppe MDD- gegeben war. Im Gesamtkollektiv zeigten 68,1\% der Probandinnen einen Abfall, 31,2 \% einen weiteren Anstieg der HF von $\mathrm{t} 3 \rightarrow \mathrm{t} 4$.

Die Recovery 2 der HF zeigte folgendes Bild: Verglichen mit der Baseline (t2) wies das Gesamtkollektiv nach der Erholungsphase (t4) zwar im Mittel um -1,4 \pm 8,8 \% niedrigere Pulswerte auf. Dieser Unterschied zeigte sich jedoch allseits statistisch nicht relevant. In 40,9 \% der Fälle war die HF nach der Erholungsphase noch erhöht, in 59,1 \% niedriger als zu Beginn des Versuchs.

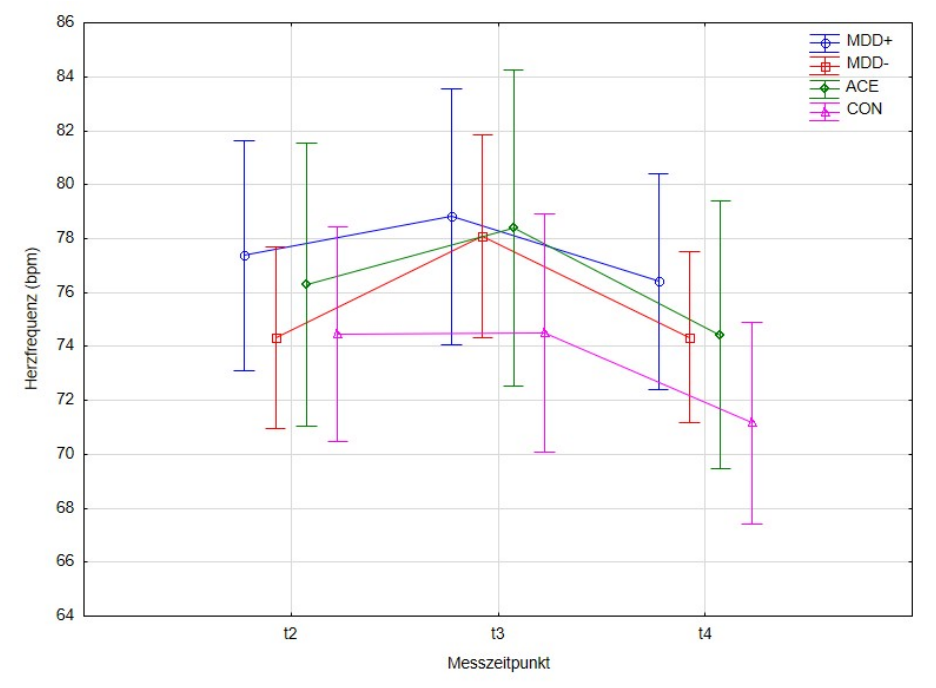

Abbildung 12: Verlauf der Herafrequenz der einzelnen Versuchsgruppen über t2 (vor dem Stress, 0 min), t3 (direkt nach dem Stress, +15 min) und t4 (nach der Erholungsphase, +25 min) MDD+: Patientinnen mit frübkindlicher Traumatisierung und Depression, MDD-: depressive Patientinnen obne frübkindliche Traumatisierung, ACE: gesunde Probandinnen mit frübkindlicher Traumatisierung, CON: Kontrollgruppe

\subsection{2 Überprüfen der Hypothesen: Einfluss von Depression und Kindheitstrauma auf die kardiovaskuläre Stressreaktivität und Recovery}

Um zu überprüfen, ob sich die vier Versuchsgruppen bezüglich der Stressreaktivität, Recovery 1 und 2 der kardiovaskulären Parameter sBD, dBD und HF unterscheiden, wurden zum Gruppenvergleich einfaktorielle Varianzanalysen durchgeführt. Die Ergebnisse sind in Tabelle 10 dargestellt. 
Tabelle 10: Gruppenvergleich der Stressreaktivität, Recovery 1 und 2 für die kardiovaskulären Parameter $s B D, d B D$ und $H F$

\begin{tabular}{|l|cccccc|}
\hline & \multicolumn{2}{|c}{$\begin{array}{c}\text { Systolischer } \\
\text { Blutdruck }\end{array}$} & \multicolumn{2}{c|}{$\begin{array}{c}\text { Diastolischer } \\
\text { Blutdruck }\end{array}$} & \multicolumn{2}{c|}{ Herzfrequenz } \\
& $\mathrm{p}-$ Wert & F-Wert & $\mathrm{p}$-Wert & F-Wert & $\mathrm{p}$-Wert & F-Wert \\
\hline Reaktivität & .89 & 0,21 & .47 & 0,85 & .13 & 1,90 \\
\hline Recovery 1 & .64 & 0,56 & .80 & 0,33 & .79 & 0,35 \\
\hline Recovery 2 & .57 & 0,67 & .60 & 0,63 & .10 & 2,11 \\
\hline
\end{tabular}

Ergebnisse präsentieren den Gruppenvergleich der kardiovaskulären Werte mittels einfaktorieller ANOVA, dargestellt als Fund p-Werte. Reaktivität: prozentuale Differenz der kardiovaskulären Parameter von t2 (vor dem Stress) $\rightarrow$ t3 (direkt nach dem TSST), Recovery 1: prozentuale Differenz der kardiovaskulären Parameter von t3 (direkt nach dem TSST) $\rightarrow$ t4 (nach der Erholungsphase), Recovery 2: prozentuale Differenz der kardiovaskulären Parameter im Vergleich von t2 (vor dem Stress) $\rightarrow$ t4 (nach der Erholungsphase)

\subsubsection{Erste Hypothese: Depression, Kindheitstrauma und die kardiovaskuläre Stressreaktivität}

Im Gruppenvergleich konnten keine statistischen Unterschiede für die Reaktivität des sBD $(\mathrm{F}=0,21 ; \mathrm{p}=.89)$, des $\mathrm{dBD}(\mathrm{F}=0,85 ; \mathrm{p}=.47)$ und der $\operatorname{HF}(\mathrm{F}=1,90 ; \mathrm{p}=.13)$ gefunden werden (siehe Tabelle 10). Eine Darstellung der KVR als prozentuale Veränderung des $\mathrm{sBD}, \mathrm{dBD}$ und der HF von $\mathrm{t} 2 \rightarrow 3$ differenziert nach Gruppe als Box-Whisker-Plot zeigt Abbildung 13. 

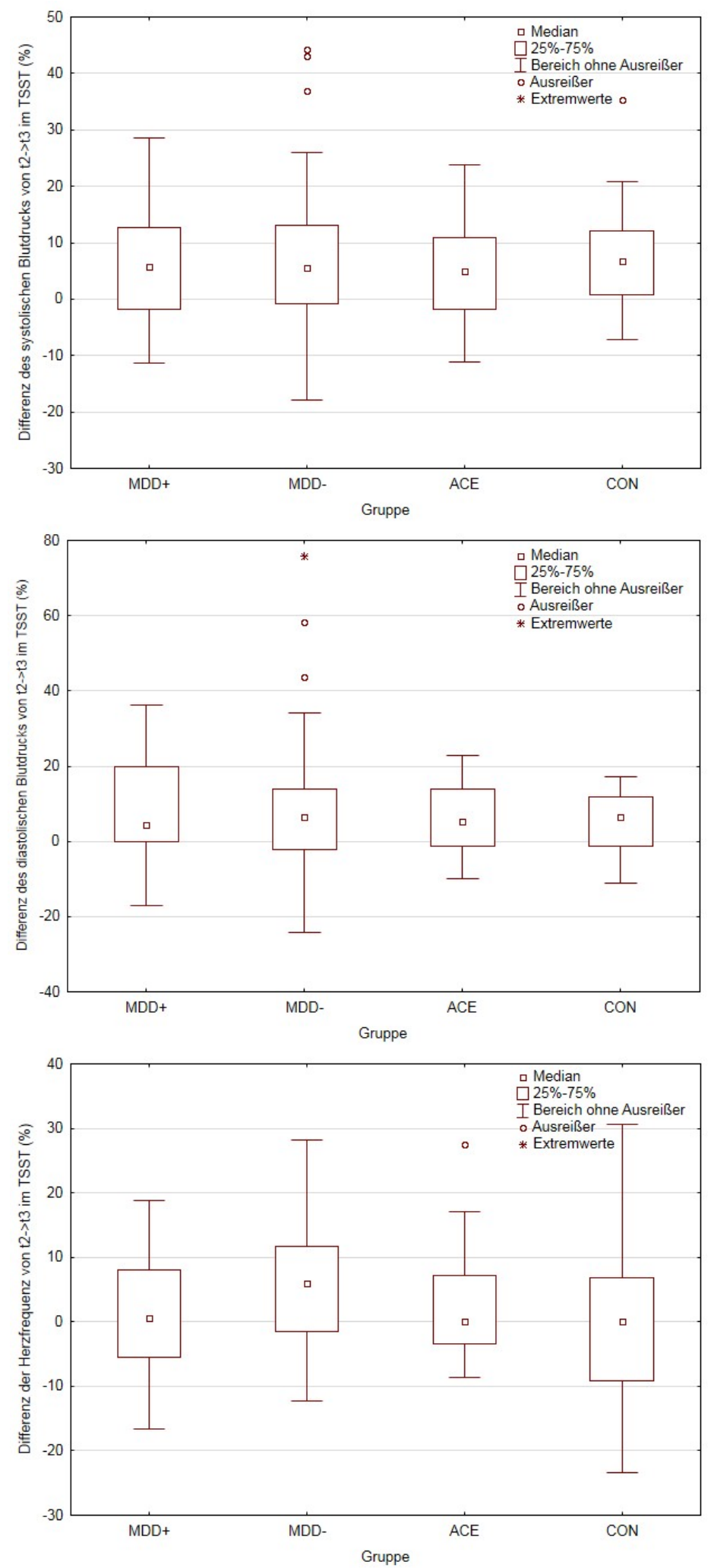

Abbildung 13: Darstellung der Stressreaktivität als prozentuale Veränderung des diastolischen und systolischen Blutdrucks sowie der Herafrequenz von t2 (vor dem Stress, 0 min) $\rightarrow$ t3 (direkt nach dem Stress, +15 min) im TSST nach Gruppenzugehörigkeit als Box-Whisker-Plot MDD+: Patientinnen mit frübkindlicher Traumatisierung und Depression, MDD-: depressive Patientinnen obne frühkindliche Traumatisierung, ACE: gesunde Probandinnen mit frübkindlicher Traumatisierung, CON: Kontrollgruppe 
3.2.2.2 Zweite Hypothese: Depression, Kindheitstrauma und die kardiovaskuläre Recovery

\subsection{Recovery 1}

Beim Vergleich der Versuchsgruppen wurden keine signifikanten Unterschiede in der Recovery 1 des $\operatorname{sBD}(\mathrm{F}=0,56 ; \mathrm{p}=.64)$, des $\mathrm{dBD}(\mathrm{F}=0,33 ; \mathrm{p}=.80)$ und der HF $(\mathrm{F}=0,35 ; \mathrm{p}=.79)$ gefunden (siehe Tabelle 10$)$. Eine Darstellung der KVRec 1 als prozentuale Veränderung des sBD, dBD und der HF von $\mathrm{t} 3 \rightarrow \mathrm{t} 4$ nach Versuchsgruppe als BoxWhisker-Plot ist in Abbildung 14 dargestellt. 

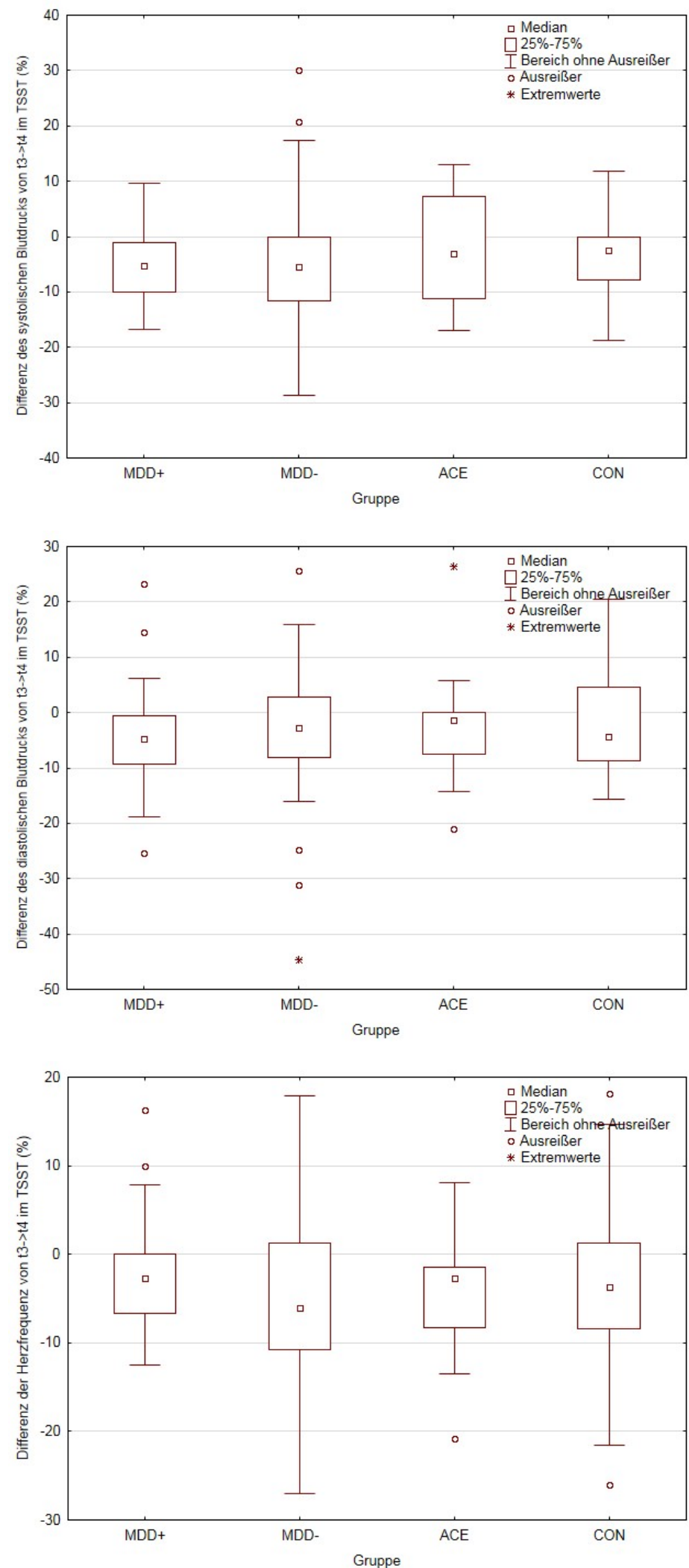

Abbildung 14: Darstellung der Recovery 1 als prozentuale Veränderung des systolischen Blutdrucks, des diastolischen Blutdrucks und der Herafrequenz von t3 (direkt nach dem Stress, +15 min) $\rightarrow$ t4 (nach der Erholungsphase, +25 min) im TSST nach Gruppenzugebörigkeit als Box-Whisker-Plot MDD+: Patientinnen mit frühkindlicher Traumatisierung und Depression, MDD-: depressive Patientinnen obne frühkindliche Traumatisierung, ACE: gesunde Probandinnen mit frübkindlicher Traumatisierung, CON: Kontrollgruppe 


\subsection{Recovery 2}

Beim Gruppenvergleich wurden keine signifikanten Unterschiede in der Recovery 2 des $\operatorname{sBD}(\mathrm{F}=0,67 ; \mathrm{p}=.57)$, des $\mathrm{dBD}(\mathrm{F}=0,63 ; \mathrm{p}=.60)$ und $\operatorname{der} \operatorname{HF}(\mathrm{F}=2,11 ; \mathrm{p}=.10)$ nachgewiesen (siehe Tabelle 10). Eine Darstellung der Recovery 2 als prozentuale Veränderung des $\mathrm{sBD}, \mathrm{dBD}$ und der $\mathrm{HF}$ von $\mathrm{t} 2 \rightarrow \mathrm{t} 4$ differenziert nach Versuchsgruppe als BoxWhisker-Plot ist in Abbildung 15 dargestellt. 

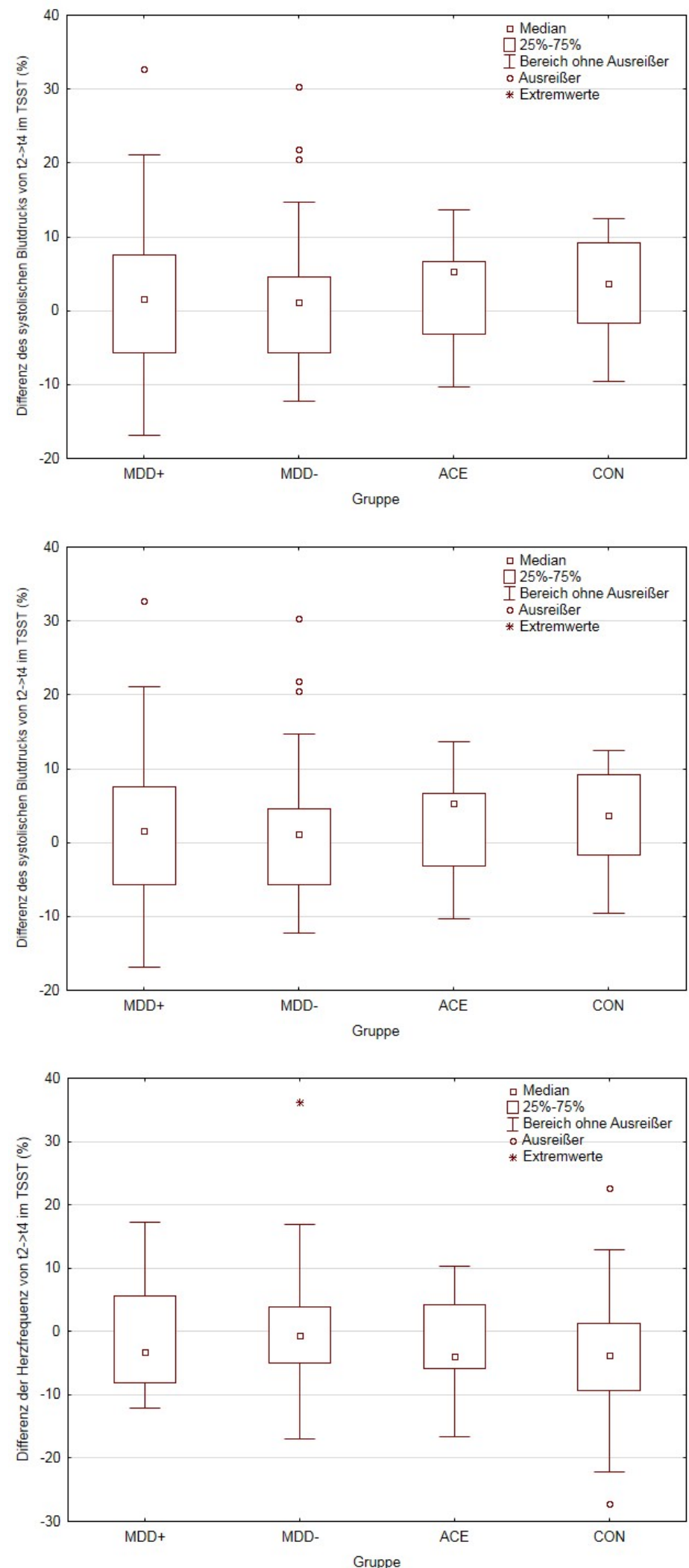

Abbildung 15: Darstellung der Recovery 2 als prozentuale Veränderung des systolischen Blutdrucks, des diastolischen Blutdrucks und der Herafrequenz, von t2 (vor dem Stress, 0 min) $\rightarrow t 4$ (nach der Erholungsphase, +25 min) im TSST nach Gruppenzugehörigkeit als Box-Whisker-Plot MDD+: Patientinnen mit frühkindlicher Traumatisierung und Depression, MDD-: depressive Patientinnen obne frühkindliche Traumatisierung, ACE: gesunde Probandinnen mit frübkindlicher Traumatisierung, CON: Kontrollgruppe 


\section{Diskussion}

\subsection{Studienziel und Resultat}

Die vorliegende Querschnittstudie hatte das Ziel, den Einfluss von Depression und Kindheitstraumata auf die kardiovaskuläre Stressreaktivität und Recovery bei Frauen systematisch $\mathrm{zu}$ untersuchen und somit mögliche Imbalancen im autonomen Nervensystem zu detektieren. Es wurden die Hypothesen aufgestellt, dass Patientinnen mit Depression und/oder Kindheitstraumata im Vergleich zur Kontrollgruppe mit einer erhöhten KVR (1. Hypothese) und einer verzögerten KVRec (2. Hypothese) auf den TSST reagieren würden. Zur Überprüfung der Annahmen wurden 143 Probandinnen in die vier Versuchsgruppen ACE, MDD+, MDD- und CON eingeteilt. Bezüglich der soziodemographischen Betrachtungen zeigten sich die vier Versuchsgruppen weitgehend homogen. In der Versuchsauswertung wurden zunächst die kardiovaskulären Stressantworten mittels Einstichproben-tTests auf Signifikanz überprüft. Für das Gesamtkollektiv zeigte sich ein signifikanter Anstieg aller gemessenen kardiovaskulären Parameter in Reaktion auf den TSST (Stressreaktivität). Bei Betrachtung der einzelnen Versuchsgruppen zeigte sich dieses Bild jedoch nicht stabil: So konnte ein signifikanter Pulsanstieg lediglich in der Gruppe MDD+ verzeichnet werden; die Gruppe ACE zeigte in keinem der Messparameter eine signifikante Stressreaktivität (siehe Kapitel 3.2.1). Nach der Erholungsphase fielen alle Verlaufsparameter im Gesamtkollektiv signifikant ab (Recovery 1). Der sBD und dBD waren nach der Erholungsphase im Vergleich zur Baseline noch signifikant erhöht; die mittleren Werte der HF hatten sich wieder dem Ausgangswert angenähert (Recovery 2). Auch hier zeigten sich in der Aufschlüsselung der Recovery der einzelnen Versuchsgruppen heterogene Ergebnisse (siehe Kapitel 3.2.1). Des Weiteren fiel über alle Versuchsgruppen und Messungen hinweg eine sehr große Standardabweichung auf. In der Aufschlüsselung der prozentualen Anteile positiver und negativer prozentualer Veränderungen der kardiovaskulären Parameter für die Stressreaktivität als auch für die Recovery 1 und 2 zeigte sich ein inhomogenes Bild. Beim Vergleich der Versuchsgruppen mittels ANOVA zeigte sich Folgendes: Für den prozentualen Anstieg der HF, sBD und dBD in Reaktion auf den TSST konnte kein signifikanter Unterscheid zwischen den Versuchsgruppen gefunden werden. Im Kontrast zu unserer ersten Hypothese hatte das Vorliegen einer Depression und/oder frühen Traumatisierung in dieser Studienkohorte keinen Einfluss auf die kardiovaskuläre Stressreaktivität. 
Abweichend von unserer 2. Hypothese konnte in unserem Versuch bei Probandinnen mit Depression und/oder frühkindlicher Traumatisierung im Vergleich zur gesunden Kontrollgruppe keine prolongierte Stressreaktion im Sinne einer verzögerten Recovery 1 und 2 der kardiovaskulären Marker HF, sBD und dBD nach der psychosozialen Stressinduktion nachgewiesen werden.

In den folgenden Kapiteln werden unsere Versuchsergebnisse in den aktuellen Forschungsstand eingeordnet und unter verschiedenen Gesichtspunkten mögliche Ursachen für die gefundenen Effekte diskutiert. Zudem werden Stärken und Schwächen der Studie herausgearbeitet und ein Ausblick für zukünftige Untersuchungen gegeben.

Es ist zu berücksichtigen, dass in den unten zitierten Studien verschiedene psychosoziale Stresstests durchgeführt wurden. Dabei muss erwähnt werden, dass auch verschiedene experimentelle psychische Stressoren teilweise unterschiedlich stark ausgeprägte kardiovaskuläre Reaktionen in der gleichen Kohorte auslösen können (Schwerdtfeger und Rosenkaimer 2011; Giles et al. 2014; Skoluda et al. 2015; Meyer et al. 2016; Yuenyongchaiwat et al. 2017). Da die Anzahl vergleichbarer Studien, welche den TSST zur experimentellen Stressinduktion nutzten, überschaubar ist, wurden auch Arbeiten mit verschiedenen psychosozialen Stresstests in die Diskussion eingeschlossen. Eine Übersicht über die angewandten Stresstests in den verschiedenen Studien wird in Tabelle A2 im Anhang gegeben.

\subsection{Einordnung der Ergebnisse in den aktuellen Forschungsstand}

\subsubsection{Hypothese: Depression, Kindheitstrauma und kardiovaskuläre Stressreaktivität}

Im Kontrast zu unserer ersten Hypothese hatte das Vorliegen einer Depression und/oder früher Traumatisierung in dieser Studienkohorte keinen Einfluss auf die kardiovaskuläre Stressreaktivität. Für den prozentualen Anstieg der HF, sBD und dBD in Reaktion auf den TSST konnte kein signifikanter Unterscheid zwischen den Versuchsgruppen gefunden werden.

Obgleich die autonome Dysbalance bei Patienten mit biographisch frühem Stress mittlerweile als möglicher vermittelnder Mechanismus in der Entstehung kardiovaskulärer Erkrankungen anerkannt ist (Spitzer et al. 2016), ist die Studienlage zur kardiovaskulären Stressreaktivität bei dieser Patientengruppe bislang überschaubar. Es gibt bisher nur eine andere Studie, die zur systematischen Aufschlüsselung des Einflusses von ACE und MDD auf die KVR ein komplettes Studiendesign mit den vier Versuchsgruppen ACE, MDD+, 
MDD- und CON durchgeführt hat. Heim und Kollegen (2000) untersuchten 49 Probandinnen mit Depression und/oder Kindheitstraumatisierung bezüglich ihrer KVR. Wie in unserem Versuch wurde an einem ausschließlich weiblichen Patientenkollektiv der TSST als standardisierter Stresstest durchgeführt. Die Arbeitsgruppe konnte im Kontrast zu unserem Ergebnis für die Gruppe MDD+ die höchsten Pulswerte innerhalb der Stressreaktion nachweisen. Im Vergleich zur Kontrollgruppe zeigte sich dieser Unterschied hochsignifikant $(\mathrm{p}<.01)$, ein statistischer Unterschied zu den anderen beiden Versuchsgruppen MDD- und ACE konnte nicht nachgewiesen werden. Die Gruppen MDD- und ACE zeigten im Vergleich zur Kontrollgruppe lediglich eine tendenziell erhöhte Stressreaktivität, sodass dieses Ergebnis je nach Interpretation im Einklang mit unseren Studienergebnissen gesehen werden könnte. Einschränkend muss bei der Studie von Heim und Kollegen (2000) jedoch bedacht werden, dass die Patientenkohorte in diesem Versuch relativ klein war $(\mathrm{n}=49)$, wodurch die statistische Teststärke zu gering gewesen sein könnte, um aussagekräftige Ergebnisse zu liefern (Bortz und Schuster 2010). Zur Überprüfung der KVR wurde in dem Versuch von Heim und Mitarbeitern (2000) lediglich die HF als kardiovaskulärer Marker gemessen; Blutdruckwerte wurden in diesem Studiendesign nicht erfasst. Weitere Studien, welche explizit die Stressreaktivität von depressiven Patienten mit Kindheitstrauma bzw. traumatisierter Patienten ohne Depression untersuchten, existieren in dieser Form bislang noch nicht.

Jedoch gibt es einige Studien, welche die KVR bei frühkindlich traumatisierten Patienten untersuchten. Dabei gilt zu beachten, dass auch in diesen Studien ein Teil der traumatisierten Probandinnen eine komorbide Depression aufwiesen (Otte et al. 2005; Lovallo et al. 2012; Voellmin et al. 2015; Gooding et al. 2016; Meyer et al. 2016), was sowohl den Vergleich mit unserer Versuchsgruppe MDD+ als auch mit der Gruppe ACE erschwert.

Eine aktuelle Studie von Meyer und Kollegen (Meyer et al. 2016) untersuchte die kardiovaskuläre und autonome Stressreaktion bei traumatisierten Frauen mit vorliegender PTBS im Vergleich zu einer gesunden Kontrollgruppe. Zwar wurden in diese Studie nicht ausschließlich Patientinnen mit Kindheitstraumatisierung eingeschlossen, jedoch hatte der deutlich größere Teil der traumatisierten Probandinnen - teilweise neben anderen Missbrauchserfahrungen - lebensgeschichtlich frühen biographischen Stress erlebt (88,5\%). Weiterhin wiesen nahezu alle PTBS-Patientinnen eine komorbide Depression auf (98\%). In dieser Studie wurden zwei psychologische Stresstests durchgeführt (zunächst ,Rechnen', dann ,Babyschreien'). Diese Arbeitsgruppe konnte keinen Unterschied in der Reaktivität der HF bei PTBS-Patientinnen im Vergleich zur Kontrollgruppe auf den Stresstest ,Rechnen' finden, sodass dieses Ergebnis mit unserem vereinbar ist. Jedoch reagierten die PTBS- 
Patientinnen mit einem erniedrigten Pulsanstieg auf den Stressor ,Babyschreien’; zeigten also entgegen unserer Hypothese und den Ergebnissen von Heim und Kollegen (2000) eine erniedrigte KVR. Für die meisten anderen gemessenen kardiovaskulären Parameter (MAP, TPR-I, PEP) wurden in beiden Stresstests keine statistischen Unterschiede in der KVR zwischen PTBS-Patientinnen und den gesunden Kontrollprobandinnen gemessen (Meyer et al. 2016).

Eine negative Korrelation von Kindheitstrauma und der KVR konnte auch in anderen Studien nachgewiesen werden. Gooding und Kollegen (2016) fanden in ihrer Studienkohorte eine geringere Reaktivität von sBD und dBD auf den TSST bei in der Kindheit traumatisierten Probandinnen im Vergleich zu einer nicht-traumatisierten Kontrollgruppe. Sowohl Lovallo und Kollegen (2012) als auch eine Arbeitsgruppe um Voellmin (2015) konnten nachweisen, dass der über psychometrische Tests ermittelte ACE-Score (höhere Werte im ACE-Score reflektieren ein höheres Maß erlebter Kindheitstraumatisierungen) negativ mit dem Pulsanstieg der Probandinnen auf einen psychosozialen Stressor korrelierte. Hierbei muss beachtet werden, dass es sich in allen drei Studien um ein deutlich jüngeres Patientenkollektiv handelt als es in unserer Studie der Fall war und sich die KVR über die Lebensspanne hinweg verändert (siehe Kapitel 1.3.5). Eine Übersicht über das mittlere Alter der Patienten in den hier aufgeführten Studien ist in der Tabelle A2 im Anhang gegeben.

Die Arbeitsgruppe um Otte (2005) konnte eine erhöhte autonome Stressreaktivität bei Probandinnen mit Kindesmisshandlungen nachweisen. Sie fand einen erhöhten Spiegel von 3-Methoxy-4-Hydroxy-Phenoglycol (MHPG) bei Probandinnen mit biographisch früher Traumatisierung im Vergleich zu nicht-traumatisierten Probandinnen. MHPG ist ein Metabolit von Noradrenalin und wird wie die HF und der BD zur Messung der Aktivität des sympathischen Nervensystems angewandt. Die Vergleichbarkeit mit unserer Studie ist jedoch dadurch erschwert, dass ausschließlich Polizeianwärter untersucht wurden und die Studienkohorte zum größten Teil aus männlichen Probanden bestand.

Wie bereits in der Einleitung erwähnt, untersuchten einige Studien die KVR bei depressiven Patienten. Unsere Studienergebnisse stehen für alle gemessenen Parameter HF, sBD und dBD im Einklang mit den Ergebnissen einer Arbeitsgruppe um Taylor (2006), welche keine veränderte KVR bei depressiven Patienten im Vergleich zu mental gesunden Kontrollprobandinnen auf den TSST fand. Dabei handelte es sich allerdings um eine ältere, geschlechterdurchmischte Kohorte; zudem waren die Probanden bereits kardiovaskulär vorerkrankt. Auch Guinjoan und Kollegen (1995) fanden an einer depressiven Kohorte, welche wie in der hier vorliegenden Studie ausschließlich aus Frauen bestand, keinen Un- 
terschied der Versuchsgruppen in der KVR auf einen psychosozialen Stresstest (,Rechnen'). Hamer und Kollegen (2007) führten eine Untersuchung der kardiovaskulären und autonomen Stressreaktivität an einer Kohorte durch, welche anhand der gemessenen Depressionswerte (über die Center for Epidemiological Science Depression Scale (CES-D) gemessen) in eine niedrig- und hoch-depressive Versuchsgruppe eingeteilt wurde. Zur Stressinduktion sollten die Probandinnen über ein Thema sprechen, das sie traurig bzw. wütend macht (,anger-/depressive-Call'). Es wurden verschiedene autonome und kardiovaskuläre Parameter gemessen, wobei die Patientinnen der hoch-depressiven Gruppe lediglich eine stärkere Reaktivität der dBD und des MHPG-Spiegels aufwiesen; der Anstieg der HF, des sBD, des CO und des TPR blieben von depressiven Symptomen unbeeinflusst, sodass dieses Ergebnis zumindest zum Teil mit unserem vereinbar ist.

Schwerdtfeger und Rosenkaimer (2011) führten in ihrem Versuch zwei verschiedene psychosoziale Stresstests durch. Zunächst sollten die Probandinnen eine Rede über ein sozial kontrovers diskutiertes Thema halten und wurden währenddessen gefilmt. Eine verbalinteraktionelle Aufgabe findet sich auch im TSST, welcher in unserem Versuch durchgeführt wurde. Als zweiten Stresstest schauten sich die Probandinnen das Video ihrer Rede selbst an. Obwohl diese Arbeitsgruppe von einer erniedrigten KVR bei depressiven Patienten spricht, zeigt sich beim näheren Betrachten der Daten in dem geschlechterdurchmischten Patientenkollektiv ( $\mathrm{n}=54$, Frauenanteil 68 \%), vergleichbar mit unseren Ergebnissen, kein Unterschied im Anstieg der HF auf einen psychosozialen Stressor bei depressiven Patienten im Vergleich zu einer gesunden Kontrollgruppe. Depressive Patienten in dieser Patientenkohorte zeigten jedoch eine abgeschwächte Reaktivität im dBD und sBD.

Obgleich die Studienlage inhomogen ist, gibt es eine stetig wachsende Ansammlung von Studien, welche Hinweise auf eine gestörte autonome Stressreaktivität bei depressiven Patienten liefert. Entsprechend unserer Hypothese fanden einige Autoren eine erhöhte KVR bei depressiven Patienten. Eine Metastudie $(n=11)$ von Kibler und Ma (2004) fand eine positive Korrelation zwischen Depression und der Reaktivität der HF. Für den sBD und dBD war zwar eine positive Tendenz sichtbar, welche jedoch nicht statistisch abgesichert werden konnte. Auch Matthews und Kollegen (2005) fanden eine positive Korrelation zwischen depressiven Symptomen, gemessen über die Hamilton Anxiety and Depression Scale (HADS), und dem Anstieg der HF sowie auch mit einem erhöhten MAP und TPR in Reaktion auf ein experimentelles Stressereignis.

Die Studienlage ist jedoch inhomogen. So wurde in einigen anderen Studien eine abgeschwächte KVR bei Patienten mit Depression gefunden. De Rooij und Kollegen (2010) führten in dieser Studie an 725 körperlich gesunden Probanden beider Geschlechter ein 
mehrere Aufgaben umfassendes psychologisches Stressexperiment (Stroop-Test, MSST und eine verbal-interaktionistische Aufgabe) durch. Mithilfe der HADS wurden die Patienten in eine depressive Experimental- (HADS-Score $\geq 8$ ) und eine nicht-depressive Kontrollgruppe (HADS-Score $\leq$ 8) eingeteilt. Im Kontrast zu unseren Ergebnissen fand diese Arbeitsgruppe in der linearen Regressionsanalyse eine negative Korrelation zwischen Depressivität und der kardiovaskulären Stressreaktivität; das heißt je höher der Summenscore der HADS war, desto geringer fiel die kardiovaskuläre Stressreaktivität (gemessen über die Marker HF, sBD und dBD) aus.

Einige weitere Studien konnten eine abgeschwächte KVR bei Patienten mit Depression (Carroll et al. 2007; York et al. 2007; Salomon et al. 2009; de Rooij et al. 2010; Schwerdtfeger und Rosenkaimer 2011; Brindle et al. 2013; Yuenyongchaiwat et al. 2017) nachweisen. Eine tabellarische Übersicht über die Ergebnisse vergleichbarer Studien ist in Tabelle 11 gegeben.

Zusammenfassend lässt sich festhalten, dass eine stetig wachsende Zahl von Studien Hinweise auf eine gestörte autonome/kardiovaskuläre Stressregulation bei Patienten mit frühkindlicher Traumatisierung und/oder Depression gibt, wobei in der Literatur sowohl eine erhöhte als auch eine erniedrigte KVR bei den genannten Patientengruppen beschrieben wird. Dies wirft zum einen die Frage auf, warum keiner dieser Effekte in unserer Studie gefunden werden konnte. Zum anderen sollte unter Einbeziehung des aktuellen Forschungsstands in Betracht gezogen werden, dass bei Patientinnen mit Depression und/oder Kindheitstraumatisierung entgegen unserer theoretischen Vorüberlegungen keine erhöhte, sondern eine erniedrigte kardiovaskuläre Stressreaktivität, die den vermittelnden Pfad zur Entwicklung negativer Gesundheitsfolgen darstellen könnte, vorliegt. Diese Aspekte werden in Kapitel 4.3 näher untersucht. 
Tabelle 11: Übersicht über die Ergebnisse der Studien zum Einfluss von Depression und Kindheitstraumata auf die kardiovaskuläre Stressreaktivität

\begin{tabular}{|c|c|c|c|c|c|c|c|}
\hline Studie & $\begin{array}{l}\text { psychi- } \\
\text { scher } \\
\text { Faktor }\end{array}$ & $\begin{array}{l}\text { n } \\
(+ \text { in \%) }\end{array}$ & Stresstool & HF & sBD & dBD & $\begin{array}{l}\text { Weitere } \\
\text { Marker } \\
\text { des ANS }\end{array}$ \\
\hline \multicolumn{8}{|l|}{$\underline{\mathbf{K V R} \uparrow}$} \\
\hline Heim 2000 & $\begin{array}{l}\text { MDD } \\
\text { ACE }\end{array}$ & $\begin{array}{c}49 \\
(100)\end{array}$ & TSST & $\uparrow$ & - & - & - \\
\hline Matthews $2005^{2}$ & ACE & $\begin{array}{c}91 \\
(49)\end{array}$ & MST'T & $\uparrow$ & - & - & $\begin{array}{l}\text { MAP } \uparrow \\
\text { TPR } \uparrow\end{array}$ \\
\hline Otte $2005^{3}$ & ACE & $\begin{array}{c}76 \\
(13,1)\end{array}$ & Video & - & - & - & $\mathrm{MHPG} \uparrow$ \\
\hline Hamer $2007^{4}$ & MDD & $\begin{array}{c}55 \\
(50,1)\end{array}$ & $\begin{array}{l}\text { Vortrag } \\
\text { halten }\end{array}$ & $\leftrightarrow$ & $\leftrightarrow$ & $\uparrow$ & $\begin{array}{c}\mathrm{CO} \leftrightarrow \\
\mathrm{TPR} \leftrightarrow \\
\mathrm{MHPG} \uparrow\end{array}$ \\
\hline$\underline{K V R} \leftrightarrow$ & & & & & & & \\
\hline Guinjoan $1995^{5}$ & MDD & $\begin{array}{l}36 \\
(42)\end{array}$ & Rechenaufgabe & $\leftrightarrow$ & $\leftrightarrow$ & $\leftrightarrow$ & - \\
\hline Heim $2000^{1}$ & $\begin{array}{l}\text { MDD } \\
\text { ACE }\end{array}$ & $\begin{array}{c}49 \\
(100)\end{array}$ & TSST & $\leftrightarrow$ & - & - & - \\
\hline Taylor $2006^{5}$ & MDD & $\begin{array}{c}48 \\
(67)\end{array}$ & TSST & $\leftrightarrow$ & $\leftrightarrow$ & $\leftrightarrow$ & $\begin{array}{c}\mathrm{PEP} \leftrightarrow \\
\mathrm{CO} \leftrightarrow \\
\mathrm{RSA}^{10} \leftrightarrow\end{array}$ \\
\hline Meyer 2016 & PTBS & $\begin{array}{c}92 \\
(100 \%)\end{array}$ & $\begin{array}{l}\text { Rechenaufgabe }^{\mathrm{a}} \\
\text { Babyschreien }^{\mathrm{b}}\end{array}$ & $\begin{array}{l}\leftrightarrow^{\mathrm{a}} \\
\downarrow^{\mathrm{b}}\end{array}$ & & & $\begin{array}{c}\text { MAP } \leftrightarrow \\
\text { BRS } \leftrightarrow^{\mathrm{a}}, \downarrow^{\mathrm{b}} \\
\text { PEP } \leftrightarrow \\
\text { TPR-I } \leftrightarrow\end{array}$ \\
\hline
\end{tabular}

Siehe Fortsetzung Tabelle 11 und Erläuterungen auf der nächsten Seite 
Fortsetzung Tabelle 11: Übersicht über die Ergebnisse der Studien zum Einfluss von Depression und Kindheitstraumata auf die kardiovaskuläre Stressreaktivität

\begin{tabular}{|c|c|c|c|c|c|c|c|}
\hline Studie & $\begin{array}{l}\text { psychi- } \\
\text { scher } \\
\text { Faktor }\end{array}$ & $\begin{array}{c}\mathbf{n} \\
(\uparrow \text { in \%) }\end{array}$ & Stresstool & HF & sBD & sBD & $\begin{array}{l}\text { Andere } \\
\text { Marker des } \\
\text { ANS }\end{array}$ \\
\hline \multicolumn{8}{|l|}{$\underline{\text { KVR } \downarrow}$} \\
\hline Carroll $2007^{7}$ & MDD & $\begin{array}{l}1608 \\
(54 \%)\end{array}$ & PASAT & $\downarrow$ & $\downarrow$ & $\leftrightarrow$ & - \\
\hline Salomon 20095 & MDD & $\begin{array}{l}50 \\
(74 \%)\end{array}$ & Vortrag halten & $\downarrow$ & $\leftrightarrow$ & $\leftrightarrow$ & $\begin{array}{l}\text { PEP } \downarrow \\
\text { CO } \downarrow\end{array}$ \\
\hline de Rooij $2009^{8}$ & MDD & $\begin{array}{l}725 \\
(53 \%)\end{array}$ & $\begin{array}{l}\text { MSTT } \\
\text { Stroop-Test } \\
\text { Vortrag halten }\end{array}$ & $\downarrow$ & $\downarrow$ & $\downarrow$ & - \\
\hline $\begin{array}{l}\text { Schwerdtfeger } \\
2011^{5}\end{array}$ & MDD & $\begin{array}{l}55 \\
(68 \%)\end{array}$ & $\begin{array}{l}\text { Vortrag halten } \\
\text { Video }\end{array}$ & $\leftrightarrow$ & $\downarrow$ & $\downarrow$ & SCR $\downarrow$ \\
\hline Lovallo $2012^{2}$ & $\mathrm{ACE}$ & $\begin{array}{l}426 \\
(58 \%)\end{array}$ & $\begin{array}{l}\text { Vortrag halten } \\
\text { Rechenaufgabe }\end{array}$ & $\downarrow$ & - & - & - \\
\hline Brindle $2013^{5}$ & MDD & $\begin{array}{l}52 \\
(68 \%)\end{array}$ & PASAT & $\downarrow$ & $\downarrow$ & $\leftrightarrow$ & - \\
\hline Gooding $2014^{3}$ & ACE & $\begin{array}{l}145 \\
(58 \%)\end{array}$ & TSST & - & $\downarrow$ & $\downarrow$ & - \\
\hline Voellmin $2015^{2}$ & $\mathrm{ACE}$ & $\begin{array}{l}104 \\
(100 \%)\end{array}$ & MIST & $\downarrow$ & - & - & - \\
\hline $\begin{array}{l}\text { Yuenyongchaiwat } \\
2017^{7}\end{array}$ & MDD & $\begin{array}{l}104 \\
(57 \%)\end{array}$ & $\begin{array}{l}\text { Rechenaufgabe }^{\mathrm{a}} \\
\text { Vortrag halten }^{\mathrm{b}}\end{array}$ & $\begin{array}{l}\downarrow_{\mathrm{a}} \\
\leftrightarrow \leftrightarrow^{\mathrm{b}}\end{array}$ & $\begin{array}{l}\downarrow_{\mathrm{a}} \\
\leftrightarrow \leftrightarrow^{\mathrm{b}}\end{array}$ & $\begin{array}{l}\leftrightarrow^{\mathrm{a}} \\
\leftrightarrow^{\mathrm{b}}\end{array}$ & $\mathrm{CO}^{\mathrm{a}}, \leftrightarrow^{\mathrm{b}}$ \\
\hline
\end{tabular}

Einteilung der Ergebnisse vergleichbarer Studien in KVR $\uparrow$ (Patientengruppe zeigte erböbte KVR), $\leftrightarrow$ (Patientengruppe zeigte keine veränderte KVR) und $\downarrow$ (Patientengruppe zeigte erniedrigte KVR), wobei die Zuordnung aufgrund mehrerer gemessener Parameter nicht immer ganz eindeutig ist. Bei Studien, in welchen mehrere psychosoziale Stresstests durchgeführt wurden, werden unterschiedliche Ergebnisse in den jeweiligen Stresstests explizit gekennzeichnet. Einteilung in die Versuchsgruppen wird folgendermaßen gekennzeichnet: ${ }^{1} M D D+$ vs. MDD- vs. ACE vs. CON, ${ }^{2}$ Einteilung der Patientinnen anhand der ,number of ACE' im ETI (0,1,2-3, 24) brw. eigenen Kriterien in low-medium und high-adversity group (Lovallo 2012) ${ }^{3}$ ACE vs. CON, ${ }^{4}$ Anhand des CES-D Einteilung in hoch-depressive Versuchsgruppe (CES-D-Score 2 16) vs. niedrig-depressive Versuchsgruppe (CES-D-Score S16), ${ }^{5}$ MDD vs. CON, ${ }^{6}$ PTBS vs. CON (in der Studie von Meyer (2016) wurde zusätzlich noch zwischen PTBS-Patientinnen mit und obne herzwirksamer Medikation unterschieden. In dieser Tabelle werden nur die Ergebnisse dargestellt, die zwischen dem gesamten PTBS-Kollektiv und der CON eindeutig sind), ${ }^{7}$ Keine explizite Einteilung in Versuchsgruppen: Hier wurden die in den Depressionsfragebögen ermittelten Scores in der linearen Regressionsanalyse als Kovariable miteinbezogen, ${ }^{8}$ Anhand der HADS Einteilung in hoch-depressive Versuchsgruppe (HADS-Score $\geq 8$ ) vs. niedrigdepressive Versuchsgruppe (HADS-Score $\leq 8)$. KVR: kardiovaskuläre Stressreaktion, TSST: Trier Social Stress Test, MSTT Mirror Star Tracing Task, PASAT: Paced Auditory Serial Addition Test, MIST: Montreal Imaging Stress Task, 
HF: Hersfrequen₹, sBD: systolischer Blutdruck, dBD: diastolischer Blutdruck, MAP: mittlerer arterieller Druck, TPR(-I): totaler peripherer Widerstand(-Index), MHPG: 3-Methoxy-4-Hydroxyphenoglykol, CO: Herzzeitvolumen, PEP: Präejektionszeit, RSA: respiratorische Sinusarrbythmie, BRS: Baroreflexsensibilität, SCR: elektrodermale Aktivität

\subsubsection{Hypothese: Depression, Kindheitstrauma und kardiovaskuläre Recovery}

Abweichend von unserer zweiten Hypothese konnte in unserem Versuch bei Probandinnen mit Depression und/oder frühkindlicher Traumatisierung im Vergleich zur gesunden Kontrollgruppe keine prolongierte Stressreaktion im Sinne einer verzögerten Recovery 1 und 2 der kardiovaskulären Marker HF, sBD und dBD nach dem psychosozialen Stressereignis nachgewiesen werden.

In einigen der oben zitierten Studien, welche die Stressreaktivität von depressiven bzw. frühkindlich traumatisierten Patienten untersuchten, wurden auch Messungen der kardiovaskulären Parameter nach einer Erholungsphase durchgeführt, welche auf das Stressereignis folgte (Light et al. 1998; Heim et al. 2000; Taylor et al. 2006; Hamer et al. 2007; Salomon et al. 2009; de Rooij et al. 2010; Brindle et al. 2013; Voellmin et al. 2015). In mehreren Studien wurden diese Messwerte jedoch nicht weiter analysiert, sodass diese Studien nicht zum Vergleich mit unseren Ergebnissen der Recovery herangezogen werden können (Heim et al. 2000; Taylor et al. 2006; Brindle et al. 2013; Voellmin et al. 2015). In zwei der Studien werden in der Auswertung zwar die Recovery-Werte erwähnt, es wurde jedoch keine ausreichende Operationalisierung für dieses Konstrukt durchgeführt, sodass keine eindeutige Interpretation der Ergebnisse möglich ist (Light et al. 1998; Taylor et al. 2006). In den übrigen Studien variiert die Operationalisierung der Recovery. Um unsere Arbeit besser in den aktuellen Literaturkontext einordnen zu können, haben wir zwei gängige Formen der Operationalisierungen der Recovery einbezogen (siehe Kapitel 1.3.3 und 2.4.1.1) und Recovery 1 sowie Recovery 2 definiert. Die Recovery 1 entspricht dem Vergleich der gemessenen kardiovaskulären Parameter der Stressreaktivität mit den Werten nach der Erholungsphase. Eine vergleichbare Vorgehensweise lässt ist auch in einer Studie von de Rooij und Kollegen (2010) zu finden, welche oben bereits detailliert besprochen wurde. Im Kontrast zu unseren Ergebnissen fand diese Arbeitsgruppe eine negative Korrelation zwischen dem Summenscore der HADS und der Recovery 1 für die Marker sBD, dBD und HR. Unserer Hypothese entsprechend führte Depressivität in dieser Kohorte zu einer verzögerten Recovery der kardiovaskulären Marker HF, sBD und dBD. Eine Analyse der Recovery 1 bei Patientinnen mit PTBS im Vergleich zu einer gesunden Kontrollgruppe wurde von Meyer und Kollegen (2016) durchgeführt. Es zeigten sich keine unterschiedlichen Recovery-Werte der HF, MAP, TPR-I und PEP. Allerdings fiel auf, dass PTBS- 
Patientinnen eine geringere Barorezeptorsensibilität (BRS) aufwiesen. Dieser Befund wird in dem hierauf folgenden Kapitel detailliert besprochen.

Als Recovery 2 definierten wir den Vergleich der Differenz der gemessenen Werte nach der Erholungsphase mit denen der Baseline (siehe Kapitel 2.4.1.1). Eine statistische Analyse der Recovery 2 wird auch in vergleichbaren Studien von Hamer und Kollegen (2007), Schwerdtfeger und Rosenkaimer (2011) sowie Salomon und Mitarbeitern (2009, 2013) durchgeführt.

Hamer und Kollegen (2007) konnten in ihrem oben bereits ausführlicher dargestellten Versuch feststellen, dass der dBD sowie auch der MHPG-Spiegel bei Probandinnen der hochdepressiven Versuchsgruppe nach einer 30-minütigen Erholungsphase im Vergleich zur Baseline signifikant erhöht war, während sich diese Parameter bei niedrig-depressiven Probandinnen schon wieder vollständig erholt hatten, was im Einklang mit unseren formulierten Erwartungen steht. Wie in unserem Versuch zeigte sich die Recovery der kardiovaskulären Marker HF und sBD (sowie auch für die Parameter CO und TPR) unbeeinflusst von depressiven Symptomen.

Wie in Kapitel 4.1.1 schon erwähnt wurde, führten Schwerdtfeger und Rosenkaimer (2011) in ihrem Versuch zwei verschiedene psychosoziale Stresstests (,Vortrag halten', ,Video') durch; an diese Stressoren schloss sich jeweils eine Erholungsphase an, wobei die Operationalisierung der Recovery der hier definierten Recovery 2 entspricht. Nach dem Stresstest ,Vortrag halten' konnten in der Recovery 2 keine Unterschiede zwischen depressiven Probandinnen und der Kontrollgruppe gefunden werden, was im Einklang mit unseren Studienergebnissen steht. Wider Erwarten zeigten depressive Patienten in der Recovery 2 nach der Durchführung des passiven Stresstests, Video' signifikant geringere Werte als die Vergleichsgruppe in der Recovery 2, was einer beschleunigten Recovery entspricht (siehe Kapitel 1.3.3 und 2.4.1.1).

Salomon und Kollegen (2009) widmeten sich in ihrer Studie explizit der Auswertung der Recovery und liefern sehr exakte Informationen über die Definition und Operationalisierung des Konstrukts. Diese Arbeitsgruppe konnte nach der Durchführung eines verbalen Stresstests, analog zu unseren Ergebnissen, keine veränderte Recovery 2 des Blutdrucks (sBD und DPB) bei depressiven Patienten im Vergleich zur Kontrollgruppe nachweisen. Im Kontrast zu unseren Studienergebnissen zeigten depressive Probandinnen dieser Kohorte jedoch eine verzögerte Erholung der HF.

Eine Übersicht über vergleichbare Studien und deren Ergebnisse zur kardiovaskulären Recovery findet sich in Tabelle 12. 
Zusammenfassend lässt sich Folgendes festhalten: Obgleich einige Studien Hinweise darauf geben, dass nicht nur eine dysregulierte kardiovaskuläre Stressreaktivität, sondern auch eine gestörte Recovery langfristig ein möglicher vermittelnder Mechanismus zur Entwicklung kardiovaskulärer Erkrankungen sein könnte (Schuler und O’Brien 1997; Steptoe und Marmot 2006; Chida und Steptoe 2010), widmen sich bisher nur wenige Studien explizit diesem Thema. Zudem unterliegt das Konstrukt der Recovery in der Literatur keiner einheitlichen Operationalisierung, was die Vergleichbarkeit erschwert. Eine intensivere Beschäftigung mit der Recovery in Studien zur kardiovaskulären Stressreaktion bei traumatisierten und depressiven Patienten wäre für weitere Studien wünschenswert. In den oben zitierten Studien zur Recovery bei traumatisierten und depressiven Probandinnen zeigte sich ein uneinheitliches Bild. Während manche Autoren keine veränderte Recovery bei den Patientengruppen nachweisen konnten, gibt es gleichfalls Hinweise auf eine gestörte Recovery. Entgegen den formulierten Erwartungen zeigten aktuelle Studien neben einer prolongierten teilweise auch eine ,schnellere' Recovery, also eine verkürzte Stressreaktion bei depressiven Patienten (Hamer et al. 2007; Schwerdtfeger und Rosenkaimer 2011). Diese Ergebnisse stützen die Überlegung, dass möglicherweise nicht (nur) eine erhöhte und prolongierte, sondern auch eine erniedrigte und verkürzte Stressreaktion Ausdruck einer autonomen Dysbalance sein könnte. Dieser Aspekt soll in Kapitel 4.3 detailliert diskutiert werden. 
Tabelle 12: Übersicht über Ergebnisse der Studien zum Einfluss von Depression und Kindheitstraumata auf die kardiovaskuläre Recovery

\begin{tabular}{|c|c|c|c|c|c|c|c|}
\hline Studie & $\begin{array}{l}\text { Psychischer } \\
\text { Faktor }\end{array}$ & $\begin{array}{c}\text { n } \\
(+ \text { in \%) }\end{array}$ & Stresstool & HF & sBD & dBD & $\begin{array}{c}\text { Andere } \\
\text { Marker } \\
\text { des } \\
\text { ANS }\end{array}$ \\
\hline KVRec 1 & & & & & & & \\
\hline de Rooij 20091 & MDD & $\begin{array}{l}725 \\
(53 \%)\end{array}$ & $\begin{array}{l}\text { MSTT } \\
\text { Stroop-Test } \\
\text { Vortrag halten }\end{array}$ & $\downarrow$ & $\downarrow$ & $\downarrow$ & - \\
\hline Meyer 2016 2 & PTBS & $92(100 \%)$ & $\begin{array}{l}\text { Rechnen, } \\
\text { Babyschreien }\end{array}$ & $\leftrightarrow$ & - & - & $\begin{array}{l}\text { MAP } \leftrightarrow \\
\text { BRS } \uparrow \\
\mathrm{PEP} \leftrightarrow \\
\text { TPR-I }\end{array}$ \\
\hline$\underline{\text { KVRec } 2}$ & & & & & & & \\
\hline Hamer $2007^{3}$ & MDD & $\begin{array}{l}55 \\
(50,1 \%)\end{array}$ & Vortrag halten & $\leftrightarrow$ & $\leftrightarrow$ & $\uparrow$ & $\begin{array}{l}\mathrm{CO} \leftrightarrow \\
\mathrm{TPR} \leftrightarrow \\
\mathrm{MHPG} \uparrow\end{array}$ \\
\hline $\begin{array}{l}\text { Schwerdtfeger } \\
2011^{4}\end{array}$ & MDD & $\begin{array}{l}55 \\
(68 \%)\end{array}$ & $\begin{array}{l}\text { Rede }^{\mathrm{a}} \\
\text { Video }^{\mathrm{b}}\end{array}$ & $\begin{array}{l}\leftrightarrow^{\mathrm{a}} \\
\leftrightarrow^{\mathrm{b}}\end{array}$ & $\begin{array}{l}\leftrightarrow^{a} \\
\uparrow_{b}\end{array}$ & $\begin{array}{l}\leftrightarrow^{a} \\
\uparrow_{b}\end{array}$ & - \\
\hline $\begin{array}{l}\text { Salomon } 2009^{4} \text {, } \\
2013^{4}\end{array}$ & MDD & $\begin{array}{l}50 \\
(74 \%)\end{array}$ & Vortrag halten & $\downarrow$ & $\leftrightarrow$ & $\leftrightarrow$ & $\begin{array}{l}\text { TPR } \\
\mathrm{PEP} \leftrightarrow \\
\mathrm{CO} \leftrightarrow\end{array}$ \\
\hline
\end{tabular}

Einteilung der Ergebnisse vergleichbarer Studien in KV Rec 1 und 2. Bei Studien, in welchen mehrere psychosoziale Stresstests durchgeführt wurden, werden unterschiedliche Ergebnisse der jeweiligen Stresstests explizit gekennzeichnet. Einteilung in die Versuchsgruppen wird folgerndermaßen gekennzeichnet: ${ }^{1}$ Anhand der HADS Einteilung in hoch-depressive Versuchsgruppe (HADS-Score 28) vs. niedrig-depressive Versuchsgruppe (HADS-Score $\leq 8),{ }^{2}$ PTBS vs. CON (in der Studie von Meyer (2016) wurde zusätzlich noch zwischen PTBS-Patientinnen mit und obne herzwirksamer Medikation unterschieden. In dieser Tabelle werden nur die Ergebnisse dargestellt, die zwischen dem gesamten PTBS-Kollektiv und der CON eindeutig sind), ${ }^{3}$ Anhand des CES-D Einteilung in hoch-depressive Versuchsgruppe (CES-D-Score $\left.\geq 16\right)$ vs. niedrig-depressive Versuchsgruppe (CES-D-Score S16), ${ }^{4}$ MDD vs. CON. MDD: major depressive disorder, PTSB: posttraumatische Belastungsstörung, MSST:Mirror Star Tracing Task, KVRec:kardiovaskuläre Recovery, KVRec $\downarrow$ : verzögerte Recovery, KVRec T: beschleunigte Recovery, HF: Hersfrequenz, sBD: systolischer Blutdruck, dBD: diastolischer Blutdruck, MAP: mittlerer arterieller Druck, TPR(-I): totaler peripherer Widerstand(-Index), MHPG: 3-Methoxy-4-Hydroxyphenoglykol, CO: Herzzeitvolumen, PEP: Präejektionszeit, RSA: respiratorische Sinusarrbythmie, BRS: Barorezeptorsensibilität, 


\subsection{Methodenkritische Betrachtungen}

Im folgenden Kapitel werden unter Einbeziehung des aktuellen Forschungsstands Gründe für unsere insignifikanten Studienergebnisse erörtert. Hierfür werden die Versuchsverfahren sowie die Patientenkohorte näher beleuchtet. Dabei soll diskutiert werden, weshalb trotz der gefundenen Nulleffekte die Alternativhypothesen zutreffen könnten, sowie Möglichkeiten für die Richtigkeit der Annahme der Nullhypothesen aufgezeigt werden.

\subsubsection{Stressinduktion und Erholung}

\subsubsection{TSST}

Zur Stressinduktion wurde in unserem Versuch der TSST angewandt. Dieser psychosoziale Stresstest gilt als anerkanntes Verfahren zur experimentellen Stressinduktion. In mehreren Studien konnte eine Aktivierung der SNA (Gold et al. 2004; Jezova et al. 2004; Het et al. 2009) bzw. des kardiovaskulären Systems (Childs et al. 2006; Rimmele et al. 2007) durch den TSST nachgewiesen werden. Giles und Kollegen (2014) sowie eine Arbeitsgruppe um Skoluda (2015) untersuchten zudem den Einfluss des TSST im Vergleich zu anderen experimentalen Stressoren auf die kardiovaskuläre Reaktivität an gesunden Probandinnen. Beide Arbeitsgruppen konnten nachweisen, dass der TSST einen signifikanten Anstieg des Pulses auslöste. Im Vergleich zu anderen experimentellen Induktoren von psychosozialem Stress, wie dem socialevaluativen Kaltwasser-Stresstest (engl.: socially evaluative cold pressor test, kurz: SECPT), dem Stroop-Test sowie dem Lösen von Rechenaufgaben, bewirkte der TSST in beiden Studien sogar die am stärksten gemessene kardiovaskuläre Stressreaktivität.

Auch in der hier beschriebenen Kohorte führte der TSST im Gesamtkollektiv erwartungsgemäß zu einer signifikanten Erhöhung des sBD, dBD und der HF. Bei Betrachtung der einzelnen Versuchsgruppen konnte dieser Effekt jedoch nicht allseits wiedergefunden werden. So konnte bei traumatisierten Probandinnen ohne Depression in keinem der gemessenen Parameter eine signifikante kardiovaskuläre Stressreaktivität nachgewiesen werden (siehe Kapitel 3.2.1).

Zunächst muss in Betracht gezogen werden, dass diese insignifikanten Ergebnisse möglicherweise durch die statistischen Verfahren bedingt sind. Zur Vermeidung des Fehlers 1. Art beim multiplen Testen innerhalb der hier untersuchten Stichprobe wurde eine Anpassung des $\alpha$-Niveaus mittels Bonferroni-Holm durchgeführt. Dieses Verfahren gilt in der Statistik als vergleichsweise wenig konservativ. Dennoch steigt dadurch die Wahrscheinlichkeit, dass die Alternativhypothese abgelehnt wird, obwohl sie wahr ist (Fehler 2. Art). In der hier vorliegenden Arbeit bedeutet dies konkret, dass ein möglicherweise bestehender signifikanter Anstieg bzw. Abfall der kardiovaskulären Parameter nicht detektiert werden konnte. 
Zudem gilt: Je größer ein Stichprobenumfang, desto wahrscheinlicher kann ein bestehender Effekt erkannt werden (Bortz und Schuster 2010). Für das Gesamtkollektiv ( $n=143$ ) konnte für alle gemessenen Parameter ein signifikanter Anstieg in Reaktion auf den TSST und ein signifikanter Abfall nach der Erholungsphase gemessen werden. Die GruppengröBen in unserer Kohorte schwankten zwischen 22 und 52 Versuchspersonen, wobei die Gruppe ACE die kleinste Versuchsgruppe darstellte und somit in der statistischen Analyse die geringste Teststärke aufwies (Bortz und Schuster 2010). Dies liefert eine mögliche Erklärung dafür, weshalb die Gruppe ACE in keinem der gemessenen Parameter einen signifikanten Anstieg der Parameter aufwies. Für eine optimale statistische Analyse wären gleich große Versuchsgruppen wünschenswert gewesen, dies ist für zukünftige Studien anzustreben.

Bei Betrachtung der Ergebnisse stellt sich dennoch die Frage, ob der TSST ein geeignetes Instrument zur kardiovaskulären Stressinduktion bei traumatisierten Probandinnen darstellt. In drei vergleichbaren Studien wurde der TSST bei traumatisierten Probandinnen angewandt (Heim et al. 2000; Lovallo et al. 2012; Gooding et al. 2016). Unser Ergebnis steht im Kontrast zu den gefundenen Effekten von Heim und Kollegen (2000). In diesem Versuch konnte sowohl bei Gesunden als auch bei Patientinnen mit Kindheitstraumatisierung ein signifikanter Anstieg der HF auf den TSST verzeichnet werden. In den Versuchen von Lovallo und Kollegen (2012) und der Arbeitsgruppe um Gooding (2016) wurden zwar Vergleiche der kardiovaskulären Stressreaktivität anhand der HF (Lovallo 2012) sowie des sBD und des dBD (Gooding 2016) zwischen den Versuchsgruppen untereinander durchgeführt, es jedoch wurde nicht überprüft, ob es tatsächlich zu einer signifikanten Stressreaktivität gekommen war, sodass diesbezüglich keine Vergleichswerte vorliegen. Es wurde somit bisher in keiner vergleichbaren Studie überprüft, ob der TSST tatsächlich zu einem signifikanten Anstieg des sBD und dBD bei traumatisierten Probanden führt, sodass die hier vorliegenden Ergebnisse in weiteren Studien überprüft werden sollten.

\subsubsection{Erholungsphase}

Im Gesamtkollektiv zeigte sich nach der Erholungsphase ein signifikanter Abfall aller drei Messparameter (Recovery 1). Im Vergleich zur Baseline waren der sBD und der dBD nach der Erholungsphase in der Gesamtheit noch erhöht, die Herzfrequenz hatte sich schon wieder dem Ausgangswert angenähert. Bei Betrachtung der einzelnen Versuchsgruppen konnten diese Effekte nicht allseits wiedergefunden werden (siehe Kapitel 3.2.1). Im Vergleich der Versuchsgruppen untereinander konnten keine signifikanten Unterschiede in der kardiovaskulären Recovery 1 und 2 gefunden werden (siehe Kapitel 3.2.2.2). 
Nachdem der TSST durchgeführt worden war, füllten unsere Patientinnen während der Erholungsphase Fragebögen zum subjektiv erlebten Stress aus. Dieses Vorgehen ist vergleichbar mit der Studie von De Rooij und Kollegen (2010). In einer anderen Studie durften die Patienten lesen (Hamer et al. 2007). In weiteren Studien saßen oder lagen die Patienten während der Erholungsphase in einer ruhigen Umgebung (Salomon et al. 2009), teilweise wurden ihnen zusätzlich, entspannend wirkende' Videos bzw. Musik vorgespielt (Schwerdtfeger und Rosenkaimer 2011; Salomon et al. 2013; Meyer et al. 2016). Es ist denkbar, dass das Ausfüllen der Fragebögen und somit die weiteren, - wenn auch nur mentalen - Aufgaben, die kardiovaskulären Parameter beeinflusst und somit auch einen Effekt auf die Recovery-Werte gehabt haben könnten.

Diese Überlegung wird von den Ergebnissen der Studie von Fredrickson und Levenson (1998) gestützt. Die Autoren konnten an gesunden Probanden zeigen, dass positive im Vergleich zu traurigen Emotionen zu einer schnelleren kardiovaskulären Recovery nach einem psychischen Stressereignis führen. Des Weiteren sollte bedacht werden, dass Depressionen häufig mit einer übermäßigen Grübelneigung (engl.: rumination) einhergehen (Nolen-Hoeksema 2000; Spasojevic und Alloy 2001; Balsamo 2010). Nach der ,perservative cognition therory' wird davon ausgegangen, dass das Festhalten an negativen Gedanken im Sinne von sorgenreichem oder ärgerlichem Grübeln zu einer erhöhten und prolongierten kardiovaskulären Stressreaktion und langfristig zur Entstehung kardiovaskulärer Erkrankungen führt (Brosschot et al. 2006). Eine Arbeitsgruppe um Busch (2017) untersuchte in einer großen Metastudie (43 Studien mit insgesamt 3.348 Teilnehmern) den moderierenden Einfluss von sorgenreichem und ärgerlichem Grübeln bei depressiven Patienten auf die Stressreaktion (gemessen über HF, sBD und dBD). Die Ergebnisse geben Hinweise darauf, dass möglicherweise nicht die Depression per se, sondern das begleitende Grübeln den vermittelnden Weg zwischen Depression und einer veränderten kardiovaskulären Stressreaktion darstellt (Busch et al. 2017). Dieser Effekt konnte für alle drei erfassten kardiovaskulären Marker sBD, dBD und HF gefunden werden (Busch et al. 2017).

In weiteren Studien sollte dieser Aspekt berücksichtigt und die Auswirkungen der verschiedenen Umgebungsbedingungen während der Erholungsphase auf die kardiovaskuläre Recovery bei depressiven und traumatisierten Probanden untersucht werden.

\subsubsection{Messverfahren}

\subsubsection{Messgeräte, Messzeiten und Operationalisierung}

Eine Ursache für die - teilweise - insignifikanten Ergebnisse der Stressreaktivität und Recovery sowie des Gruppenvergleichs könnte in der Wahl der Messzeitpunkte und -geräte 
begründet liegen. Zur Erfassung der hier ausgewerteten Parameter HF, sBD und dBD wurde ein halbautomatisches Blutdruckmessgerät verwendet und zu verschiedenen Messzeitpunkten während des Versuchs gemessen.

Einzelne Messungen von Blutdruck- und Pulswerten entsprechen einer Momentaufnahme und könnten dadurch störanfällig sein. In den meisten anderen Studien zur kardiovaskulären Stressreaktivität wurden kontinuierliche, nicht-invasive Messungen von Blutdruck und Puls durchgeführt (Taylor et al. 2006; Hamer et al. 2007; de Rooij et al. 2010; Schwerdtfeger und Rosenkaimer 2011; Gooding et al. 2016; Meyer et al. 2016). Dieses Verfahren ermöglicht eine exaktere Messung der kardiovaskulären Parameter während des gesamten Versuchsablaufs. Statt einzeln erfasster Werte können durch die kontinuierliche Messung Mittelwerte für die verschiedenen Messintervalle des Versuchsablaufs berechnet werden, was den Verlauf von BD und HF während des gesamten Versuchs abbildet und somit weniger anfällig für Fehler sein könnte. Schwerdtfeger und Rosenkaimer (2011) sowie eine Arbeitsgruppe um Voellmin (2015) konnten durch dieses Messverfahren signifikante Gruppenunterschiede detektieren. Zudem können durch die kontinuierliche Messung maximale und minimale Werte der kardiovaskulären Marker der einzelnen Phasen ermittelt werden. De Rooij und Kollegen (2010) verwendeten zur Messung der Stressreaktivität und Berechnung der Recovery maximale Werte der kardiovaskulären Marker in den jeweiligen Messintervallen und kamen somit beim Vergleich der Versuchsgruppen zu signifikanten Studienergebnissen.

In unserem Versuch wurde die Stressreaktivität operationalisiert über die Differenz der kardiovaskulären Messwerte vor (t2) und direkt nach dem Stresstest (t3) (siehe Kapitel 2.4.1.1). In vergleichbaren Studien, in denen eine nicht-kontinuierliche Messung der kardiovaskulären Parameter durchgeführt wurde, gab es mindestens einen weiteren Messzeitpunkt während des TSST, der zur Messung des Anstiegs der kardiovaskulären Messparameter diente (Heim et al. 2000; Giles et al. 2014; Skoluda et al. 2015). Alle genannten Autoren konnten zeigen, dass ein signifikanter Anstieg der Herzfrequenz während des TSST messbar war. In den Versuchen von Giles und Mitarbeitern (2014) sowie Skoluda und Kollegen (2014) konnte nachgewiesen werden, dass die Herzfrequenz zum Messzeitpunkt direkt nach dem TSST sogar schon wieder auf das Ausgangniveau abgefallen war.

Heim und Mitarbeiter (2000) führten in ihrem Versuch gemäß diesen Befunden einen Vergleich der Stressreaktivität zwischen den Versuchsgruppen ACE, MDD+, MDD- und CON durch. Sie konnten nachweisen, dass die Gruppe MDD+ einen signifikant höheren Pulsanstieg als die Gruppe CON aufwies. Dieser Unterschied konnte jedoch nur für den Messzeitpunkt während des TSST belegt werden, nach dem TSST hatten sich auch hier die 
Parameter bereits wieder in Richtung der Baseline normalisiert und ein signifikanter Gruppenunterschied ließ sich nicht mehr nachweisen.

Die hier genannten Aspekte legen nahe, dass die kardiovaskuläre Recovery - speziell der HF - bereits während des TSST stattfindet und nicht erst im Anschluss an den Stresstest. Unter diesem Gesichtspunkt scheint die Konstruktvalidität durch die Operationalisierung der Stressreaktivität und Recovery in dem hier vorliegenden Versuch fraglich und liefert eine denkbare Erklärung für die gefundenen Nulleffekte. Möglicherweise wäre eine exakte Detektion der Stressreaktivität und Recovery sowie von signifikanten Gruppenunterschieden durch einen weiteren Messzeitpunkt bzw. eine kontinuierliche Messung der kardiovaskulären Parameter während des TSST auch in unserer Kohorte möglich gewesen. Eine Anpassung der Messmethode und Operationalisierung dahingehend wäre für zukünftige Studien wünschenswert.

Des Weiteren sollte bedacht werden, dass das experimentelle Setting per se einen Stressfaktor dargestellt haben könnte. Die Patientinnen durchliefen nach ihrer Ankunft eine Ruhephase, um das kardiovaskuläre Stressniveau zu Beginn des TSST so gering wie möglich zu halten. Jedoch lagen die kardiovaskulären Werte durch das experimentelle Setting als Stressfaktor möglicherweise bereits zu Beginn des Versuchs über ihrem natürlichen Ruheniveau, sodass sie in Reaktion auf den eigentlichen Stresstest nicht signifikant anstiegen.

\subsubsection{Messparameter}

Es gibt einige Parameter, die zur Messung des autonomen Nervensystems in der aktuellen Forschung Anwendung finden (siehe Kapitel 1.3.3). Diese Arbeit konzentrierte sich auf die Auswertung der kardiovaskulären Marker sBD, dBD und HF. In vergleichbaren Studien wurden teilweise zusätzliche Parameter zur Erfassung der kardiovaskulären und autonomen Stressreaktivität und Recovery herangezogen, wie z. B. PEP, TPR, CO und SCR (siehe Kapitel 4.2). Es ist denkbar, dass eine Ausweitung dieser Studie auf die Erfassung weiterer Parameter zur Aufdeckung von möglicherweise bestehenden Gruppenunterschieden hätte beitragen können.

Zudem geben Herrmann-Lingen und Kollegen (2010) zu bedenken, dass die autonome Stressreaktion oft zu allgemein betrachtet wird und schlagen vor, eine Beurteilung auf verschiedenen Ebenen vorzunehmen. So sollten zum einem die verschiedenen sympathischen Mechanismen detailliert aufgeschlüsselt werden und sowohl vorwiegend $\alpha$-adrenerg (z. B. TPR-I, BD) bzw. $\beta$-adrenerg gesteuerte Parameter (z. B. CI, HF, PEP) erfasst werden. Zum anderen sollten auch beide Arme des ANS, das SNS und das PNS innerhalb der Stressreaktion betrachtet werden (Herrmann-Lingen et al. 2010). Hierzu führten Meyer und 
Mitarbeiter (2016) die bereits oben zitierte Studie zur Stressreaktivität bei Frauen mit komplexen Traumafolgestörungen durch. Neben Markern der sympathischen Aktivität (u. a. MAP, HR, PEP, TPR-I) wurde zur Aufschlüsslung des vagalen Einflusses auf die Stressregulation eine Erfassung der BRS durchgeführt. Dieser Messparameter gibt Auskunft über die sympathovagale Balance: Bei einer Dominanz des Sympathikotonus sinkt die BRS ab, bei Überwiegen des vagalen Tonus steigt sie an (La Rovere et al. 1995; 2008). Während Probandinnen der CON auf den emotionalen Stressor ,Babyschreien’ mit einer Erhöhung des BRS reagierten, welche sich auch in der Recovery-Phase stabil hielt, konnte dieser Effekt bei Patientinnen mit PTBS nicht gemessen werden. Die Autoren schlussfolgern, dass eine gestörte vagale Aktivität bei den PTBS-Patientinnen die physiologischen Konsequenzen einer generell gestörten autonomen Stressantwort auf einen emotionalen Stressor darstellt (Meyer et al. 2016). Weitere Studien sollten daher auch besonderes Augenmerk auf das sympathovagale Zusammenspiel in der Stressregulation richten.

Die bisher aufgeführten Messvariablen sollen die autonome Aktivität indirekt über die Messung von Organfunktionen erfassen. Es muss jedoch berücksichtigt werden, dass die verschiedenen Organsysteme sehr komplex sind und möglicherweise nicht ausschließlich über das ANS gesteuert werden. Ein Beispiel dafür ist der Blutdruck, welcher in der Stressreaktion zwar maßgeblich über Katecholamine gesteuert, jedoch auch durch weitere vasokonstriktive Substanzen beeinflusst wird. So werden innerhalb der Stressreaktion auch die synergistisch wirkenden Hormone $\mathrm{CRH}$ und $\mathrm{ADH}$ ausgeschüttet. $\mathrm{ADH}$ bewirkt eine Vasokonstriktion und eine Erhöhung des Blutvolumens durch eine verminderte Flüssigkeitsausscheidung über die Niere und erhöht somit den BD. CRH stimuliert die Ausschüttung von Cortisol, welches über Mineralkortikoidrezeptoren vasokonstriktiv wirkt (Ehlert 2011). Zudem erhöht Cortisol die Potenz vasokonstriktiver Substanzen im Sinne eines permissiven Effekts und wirkt sich somit steigernd auf den BD aus (Paschke 2014). Das wirft die Frage auf, ob die Messung der autonomen Aktivität auch auf höherer Ebene, also noch vor ihrer Beeinflussung der Organe, gemessen werden kann, um somit die Einflüsse anderer Regulationssysteme, wie der HHNA, auf die gemessene Variable gering zu halten. Eine Möglichkeit könnte die direkte Messung der Katecholaminspiegel oder ihrer Metaboliten darstellen. Wie bereits oben beschrieben untersuchte die Arbeitsgruppe um Otte (2005) die Aktivität des ANS von traumatisierten Patienten während eines Stressereignisses über 3Methoxy-4-Hydroxy-Phenoglycol (MHPG), einem Metabolit von ADR und NA im Blut, und konnte eine erhöhte autonome Stressreaktivität bei traumatisierten Personen nachweisen. Dies wäre ein Ansatz für weitere Studien. 
Zudem muss bedacht werden, dass die verschiedenen Stresssysteme sehr vielschichtig und komplex sind. Die Entschlüsselung der Interaktion zwischen dem ANS und der HHNA gewinnt in der neueren Forschung immer mehr an Aufmerksamkeit (Koch et al. 2017). Einige Studien widmeten sich dabei dieser Wechselbeziehung auf neuroanatomischer und physiologischer Ebene. So ist bereits bekannt, dass CRH-Releasing-Neuronen im Nucleus paraventricularis des Hypothalamus und NE-Releasing-Neuronen im Hirnstamm über Fasern anatomisch miteinander verbunden sind (Ulrich-Lai und Herman 2009). Neurophysiologisch gibt es Hinweise, dass NA die Ausschüttung von CRH aus dem Nucleus paraventricularis im Hypothalamus potenziert und somit zur Aktivität der HHNA beiträgt (Pacak et al. 1995; Chrousos 2009). Wiederum führt die Applikation von CRH in die Neuronen des Locus coeruleus, der größten Ansammlung noradrenerger Nervenzellen im ZNS, zu einer erhöhten Aktivität dieser Neuronen (Chrousos und Gold 1992). Zudem gibt es Erkenntnisse, dass die Synthese von ADR aus NA im Nebennierenmark hauptsächlich durch den parakrinen Effekt von Cortisol stimuliert wird (Paschke 2014).

Die Arbeitsgruppe um Andrews (2012; 2013) machte sich zum Ziel, die wechselseitige Beziehung der HHNA und SNA innerhalb der Stressreaktion aufzuschlüsseln und entwickelte dazu das kombinierte Dexamethason/TSST- und Propranolol/TSST-Paradigma. In einer ersten Studie wurden 30 gesunde männliche Probanden untersucht. An der Hälfte der Probanden wurde ein Dexamethason-Supressionstest (DST) durchgeführt (DEX-Gruppe), die andere Versuchsgruppe blieb unbehandelt (PLC-Gruppe). Beim DST wird über die Gabe von Dexamethason eine negative Feedback-Reaktion ausgelöst und die CRH- und ACTH-Ausschüttung unterdrückt, also die HHNA blockiert. Anschließend durchliefen alle Probanden den TSST zur Stressinduktion. Diese Studie konnte bei der DEX- im Vergleich zur PLC-Gruppe, neben der Suppression der Cortisolausschüttung, signifikant höhere Werte der HF sowohl während des TSST als auch in der Recovery-Periode nachweisen (alle $\mathrm{p}<.05$ ). Diese Ergebnisse legen nahe, dass eine fehlende Cortisolantwort zu einer stärkeren Aktivierung der SNA führt, was möglicherweise die Konsequenz einer erhöhten Ausschüttung von CRH darstellt (Andrews et al. 2012).

In einem zweiten Versuch wurde bei sonst gleichem Versuchsaufbau bei der Experimentalgruppe (PROP-Gruppe) über die Gabe von Propranolol die Antwort der SNA blockiert. Propranolol ist ein nicht-selektiver B-Antagonist und hemmt die Aktivität von ADR und NA. Die PROP-Gruppe zeigte im Vergleich zur PLC-Gruppe einen deutlich höheren Anstieg des Cortisolspiegels in Reaktion auf den TSST. Die Autoren konnten nachweisen, dass auf die Suppression der SNA eine erhöhte Cortisolantwort folgt. Auf dem zentralen Level bedeutet dies, dass ein Fehlen der physiologischen SNA-Antwort registriert wird und 
zu einer stärkeren Aktivierung der HHNA führt (Andrews und Pruessner 2013). Zusammenfassend lässt sich festhalten, dass der Wegfall eines der beiden Stresssysteme (z. B. durch eine experimentell induzierte Blockade) zu einer stärkeren Reaktion des anderen Systems führt - vermutlich im Sinne einer Kompensation. Diese Ergebnisse stützen die Annahme, dass keines der Stresssysteme als einzelne Einheit betrachtet werden kann, sondern vielmehr ein Zusammenspiel der verschiedenen Systeme und Reaktionen im Organismus zur Aufrechterhaltung der Homöostase beiträgt. Die hier angeführten Studienergebnisse der Arbeitsgruppe um Andrews (2012; 2013) gelten dabei nur für gesunde, männliche Probanden mit vermutlich - normalerweise - intakten Stressregulationssystemen. Bisher gibt es noch keine Studien, die vergleichbare Untersuchungen an depressiven und/oder traumatisierten Probandinnen durchgeführt haben. In dem hier vorliegenden Versuch konnte keine veränderte KVR und KVRec bei Patientinnen mit Kindheitstraumatisierung und/oder Depression gefunden werden. Unter Berücksichtigung des aktuellen Forschungsstands führt dies jedoch nicht zwingend zu dem Schluss, dass bei den hier untersuchten Patientinnen ein stabiles autonomes System vorlag. Bestehende Imbalancen des ANS wurden möglicherweise über eine Kompensation durch die HHNA kaschiert und waren somit über die kardiovaskuläre Stressreaktivität und Recovery nicht messbar.

Wie in der Einleitung bereits beschrieben, strebt der Körper laut dem Konzept der Allostase stets einen ausgeglichenen Zustand an (Sterling und Eyer 1988; McEwen 1998). Bei chronischer Belastung kommt es durch Verschleiß dieser Systeme zu einer allostatischen Last, welche sich in einem inadäquaten Antwortverhalten auf Stress äußert und langfristig durch eine Überlastung zu kardiovaskulären Erkrankungen und anderen pathologischen Folgen führen kann (Seeman et al. 1997; McEwen 1998). Die hier untersuchten Patientinnen waren im Schnitt 34,9 Jahre alt. Zur Ausbildung kardiovaskulärer Folgeerscheinungen war unsere Kohorte möglicherweise noch zu jung. Es ist nicht auszuschließen, dass potentiell bestehende Imbalancen der Stresssysteme im jüngeren Alter noch ausreichend gegenreguliert werden können, sodass keine körperlichen Folgen zu messen sind. Diese Annahme wird von der Erkenntnis gestützt, dass besonders bei Frauen das Manifestationsalter kardiovaskulärer Erkrankungen recht hoch liegt, die Inzidenz ab dem 75. Lebensjahr sogar exponentiell ansteigt und dadurch insgesamt sogar höher ist als bei Männern (Ladwig et al. 2013). Die Arbeitsgruppe um Ladwig und Kollegen (2013) konstatiert, dass Studien mit Probandinnen in jüngeren Lebensabschnitten deshalb nicht ausreichen, um körperliche Effekte messen zu können und dadurch häufig unterschätzt werden. Es wäre interessant, die hier beschriebene Kohorte zu einem späteren Zeitpunkt erneut zu untersuchen, um denkbare zukünftige körperliche Folgen bestehender autonomer Dysbalancen zu detektie- 
ren und gegebenenfalls mit der Entwicklung von kardiovaskulären Erkrankungen in Verbindung bringen zu können. Dabei sollte besonderes Augenmerk auf die Interaktion zwischen der SNA und der HHNA gelegt werden.

\subsubsection{Studienkohorte, soziodemographische und klinische Betrachtungen}

Für unsere Arbeit konnten wir 143 Studienteilnehmerinnen gewinnen. Damit ist die Kohorte größer als in vielen anderen vergleichbaren Studien (Heim et al. 2000; Salomon et al. 2009; Schwerdtfeger und Rosenkaimer 2011). Da mit der Größe des Stichprobenumfangs die Teststärke ansteigt (Bortz und Schuster 2010), stellt dies einen Vorteil unserer Studie dar.

Ein Großteil der Probandinnen in der hier vorliegenden Studie wurde über öffentliche Annoncen und Aushänge rekrutiert. Zusätzlich wurden depressive Patientinnen der Versuchsgruppen mit einer führend depressiven Erkrankung über die spezialisierten Stationen der an dieser Studie beteiligten psychiatrisch-psychotherapeutischen Kliniken gewonnen. Wie Spitzer und Kollegen (2018) bereits kritisch zu einer von unserer nur geringfügig erweiterten Stichprobe konstatierten, erhielten stationär aufgenommene Patientinnen für ihre Teilnahme an dem Versuch keinen finanziellen Ausgleich, wohingegen öffentlich rekrutierte Probandinnen eine Aufwandsentschädigung von 200 Euro bekamen. Dies stellt eine Schwäche unserer Studie dar, da dieses Vorgehen möglicherweise zu systematischen Schätzfehlern geführt haben könnte.

Weitere Quellen von Verzerrungen sind insbesondere Geschlecht, Alter und sozioökonomischer Status der Versuchspersonen (Weiß 2013), weshalb sie hier besondere Beachtung erfahren sollen.

In unsere Studie wurden ausschließlich Frauen untersucht. Da einige Studien einen Geschlechtsunterschied in der kardiovaskulären Stressreaktivität bestätigen konnten (siehe Kapitel 1.3.5), können unsere Ergebnisse nicht uneingeschränkt generalisiert werden. Die alleinige Untersuchung von Probandinnen ist gleichwohl auch eine Stärke der Studie, da wir so das Geschlecht als möglichen Störfaktor ausschließen konnten. Es gibt bisher nur wenige Studien auf diesem Gebiet, die diesen möglichen Confounder gänzlich eliminiert haben (Guinjoan et al. 1995; Heim et al. 2000; Voellmin et al. 2015; Meyer et al. 2016). Da es bisher kaum Studien gibt, welche sich ausschließlich auf Frauen beziehen, wurde für diese Arbeit jedoch auch auf Literatur zurückgegriffen, welche Patientenkollektive beider Geschlechter betrachtet. Es wurde bei der Auswahl der Literatur darauf geachtet, dass diese Studien das Geschlecht in ihren statistischen Analysen berücksichtigten. Eine Übersicht über den Frauenanteil der hier analysierten Studien ist in Tabelle 11, Kapitel 4.2.1 gegeben. 
Das mittlere Alter unserer Patientinnen lag bei 34,9 Jahren, wobei sich die vier Versuchsgruppen diesbezüglich nicht voneinander unterschieden. Auch in den Studien von Heim (2000), Salomon (2009) und Yuenyongchaiwat (2017) wurde eine ,mittelalte' Kohorte betrachtet. Einige Studien untersuchten jedoch ein deutlich jüngeres (Hamer et al. 2007; Lovallo et al. 2012; Brindle et al. 2013; Voellmin et al. 2015; Gooding et al. 2016) bzw. älteres Studienkollektiv (Taylor et al. 2006; York et al. 2007; de Rooij et al. 2010).

Unsere vier Versuchsgruppen wiesen hinsichtlich der soziodemographischen und klinischen Merkmale Schuljahre, Rauchen, Größe, Gewicht und BMI keine signifikanten Gruppenunterschiede auf. Bezüglich des Schulabschlusses zeigte sich eine leichte statistische Tendenz zu Gruppenunterschieden $(\mathrm{p}=.06$ ), wobei die Probandinnen der Kontrollgruppe die höchsten Schulabschlüsse aufwiesen. Die Probandinnen der Gruppe ACE befanden sich in dieser Studienkohorte hinsichtlich des Schulabschlusses im Mittelfeld, gefolgt von den Versuchsteilnehmerinnen der Versuchsgruppen mit einer führend depressiven Erkrankung (MDD+ und MDD-), welche die niedrigsten Schulabschlüsse auswiesen. Ein verglichen mit der Allgemeinbevölkerung niedrigerer sozioökonomischer Status ist bei depressiven Personen ein häufig begleitender Umstand (Busch et al. 2013; Robert Koch-Institut 2015), sodass dieses Ergebnis nicht weiter überrascht.

Ein möglicher, in dieser Studie nicht erfasster Confounder ist der Menstruationszyklus. Wie in Kapitel 1.3.5 bereits beschrieben wurde, können hormonelle Schwankungen während des weiblichen Menstruationszyklus eine Auswirkung auf die KVR haben, sodass eine fehlende Erfassung des Merkmals und seine Parallelisierung über die Versuchsgruppen dahingehend eine potentielle Fehlerquelle darstellt und somit zu Schlussfolgerungen führt, deren Reproduzierbarkeit in weiteren Studien überprüft werden muss. Dies stellt eine Limitation unserer Studie dar.

Zudem wurden Probandinnen mit einem BMI >30 (ab Adipositas Grad I) von der Studie ausgeschlossen. Laut aktueller Forschungsergebnisse besteht eine positive Korrelation zwischen Depression bzw. frühkindlicher Traumatisierung und Übergewicht (Felitti et al. 1998; Faith et al. 2002; McElroy et al. 2004; Bentley und Widom 2009; Chartier et al. 2009; Gilbert et al. 2009), sodass durch unser Ausschlusskriterium adipöse Probandinnen in den Experimentalgruppen unterrepräsentiert sein könnten. Obgleich die Studienlage inhomogen ist, gibt es Hinweise auf einen positiven Zusammenhang zwischen Adipositas und einer gestörten KVR (Singh und Shen 2013; Krishnaveni et al. 2018). Möglicherweise haben wir also das Übergewicht als entscheidende Mediatorvariable aus unserer Studie eliminiert, was ein Grund für die gefundenen Nulleffekte sein könnte. 
Nicht an unserer Studie teilnehmen konnten Probandinnen, die innerhalb der letzten Woche psychotrope Medikamente wie Neuroleptika, Benzodiazepine oder Opiate eingenommen hatten. Unser Patientenkollektiv war allerdings nicht komplett unmediziert, etwa ein Drittel der Versuchsteilnehmerinnen nahm ein orales Kontrazeptivum ein. Mehrere Probandinnen bekamen L-Thyroxin, sowie zwei Patientinnen Betablocker. Bezüglich der Einnahme somatischer Medikamente wiesen die Versuchsgruppen keine statistischen Unterschiede auf. Einige Patientinnen der Gruppen mit einer führend depressiven Erkrankung nahmen Psychopharmaka ein (MDD-: 54,3 \%, MDD+: 37,5 \%). Dies kann möglicherweise zu Ergebnisverzerrungen führen, da einige kardiovaskulär wirksame Medikamente die Stressreaktivität verändern können (de Rooij et al. 2010; Meyer et al. 2016). Ein ausreichend großes und gleichzeitig komplett unmediziertes Patientenkollektiv stellte im Rahmen dieser Studie eine nicht lösbare Herausforderung dar. Nur zwei der oben zitierten Studien betrachteten ein Patientenkollektiv, das frei von jeglicher Medikamenteneinnahme war (Hamer et al. 2007; Schwerdtfeger und Rosenkaimer 2011). Diese Studien umfassten allerdings lediglich 54 bzw. 55 Probanden.

Da bei Patientinnen mit manifester PTBS eine gestörte Stressreaktivität vorliegt (Zaba et al. 2015) und die Studienergebnisse dadurch verzerrt werden könnten, wurden Patienten mit dieser Störung von unserer Studie ausgeschlossen. Mehrere Probandinnen, fast ausschließlich aus den Versuchsgruppen mit einer führend depressiven Erkrankung, wiesen jedoch psychiatrische Komorbiditäten auf, wie z. B. somatoforme Störungen, Zwangsstörungen und anhaltende affektive Störungen. Wie schon Bornschein (2014) in ihrer Dissertation zur Stressreaktivität bei Frauen mit komplexen Traumafolgestörungen in Bezug auf ihre Patientenkohorte feststellte, wäre gleichwohl in unserem Fall eine medikamentenfreie Patientenkohorte ohne weitere Begleitdiagnosen zur reinen Erfassung des Einflusses von Depressionen und/oder Kindheitstraumatisierungen auf die Stressreaktion optimal gewesen. Auch in unserer Kohorte bestehend aus depressiven und/oder frühkindlich traumatisierten Patienten sind psychiatrische Komorbiditäten sowie die Einnahme von Psychopharmaka ein bekanntes Phänomen und entsprechen somit einem Abbild der klinischen Realität (siehe Kapitel 1.2).

Zusammenfassend lässt sich festhalten, dass die hier analysierten Versuchsgruppen wie angestrebt überwiegend nach den wichtigsten soziodemographischen Parametern parallelisiert wurden. Zwar haben einige Merkmale möglicherweise einen Effekt auf die KVR; laut Weiß (2013) ist eine Verzerrung durch potentielle Confounder jedoch vor allem zu befürchten, wenn sich die Gruppen a priori in den wesentlichen Charakteristika unterscheiden. 


\subsubsection{Depression und Kindheitstraumatisierung}

Um den Einfluss von Depression und Kindheitstraumatisierungen auf die kardiovaskuläre Stressreaktion systematisch zu untersuchen, wurden unsere Probandinnen in vier verschiedene Versuchsgruppen eingeteilt: MDD+, ACE, MDD- und CON. Diese Gruppeneinteilung wurde bisher nur in einer anderen vergleichbaren Studie von Heim und Mitarbeitern (2000) vorgenommen. Andere Autoren betrachteten lediglich isoliert den Aspekt der Depression (Carroll et al. 2007; York et al. 2007; Salomon et al. 2009; de Rooij et al. 2010; Brindle et al. 2013) bzw. der Kindheitstraumatisierung (Lovallo et al. 2012; Voellmin et al. 2015; Gooding et al. 2016) und ihren Einfluss auf die KVR.

Zur Überprüfung unserer Gruppeneinteilung für depressive und traumatisierte Patientinnen kamen mehrere psychometrische Instrumente zum Einsatz. Anhand des BDI und der MADRS wurden unsere Probandinnen auf eine bestehende Depression untersucht. Die Teilnehmerinnen der Gruppen mit einer führend depressiven Erkrankung wiesen im Mittel signifikant höhere Scores im BDI und in der MADRS auf als Probandinnen der Versuchsgruppen ohne Depression. Zur Einschätzung der biographisch frühen Traumatisierungen wurden sowohl der CTQ als Fragebogen zur Selbsteinschätzung sowie auch ein halbstrukturiertes Interview, das ETI, durchgeführt. Wie erwartet wiesen die Patientinnen der Gruppen mit frühkindlicher Traumatisierung in den Gesamtscores beider Tests signifikant höhere Werte auf als Patientinnen aus den Versuchsgruppen ohne frühkindliche Traumatisierung. Die Gruppen ACE und MDD+ unterschieden sich in ihren Summenscores nicht. Dies war das von uns erwartete Ergebnis, sodass diese Gruppenzuteilung die von uns angestrebte Güte erfüllte.

$\mathrm{Ob}$ retrospektive Instrumente zur Erfassung frühkindlicher Traumatisierung zu einer Erinnerungsverzerrung führen und je nach Stimmungslage eine Verharmlosung oder Aggravierung des erlebten Geschehens mit sich ziehen könnten, ist Gegenstand aktueller Forschung. Eine Studie von Hardt und Kollegen (2010) konnte jedoch beim Vergleich retrospektiver und longitudinaler prospektiver Erfassung früher Traumatisierung keine unterschiedlichen Testergebnisse nachweisen. Dennoch gilt es zu beachten, dass Traumata, die pränatal oder im Säuglingsalter auftreten, bei der retrospektiven Erfassung aufgrund fehlender Erinnerung unterschätzt werden könnten. Dies ist insofern als besonders kritisch zu betrachten, als dass neuere Forschungsergebnisse darauf hinweisen, dass speziell Traumatisierungen in der prä- und postnatalen Periode einen größeren Einfluss auf die Stressreaktivität haben könnten als Misshandlungen in späteren Abschnitten der Kindheit (Bosch et al. 2012). Um dennoch mögliche Informationsbias so gering wie möglich zu halten und die erlebte Traumatisierung so exakt wie möglich zu erfassen, wurde auf den Einsatz einer 
breiten Diagnostik und validierter Instrumente zur Erfassung von Kindesmisshandlung geachtet, was eine Stärke unserer Studie darstellt. Beide angewandten Verfahren stellen gängige Instrumente zur Erfassung frühkindlicher Traumatisierung dar und wurden auch in weiteren vergleichbaren Studien verwendet. Eine Übersicht über die verwendeten psychometrischen Tests in vergleichbaren Studien liefert die Tabelle A2 im Anhang.

\subsubsection{Generelle Diskussion und Implikationen}

Da sich die soziodemographischen Merkmale weitestgehend gleichmäßig über die Versuchsgruppen verteilten, wurden sie als nicht-verzerrende Störgrößen behandelt und in den weiteren statistischen Analysen nicht berücksichtigt. Zur Überprüfung unserer Hypothesen wurden einfaktorielle Varianzanalysen durchgeführt, welche sich zum Vergleich der Versuchsgruppen in der Ausprägung eines bestimmten Faktors (hier kardiovaskuläre Stressreaktivität bzw. Recovery) eignen. Ein Einfluss der Merkmale Depression und/oder Kindheitstraumatisierung auf die kardiovaskuläre Stressreaktivität und Recovery konnte in dieser Kohorte nicht gefunden werden. Wie bereits in Kapitel 3.2.1 detailliert beschrieben wurde, zeigte sich jedoch in der hier untersuchten Kohorte eine auffallend große Standardabweichung der Messparameter. Dies reflektiert eine sehr inhomogene Stressantwort des Gesamtkollektivs und sowie auch innerhalb der einzelnen Versuchsgruppen. So reagierte ein nicht zu vernachlässigender Teil der Patientinnen nicht mit einem Anstieg, sondern gar mit einem Abfall der kardiovaskulären Parameter auf den TSST. Ein entsprechend heterogenes Bild zeigte sich auch für die Recovery 1 und 2 (siehe Tabelle A1 im Anhang).

Entsprechend McEwens Theorie der Allostase und allostatischen Last (1998) könnte nicht nur eine erhöhte und prolongierte, sondern auch eine unzureichende Reaktion der Stresssysteme ('Typ III: ,inadequate response', vgl. Kapitel 1.3.1.3) langfristig zu einer Dysregulation und zu negativen Gesundheitsfolgen führen. Mehrere longitudinale Studien fanden einen Zusammenhang zwischen einer erniedrigten Stressreaktion und der Entstehung von kardiovaskulären Erkrankungen (Carroll et al. 2009; Lovallo 2012; Phillips et al. 2013). Da die untersuchten Merkmale Kindheitstrauma und/oder Depression in unserer Kohorte keinen Einfluss auf die Stressreaktivität und Recovery hatten, stellt sich die Frage, welche Merkmale stattdessen zu dieser breiten Fächerung der kardiovaskulären Stressreaktionen führten.

Lovallo und Kollegen (2011) widmen sich dieser Fragestellung. Zur statistischen Auswertung weiterer Studien zur kardiovaskulären Stressreaktivität schlagen sie folgendes Vorgehen vor: Zunächst sollten die Ergebnisse beider Enden der Verteilung, also sowohl extrem hohe als auch extrem niedrige Messwerte, klar gegenüber ,der Mitte', welche der normati- 
ven homöostatischen Stressreaktion entspricht, abgegrenzt werden. Folgende Punkte sollten analysiert werden: Welche Charakteristika kennzeichnen die Personen, die eine extrem hohe bzw. extrem niedrige Stressreaktion aufweisen? Es wird vorgeschlagen, eine multivariate Analyse zur Identifikation der Zusammenhänge zwischen soziodemographischen sowie psychologischen Merkmalen und der kardiovaskulären Stressreaktion durchzuführen. Es sollte untersucht werden, welche Charakteristiken sich im unteren und oberen Extrem finden und inwiefern sie sich von der normativen Mitte unterscheiden. Anschließend sollte evaluiert werden, zu welchen protektiven bzw. gesundheitsschädlichen Auswirkungen es in den Extremgruppen kommt, da bei Probanden mit einer erniedrigten Stressreaktivität möglicherweise andere Mechanismen zur Entwicklung kardiovaskulärer Erkrankungen beitragen als bei Probanden mit einer erhöhten Stressreaktivität (Lovallo 2011).

Dabei sollte bedacht werden, dass die Dysregulation des ANS und der HHNA als physiologische Veränderungen nur einen möglichen vermittelnden Faktor in der Entstehung kardiovaskulärer Erkrankungen bei depressiven und traumatisierten Patienten darstellen. Ein weiterer, bislang wenig erforschter Ansatz führt über das Immunsystem und seine Rolle innerhalb der Stressreaktion. In einer aktuellen Studie von Chiang und Kollegen (2017) konnte über die Messung von Interleukin-6 ein erhöhtes inflammatorisches Geschehen in Reaktion auf den TSST bei depressiven und traumatisierten Patienten nachgewiesen werden, wobei es Hinweise darauf gibt, dass eine verstärkte Entzündungsreaktion langfristig zur Ausbildung kardiovaskulärer Erkrankungen führen könnte (Fu 1995; Pearson et al. 2003). Wie in der Einleitung bereits beschrieben, werden verschiedene Mechanismen diskutiert, über welche sich chronisch psychosozialer Stress negativ auf das HerzKreislaufsystem auswirken kann (von Känel 2011; Spitzer et al. 2013). Neben einer veränderten Stressreaktivität werden weitere physiologische Veränderungen (z. B. das metabolische Syndrom), ein schädliches Gesundheitsverhalten (z. B. Bewegungsmangel, Rauchen, ungünstige Ernährung), und psychische Veränderungen (z. B. Feindseligkeit, Persönlichkeitsveränderungen) in Betracht gezogen. Die genauen vermittelnden Prozesse bei depressiven und traumatisierten Patienten in der Entstehung kardiovaskulärer Erkrankungen sind jedoch bisher weitestgehend ungeklärt, sodass hier noch weiterer Forschungsbedarf besteht.

\subsection{Schlussfolgerungen und Ausblick}

Da die autonome Dysbalance einen möglichen vermittelnden Mechanismus zwischen chronisch psychosozialem Stress und der Entwicklung kardiovaskulärer Erkrankungen darstellt, ist die wissenschaftliche Untersuchung der kardiovaskulären Stressreaktion von 
besonderem Interesse. Nur unter Kenntnis der vermittelnden Pfade kann bei diesen Patienten eine gezielte Prävention stattfinden.

In unserem Versuch zeigten traumatisierte Probandinnen ohne Depression in keinem der drei gemessenen Parameter eine kardiovaskuläre Stressreaktivität in Reaktion auf den TSST. Da zu diesem Phänomen bisher wenige Vergleichsstudien existieren, sollte die Replizierbarkeit dieses Ergebnisses untersucht werden und damit auch, ob der TSST bei diesen Patienten ein zuverlässiges Mittel zur experimentellen Stressinduktion darstellt.

Zudem sollte zur Optimierung der Konstruktvalidität eine Anpassung der Messmethode erfolgen. Dabei sollte mindestens eine weitere Messung der kardiovaskulären Parameter während des Stressereignisses stattfinden, optimalerweise sogar eine kontinuierliche Messung derselben. Dadurch könnten die kardiovaskuläre Stressreaktivität und Recovery sowie potentielle Gruppenunterschiede sensibler detektiert werden.

In zukünftigen Studien sollte das Spektrum der Messparameter erweitert werden. Dabei sollten zur differentiellen Betrachtung der KVR und KVRec Variablen erfasst werden, welche einen Rückschluss auf das sympathovagale Zusammenspiel innerhalb der Stressreaktion zulassen, wobei sich hierfür z. B. die BRS und HRV eignen.

$\mathrm{Da}$ in unserer Studienkohorte ein autonomes Hyperarousal möglicherweise über einen Ausgleich durch die HHNA kaschiert wurde, sollten weitere Studien die Interaktion zwischen dem ANS und der HHNA bei traumatisierten und/oder depressiven Patientinnen untersuchen. Ein möglicher Ansatz stellt dabei die Anwendung des kombinierten Dexamethason/TSST- und Propranolol/TSST- Paradigma (Andrews et al. 2012; Andrews und Pruessner 2013) bei diesen Patientengruppen dar. Dies würde einen wichtigen Beitrag zum Verständnis der Grundprinzipien der Stressregulation über das Zusammenspiel der verschiedenen Stresssysteme ANS und HHNA bei traumatisierten und depressiven Personen leisten.

Während die Messung der KVR bei traumatisierten und/oder depressiven Patienten bereits Eingang in die Forschung gefunden hat, wurde die Untersuchung der KVRec eher vernachlässigt. Wünschenswert wären weitere Studien zur KVRec, wobei eine einheitliche und klare Definition dieses Konstruktes zur besseren Interpretation und Vergleichbarkeit dringend notwendig ist.

Mit dem in dieser Arbeit durchgeführten Querschnittsdesign konnte nur eine Momentaufnahme der KVR und KVRec gemacht werden. Zudem war unsere Studienkohorte noch relativ jung. Um die langfristigen körperliche Folgen chronisch psychosozialen Stresses zu messen, sollten deshalb longitudinale Studien durchgeführt werden. 
Entgegen der klassischen Sicht, dass eine erhöhte und prolongierte Stressreaktion langfristig zu negativen Gesundheitsfolgen führt, sollte unter Einbeziehung neuerer Forschungserkenntnisse in Betracht gezogen werden, dass möglicherweise (auch) eine erniedrigte und verkürzte Stressreaktion zur Entwicklung kardiovaskulärer Folgeerscheinungen beitragen könnte.

Zur Beantwortung dieser Fragestellung wird in Anlehnung an Lovallo (2011) für weitere Studien zur kardiovaskulären Stressreaktivität und Recovery folgendes Vorgehen in der Auswertung der erhobenen Daten vorgeschlagen:

- klare Abgrenzung der Ergebnisse beider Enden der Verteilung, also sowohl extrem hohe als auch extrem niedrige Messwerte, gegenüber ,der Mitte', welche der normativen homöostatischen Stressreaktion entspricht

- Durchführung einer multiplen Strukturanalyse (z. B. eines linearen Regressionsmodells, siehe u. a. de Rooij et al. 2010) zur Identifikation der Zusammenhänge zwischen soziodemographischen sowie psychologischen Merkmalen und der kardiovaskulären Stressreaktivität und Recovery

- Beantwortung der Frage: Wie unterscheiden sich die Charakteristiken der Probanden aus beiden Extremen von den Personen der ,Mitte’?

- Da möglicherweise bei den jeweiligen Extremgruppen unterschiedliche Charakteristiken dominieren, sollten protektive bzw. gesundheitsschädliche Faktoren von Personen mit einer erniedrigten (und/oder verkürzten) kardiovaskulären Stressreaktion und einer erhöhten (und/oder prolongierten) Stressreaktion ermittelt und miteinander verglichen werden. Die Kenntnis dieser Merkmale könnte Auskunft über die vermittelnden Pfade zur Entwicklung negativer Gesundheitsfolgen in den jeweiligen Extremgruppen geben. 


\section{$5 \quad$ Zusammenfassung}

Hintergrund: Die Pathogenese von KVE im Allgemeinen und der aHTN im Speziellen ist nur über multifaktorielle Wege erklärbar, wobei chronisch psychosozialem Stress (u. a. ACE und MDD) eine bedeutende Rolle zugesprochen wird. Dabei können sich psychosoziale Determinanten über direkte pathophysiologische Mechanismen, wie einer Dysregulation des ANS, negativ auf das Herz-Kreislaufsystem auswirken. Da das ANS die kardiovaskuläre Stressreaktion maßgeblich beeinflusst, könnte entsprechend McEwens Theorie der Allostase und allostatischen Last eine erhöhte und prolongierte Stressreaktion auf einen akuten psychosozialen Stressor langfristig zur Entwicklung einer aHTN führen. In mehreren longitudinalen Studien konnte dieser Zusammenhang bereits bestätigt werden. Die Studienlage zur kardiovaskulären Stressreaktivität und- recovery bei Patienten mit frühkindlicher Traumatisierung und Depression ist inhomogen. Ziel dieser Studie ist es, systematisch den Einfluss von Depression und/oder Kindheitstraumatisierung auf die kardiovaskuläre Stressreaktivität und Recovery bei Frauen aufzuschlüsseln.

Methoden: Insgesamt wurden 143 Probandinnen in die Studie eingeschlossen und anhand psychometrischer Interviews und Fragebögen in vier Versuchsgruppen eingeteilt: ACE (gesunde Probandinnen mit frühen Traumatisierungen, $\mathrm{n}=22$ ), MDD+ (depressive Probandinnen mit Kindheitstraumatisierung, $\mathrm{n}=32$ ), MDD- (depressive Probandinnen ohne Kindheitstraumatisierung, $\mathrm{n}=52$ ) und $\mathrm{CON}$ (gesunde Kontrollgruppe ohne Kindheitstraumatisierung, $\mathrm{n}=37$ ). Zur experimentellen Stressinduktion wurde ein psychosozialer Stresstest, der TSST, durchgeführt. Systolische und diastolische Blutdruckwerte sowie Pulswerte wurden vor dem TSST (t2), direkt nach dem TSST (t3) und nach einer zehnminütigen Erholungsphase (t4) erfasst.

Ergebnisse: Die vier Versuchsgruppen zeigten bezüglich der meisten erhobenen soziodemographischen Daten keine statistischen Unterschiede. Probandinnen der CON wiesen im Vergleich zu den Patientengruppen eine statistische Tendenz zu einer höheren Schulbildung auf. Aus den Patientengruppen wiesen mehrere Probandinnen psychische Komorbiditäten auf und einige Patientinnen mit einer führend depressiven Erkrankung (MDD- und $\mathrm{MDD}+)$ nahmen Psychopharmaka ein.

Im Gesamtkollektiv konnte in Reaktion auf den TSST ein signifikanter Anstieg aller drei gemessenen kardiovaskulärer Parameter verzeichnet werden. Bei Betrachtung der einzelnen Versuchsgruppen war dieses Bild jedoch inhomogen: Die Versuchsgruppe ACE zeigte in keinem der Messparamater einen signifikanten Anstieg; zudem konnte eine statistisch rele- 
vante Erhöhung des Pulses lediglich für die Versuchsgruppe MDD+ verzeichnet werden. Im Kontrast zu unseren Erwartungen zeigten sich keine signifikanten Unterschiede in der KVR zwischen den vier Versuchsgruppen, sodass die erste Hypothese abgelehnt werden muss.

Nach der Erholungsphase wies das Gesamtkollektiv einen signifikanten Abfall aller kardiovaskulären Parameter auf (KVRec 1). Im Vergleich zur Baseline waren der sBD und der dBD nach der Erholungsphase noch signifikant erhöht, der Puls war bereits wieder auf das Ausgangsniveau abgesunken (KVRec 2). Auch in der KVREc 1 und KVRec 2 zeigte sich dieses Bild bei Betrachtung der Reaktionen innerhalb der einzelnen Versuchsgruppen inhomogen. Entgegen unserer Vermutung konnte kein statistischer Gruppenunterschied in der KVRec 1 und 2 gefunden werden, sodass auch die zweite Hypothese verworfen werden muss.

Auffallend war eine sehr große Standardabweichung über alle Versuchsgruppen und Messzeitpunkte hinweg.

Konklusionen: In dieser Studienkohorte führte das Vorliegen einer MDD und/oder ACE im Vergleich zu gesunden Kontrollprobandinnen weder zu einer erhöhten KVR noch zu einer verzögerten KVRec. Dennoch sollten zukünftige Studien diesen Zusammenhang weiterhin unter Anpassung des Studiendesigns überprüfen. Zur Optimierung der Konstruktvalidität sollte ein weiterer Messzeitpunkt bzw. eine kontinuierliche Messung der kardiovaskulären Parameter während des Versuchs stattfinden. Zudem sollte das Spektrum der Messparameter erweitert werden und eine Differenzierung des sympathovagalen Zusammenspiels vorgenommen werden. Von Interesse ist auch die Interaktion zwischen dem ANS und der HHNA.

In Bezug auf die statistischen Methoden sollten in weiteren Studien multivariate Analysen zur Identifikation des Zusammenspiels psychischer und soziodemographischer Merkmale und der kardiovaskulären Stressreaktivität/Recovery durchgeführt werden. 


\section{Abbildungsverzeichnis}

Abbildung 1: Mechanismen, die über eine veränderte kardiovaskuläre Stressreaktion den Zusammenhang zwischen chronisch psychosozialem Stress und der Entstehung kardiovaskulärer Erkrankungen vermitteln könnten

Abbildung 2: Darstellung des Versuchsablaufs .................................................................................................. 21

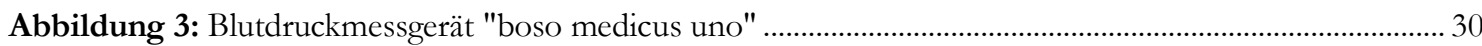

Abbildung 4: Operationalisierung der Stressreaktivität und Recovery ............................................................... 32

Abbildung 5: Versuchsablauf, Stressreaktivität und Recovery ….......................................................................... 33

Abbildung 6: Übersicht über die ermittelten Nebendiagnosen für die Versuchsgruppen mit einer führend depressiven Erkrankung.....

Abbildung 7: Übersicht über eingenommene psychopharmakologische Substanzgruppen für die Versuchsgruppen mit einer führend depressiven Erkrankung

Abbildung 8: Ergebnisse des ETI- und CTQ-Summenscores der vier Versuchsgruppen

Abbildung 9: Ergebnisse des BDI- und MADRS-Summenscores der vier Versuchsgruppen

Abbildung 10: Verlauf der systolischen Blutdruckwerte der einzelnen Versuchsgruppen über t2, t3 und t4 49

Abbildung 11: Verlauf der diastolischen Blutdruckwerte der einzelnen Versuchsgruppen über t2, t3 und t4 50 Abbildung 12: Verlauf der Herzfrequenz der einzelnen Versuchsgruppen über t $2, \mathrm{t} 3$ und $t 4$...

Abbildung 13: Darstellung der Stressreaktivität als prozentuale Veränderung des diastolischen und systolischen Blutdrucks sowie der Herzfrequenz von t2 $\rightarrow$ t3 im TSST nach Gruppenzugehörigkeit

Abbildung 14: Darstellung der Recovery 1 als prozentuale Veränderung des systolischen Blutdrucks, des diastolischen Blutdrucks und der Herzfrequenz von $\mathrm{t} 3 \rightarrow \mathrm{t} 4$ im TSST nach Gruppenzugehörigkeit...... 55

Abbildung 15: Darstellung der Recovery 2 als prozentuale Veränderung des systolischen Blutdrucks, des diastolischen Blutdrucks und der Herzfrequenz von $\mathrm{t} 2 \rightarrow \mathrm{t} 4$ im TSST nach Gruppenzugehörigkeit 


\section{Tabellenverzeichnis}

Tabelle 1: Übersicht über die vier Versuchsgruppen........................................................................................ 24

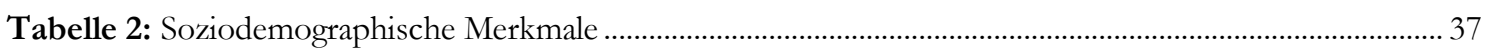

Tabelle 3: Anzahl der ermittelten Begleitdiagnosen pro Probandin der Versuchsgruppen mit einer führend

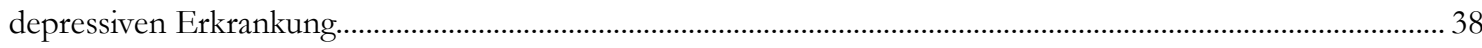

Tabelle 4: Übersicht der eingenommenen somatischen Medikamente................................................................ 40

Tabelle 5: Anzahl der eingenommenen Psychopharmaka für die Versuchsgruppen mit einer führend

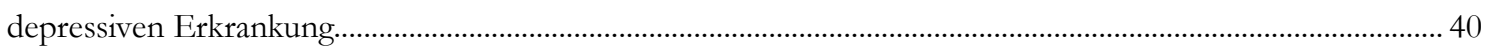

Tabelle 6: Ergebnisse des ETI und CTQ differenziert nach Versuchsgruppe.................................................. 42

Tabelle 7: Ergebnisse der MADRS und des BDI-II differenziert nach Versuchsgruppe.................................. 44

Tabelle 8: Auswertung des TSST: systolische und diastolische Blutdruck- sowie Pulswerte der Baseline,

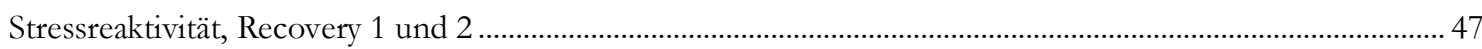

Tabelle 9: Anteil positiver und negativer prozentualer Veränderungen der kardiovaskulären Parameter des sBD, dBD und der HF

Tabelle 10: Gruppenvergleich der Stressreaktivität, Recovery 1 und 2 für die kardiovaskulären Parameter sBD, dBD und HF.

Tabelle 11: Übersicht über die Ergebnisse der Studien zum Einfluss von Depression und Kindheitstraumata auf die kardiovaskuläre Stressreaktivität

Tabelle 12: Übersicht über Ergebnisse der Studien zum Einfluss von Depression und Kindheitstraumata auf die kardiovaskuläre Recovery 69 


\section{Anhang}

Tabelle A1: Exakte p- und t-Werte der T-Tests zur Berechnung der Signifikanz der prozentualen Veränderungen der kardiovaskulären Parameter der Stressreaktivität, Recovery 1 und 2

\begin{tabular}{|c|c|c|c|c|c|c|c|c|c|c|c|c|}
\hline \multirow[b]{2}{*}{$Z^{\text {䍃 }}$} & ${ }_{i}^{5}$ & $\begin{array}{l}\stackrel{\circ}{\circ} \\
+\end{array}$ & $\stackrel{\vec{\nabla}}{\hat{p}}$ & $\stackrel{m}{m}$ & & $\begin{array}{l}\infty \\
\infty \\
\stackrel{0}{n}\end{array}$ & $\stackrel{\overbrace{}}{\rightarrow}$ & $\begin{array}{l}\hat{\theta} \\
\text { in }\end{array}$ & & $\frac{a}{0}$ & $\begin{array}{l}\text { กิ } \\
\text { în }\end{array}$ & 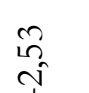 \\
\hline & ب & $\begin{array}{l}\overline{8} \\
8 \\
8 \\
0\end{array}$ & $\begin{array}{l}\overrightarrow{5} \\
\stackrel{8}{8} \\
\stackrel{0}{0}\end{array}$ & 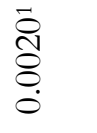 & & $\begin{array}{l}\text { है } \\
\stackrel{8}{8} \\
8 \\
0\end{array}$ & 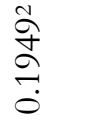 & $\begin{array}{l}\stackrel{y}{\tilde{m}} \\
\stackrel{0}{0} \\
\stackrel{0}{0}\end{array}$ & & $\begin{array}{l}\text { 管 } \\
\text { 定 } \\
0 \\
0\end{array}$ & $\begin{array}{l}\stackrel{2}{\hat{n}} \\
\stackrel{n}{0} \\
\stackrel{0}{0}\end{array}$ & $\begin{array}{l}\stackrel{n}{0} \\
\stackrel{0}{0} \\
\dot{0}\end{array}$ \\
\hline \multirow[b]{2}{*}{ 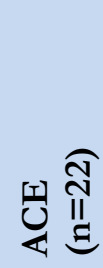 } & $\frac{\Delta}{\Delta}$ & $\stackrel{n}{\stackrel{9}{c}}$ & $\stackrel{\circ}{\circ}$ & $\stackrel{N}{\approx}$ & & $\overrightarrow{\hat{N}_{0}}$ & $\stackrel{n}{\stackrel{n}{i}}$ & $\begin{array}{l}\text { ô } \\
\infty_{0}\end{array}$ & & $\cong$ & 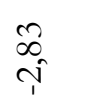 & $\stackrel{+}{+}$ \\
\hline & 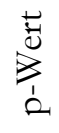 & $\begin{array}{l}\text { तิ } \\
\text { ปै. } \\
\stackrel{0}{0}\end{array}$ & 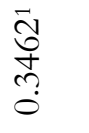 & $\begin{array}{l}\overline{0} \\
\infty \\
0 \\
0 \\
0\end{array}$ & & 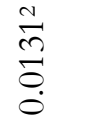 & 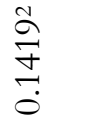 & 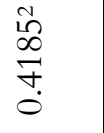 & & $\begin{array}{l}\text { 䈝 } \\
\stackrel{n}{0} \\
\stackrel{0}{0}\end{array}$ & 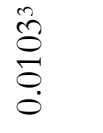 & $\begin{array}{l}\stackrel{n}{n} \\
\infty \\
\stackrel{1}{0} \\
0 \\
0\end{array}$ \\
\hline \multirow{3}{*}{ 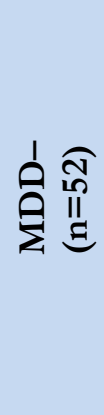 } & ${ }_{1}^{ \pm}$ & 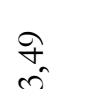 & $\begin{array}{l}\text { ర్ } \\
\sim\end{array}$ & $\stackrel{n}{\stackrel{n}{0}}$ & & $\stackrel{0}{\stackrel{2}{2}}$ & $\hat{\sigma}$ & $\stackrel{?}{m}$ & & 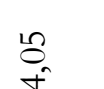 & $\underset{\sigma}{\hat{\sigma}}$ & $\hat{n}$ \\
\hline & $\begin{array}{l}\frac{ \pm}{0} \\
\vdots \\
\vdots \\
\vdots\end{array}$ & $\begin{array}{l}\stackrel{0}{0} \\
\dot{8} \\
\stackrel{0}{0}\end{array}$ & $\begin{array}{l}\overrightarrow{\tilde{o}} \\
\stackrel{0}{0} \\
\dot{0}\end{array}$ & $\begin{array}{l}\overrightarrow{\vec{n}} \\
\stackrel{n}{q} \\
\stackrel{0}{0}\end{array}$ & & $\begin{array}{l}\text { Iै } \\
\stackrel{8}{8} \\
8 \\
0\end{array}$ & $\begin{array}{l}\underset{\Xi}{\Xi} \\
\stackrel{\Xi}{0}\end{array}$ & 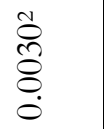 & & 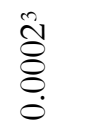 & $\begin{array}{l}\frac{3}{0} \\
\frac{8}{8} \\
0\end{array}$ & $\frac{\tilde{n}}{\stackrel{2}{*}}$ \\
\hline & ${ }_{\frac{1}{0}}^{0}$ & $\stackrel{5}{\stackrel{5}{\sigma}}$ & $\overrightarrow{\sigma_{n}}$ & $g_{-}$ & & $\begin{array}{c}\stackrel{n}{n} \\
\text { mn } \\
m\end{array}$ & $\begin{array}{l}\infty \\
0 \\
\sim \\
1\end{array}$ & ڤn & & $\stackrel{+}{\sim}$ & $\stackrel{\infty}{\stackrel{\infty}{\sim}}$ & $\overrightarrow{\vec{\sigma}_{0}}$ \\
\hline 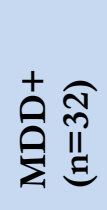 & $\begin{array}{l}\frac{ \pm}{0} \\
\vdots \\
0\end{array}$ & $\begin{array}{l}\overrightarrow{\widehat{d}} \\
\delta \\
\delta \\
\dot{0}\end{array}$ & $\begin{array}{l}\overrightarrow{\tilde{8}} \\
\stackrel{8}{8} \\
\dot{0}\end{array}$ & 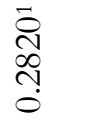 & & $\stackrel{\frac{\pi}{8}}{\frac{8}{8}}$ & \begin{tabular}{l}
\multirow{0}{0}{} \\
$\stackrel{0}{0}$ \\
0
\end{tabular} & 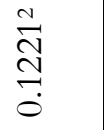 & & 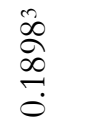 & 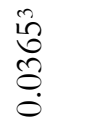 & 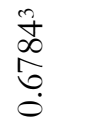 \\
\hline \multirow{3}{*}{ 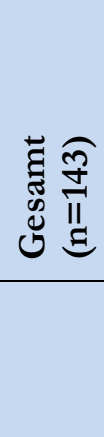 } & $\frac{0}{0}$ & $\stackrel{2}{\curvearrowright}$ & $\begin{array}{l}0 \\
0 \\
i n\end{array}$ & 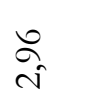 & & $\hat{n}$ & \begin{tabular}{l}
$f$ \\
\multirow{r}{*}{}
\end{tabular} & $\begin{array}{l}\tilde{O} \\
{ }_{n}\end{array}$ & & $\stackrel{+}{m}$ & $\begin{array}{l}\stackrel{0}{1} \\
\text { n̂́ }\end{array}$ & 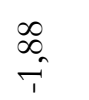 \\
\hline & 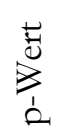 & $\begin{array}{l}8 \\
8 \\
8 \\
0\end{array}$ & $\begin{array}{l}8 \\
8 \\
8 \\
0\end{array}$ & $\begin{array}{l}\stackrel{1}{2} \\
\stackrel{8}{8} \\
\dot{0}\end{array}$ & & $\begin{array}{l}8 \\
8 \\
8 \\
\stackrel{0}{0}\end{array}$ & $\begin{array}{l}\infty \\
\stackrel{8}{8} \\
8 \\
0\end{array}$ & \begin{tabular}{l}
8 \\
8 \\
8 \\
\hdashline
\end{tabular} & & $\begin{array}{l}\vec{\Xi} \\
\stackrel{8}{0}\end{array}$ & \begin{tabular}{l}
8 \\
8 \\
8 \\
\hdashline
\end{tabular} & $\stackrel{a}{\vec{\sigma}}$ \\
\hline & क्ञ & 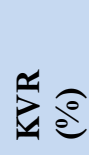 & 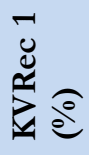 & $\sum_{N}^{0}$ & 의 & 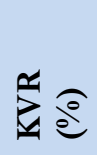 & 苞 & 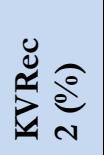 & $\underline{\mathbf{I}}$ & $\stackrel{1}{a} \odot$ & 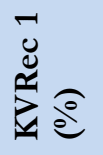 & 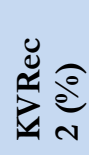 \\
\hline
\end{tabular}

Reaktivität: prozentuale Differenz der kardiovaskulären Parameter von t2 (vor dem Stress) $\rightarrow$ t3 (direk.t nach dem TSST), Recovery 1: prozentuale Differenz der kardiovaskulären Parameter von t3 (direkt nach dem TSST) $\rightarrow$ t4 (nach der Erholungsphase), Recovery 2: prozentuale Differenz der kardiovaskulären Parameter im Vergleich von t2 (vor dem Stress) $\rightarrow$ t4 (nach der Erholungsphase). Adjustierung des Signifikeanzniveaus nach Bonferroni-Holm: ${ }^{1} p \leq 0.007,{ }^{2} p \leq 0.003,{ }^{3} p \leq 0.01$ 
Tabelle A2: Durchschnittsalter der Probanden sowie psychometrische Instrumente in vergleichbaren

Studien

\begin{tabular}{|c|c|c|}
\hline Studie & $\begin{array}{c}\text { Durchschnitts- } \\
\text { alter } \\
\text { (in Jahren) }\end{array}$ & $\begin{array}{c}\text { Instrument zur } \\
\text { Erfassung von } \\
\text { MDD/ACE }\end{array}$ \\
\hline Matthews 2005 & $18-50$ & $\mathrm{CES} \mathrm{D}^{1}$ \\
\hline Guinjoan 1995 & $\sim 42$ & DSM-III-R $^{2}$ \\
\hline Heim $2000^{3}$ & $\sim 31$ & SKID, ETI \\
\hline Taylor 2006 & $\sim 62$ & $\mathrm{DISH}^{3}$ \\
\hline Hamer 2007 & 23,4 & CES-D \\
\hline Carrol 2007 & 42,3 & $\mathrm{HADS}^{4}$ \\
\hline York 2007 & 63 & BDI \\
\hline Salomon 2009 & 31,65 & SKID-I, BDI-II \\
\hline De Rooij 2009 & 58,3 & HADS \\
\hline Schwerdtfeger 2011 & 22,95 & BDI \\
\hline Brindle 2013 & $\sim 20$ & BDI, CES-D $^{1}$ \\
\hline $\begin{array}{l}\text { Yuenyongchaiwat } \\
2017\end{array}$ & 31,52 & HADS \\
\hline Otte 2005 & 28 & SKID, LSC-R ${ }^{5}$ \\
\hline Lovallo 2012 & 24 & $\begin{array}{c}\text { Self-report Items, } \\
\text { BDI }\end{array}$ \\
\hline Gooding 2014 & 14,93 & CTQ \\
\hline Voellmin 2015 & 21,7 & SKID, ETI \\
\hline
\end{tabular}

${ }^{1}$ CES-D: Center for Epidemiological Studies- Depression Scale; ${ }^{2}$ DSM-III-R: Diagnostic and Statistical Manual of Mental Disorders-III-Revisited; ${ }^{3}$ DISH: Hamilton Depression Interview, ${ }^{4} \mathrm{H} A D S$ : Hamilton Anxiety and Depression Scale, ${ }^{5}$ LSCR: Lifestressor Checklist- Revisited, ${ }^{6}$ MSTT: Mirror Star Tracing Task, ${ }^{7}$ PASAT: Paced Auditory Serial Addition Test, ${ }^{8}$ MIST: Montreal Imaging Stress Task 


\section{$9 \quad$ Literaturverzeichnis}

Afifi TO, Mota N, MacMillan HL, Sareen J (2013): Harsh Physical Punishment in Childhood and Adult Physical Health. Pediatrics $\underline{132}$, 333-340

Afifi TO, MacMillan HL, Boyle M, Cheung K, Taillieu T, Turner S, Sareen J (2016): Child abuse and physical health in adulthood. Health Rep $\underline{27}, 10-18$

Allen MT, Matthews KA, Sherman FS (1997): Cardiovascular reactivity to stress and left ventricular mass in youth. Hypertension $\underline{30}, 782-787$

Anda RF, Croft JB, Felitti VJ, Nordenberg D, Giles WH, Williamson DF, Giovino GA (1999): Adverse childhood experiences and smoking during adolescence and adulthood. JAMA 282, 1652 1658

Anda RF, Brown DW, Felitti VJ, Bremner JD, Dube SR, Giles WH (2007): Adverse childhood experiences and prescribed psychotropic medications in adults. Am J Prev Med $\underline{32}$, 389-394

Anda RF, Brown DW, Dube SR, Bremner JD, Felitti VJ, Giles WH (2008a): Adverse childhood experiences and chronic obstructive pulmonary disease in adults. Am J Prev Med $\underline{34}, 396-403$

Anda RF, Brown DW, Felitti VJ, Dube SR, Giles WH (2008b): Adverse childhood experiences and prescription drug use in a cohort study of adult HMO patients. BMC Public Health $\underline{8}, 198$

Andrews J, Pruessner JC (2013): The combined propranolol/TSST paradigm: a new method for psychoneuroendocrinology. PLoS One $\underline{8}$, e 57567

https://doi.org/10.1371/journal.pone.0057567 (abgerufen am 16.09.2017)

Andrews J, D’Aguiar C, Pruessner JC (2012): The Combined Dexamethasone/TSST Paradigm: a New Method for Psychoneuroendocrinology. PLoS ONE 7, e38994

https://doi.org/10.1371/journal.pone.0038994 (abgerufen am 16.09.2017)

AWMF-Leitlinie (2015): Unipolare Depression. Arbeitsgemeinschaft der Wissenschaftlichen Medizinischen Fachgesellschaften (AWMF)- Ständige Kommission Leitlinien S3-Leitlinie/Nationale Versorgungsleitlinie Unipolare Depression Langfassung https://www.awmf.org/uploads/tx_szleitlinien/nvl-0051_S3_Unipolare_Depression_2017-05.pdf (abgerufen am 19.08.2019) 
Bairey Merz CN, Kop W, Krantz DS, Helmers KF, Berman DS, Rozanski A (1998): Cardiovascular stress response and coronary artery disease: evidence of an adverse postmenopausal effect in women. Am Heart J 135, 881-887

Bali A, Jaggi AS (2015): Clinical experimental stress studies: methods and assessment. Rev Neurosci $\underline{26}, 555-579$

Balsamo M (2010): Anger and depression: evidence of a possible mediating role for rumination. Psychol Rep 106, 3-12

Barth J, Schneider S, von Känel R (2010): Lack of social support in the etiology and the prognosis of coronary heart disease: a systematic review and meta-analysis. Psychosom Med $\underline{72}, 229-238$

Batten SV, Aslan M, Maciejewski PK, Mazure CM (2004): Childhood maltreatment as a risk factor for adult cardiovascular disease and depression. J Clin Psychiatry $\underline{65}, 249-254$

Beck AT, Ward CH, Mendelson M, Mock J, Erbaught J (1961): An Inventory for Measuring Depression. Arch Gen Psychiatry 4, 561-571

Beck AT, Steer RA, Brown GK: Manual for the Beck Depression Inventory-II. Psychological corporation, San Antonio 1996

Becker M, Schulz A: Epidemiologie von Kindesmisshandlung. In: Spitzer C, Grabe HJ (Hrsg.): Kindesmisshandlung- psychische und körperliche Folgen im Erwachsenenalter. Kohlhammer, Stuttgart 2013, 13-22

Bentley T, Widom CS (2009): A 30-year follow-up of the effects of child abuse and neglect on obesity in adulthood. Obes Res $\underline{17}, 1900-1905$

Bernstein DP, Fink L: Childhood Trauma Questionnaire: A retrospective self-report questionnaire and manual. Pearson Assessment, San Antonio 1998

Bernstein DP, Fink L, Handelsman L, Foote J, Lovejoy M, Wenzel K, Sapareto E, Ruggiero J (1994): Initial reliability and validity of a new retrospective measure of child abuse and neglect. Am J Psychiatry 151, 1132-1136 
Birkett MA (2011): The Trier Social Stress Test protocol for inducing psychological stress. J Vis Exp 드, e3238

https://doi.org/10.3791/3238 (abgerufen am 12.05.2017)

Bornschein G: Vergleich der physiologischen Stressreagibilität von Frauen mit komplexen Traumafolgestörungen und gesunden Frauen. Med. Diss. Göttingen 2014

Bortz J, Schuster C: Statistik für Human- und Sozialwissenschaftler. 7. Auflage; Springer-Verlag, Berlin 2010

Bosch NM, Riese H, Reijneveld SA, Bakker MP, Verhulst FC, Ormel J, Oldehinkel AJ (2012): Timing matters: long term effects of adversities from prenatal period up to adolescence on adolescents' cortisol stress response. The TRAILS study. Psychoneuroendocrinology $\underline{37}, 1439-1447$

Bradley RG, Binder EB, Epstein MP, Tang Y, Nair HP, Liu W, Gillespie CF, Berg T, Evces M, Newport DJ, et al. (2008): Influence of child abuse on adult depression: moderation by the corticotropin-releasing hormone receptor gene. Arch Gen Psychiatry $\underline{65}$, 190-200

Bremner JD, Vermetten E, Mazure CM (2000): Development and preliminary psychometric properties of an instrument for the measurement of childhood trauma: the Early Trauma Inventory. Depress Anxiety 12, 1-12

Brindle RC, Ginty AT, Conklin SM (2013): Is the association between depression and blunted cardiovascular stress reactions mediated by perceptions of stress? Int J Psychophysiol $\underline{90}, 66-72$

Brosschot JF, Gerin W, Thayer JF (2006): The perseverative cognition hypothesis: a review of worry, prolonged stress-related physiological activation, and health. J Psychosom Res $\underline{60}, 113-124$

Busch LY, Possel P, Valentine JC (2017): Meta-analyses of cardiovascular reactivity to rumination: A possible mechanism linking depression and hostility to cardiovascular disease. Psychol Bull $\underline{143}$, 1378-1394

Busch MA, Maske UE, Ryl L, Schlack R, Hapke U (2013): Prävalenz von depressiver Symptomatik und diagnostizierter Depression bei Erwachsenen in Deutschland: Ergebnisse der Studie zur Gesundheit Erwachsener in Deutschland (DEGS1). Bundesgesundheitsbl 56, 733-739 
Cannon WB (1929): Organisation for physiological homeostasis. Physiol Rev 9, 399-431

Cannon WB: Wut, Hunger, Angst und Schmerz - Eine Physiologie der Emotionen. Übersetzt von Helmut Junker. Urban \& Schwarzenberg, München 1975

Carretero OA, Oparil S (2000): Essential hypertension- Part I: definition and etiology. Circulation 101, 329-335

Carroll D, Phillips AC, Hunt K, Der G (2007): Symptoms of depression and cardiovascular reactions to acute psychological stress: evidence from a population study. Biol Psychol $\underline{75}$, 68-74

Carroll D, Phillips AC, Der G, Hunt K, Benzeval M (2011): Blood pressure reactions to acute mental stress and future blood pressure status: data from the 12-year follow-up of the West of Scotland Study. Psychosom Med $\underline{73}, 737-742$

Carroll D, Ginty AT, Der G, Hunt K, Benzeval M, Phillips AC (2012): Increased blood pressure reactions to acute mental stress are associated with. Psychophysiology 49, 1444-1448

Chapman DP, Whitfield CL, Felitti VJ, Dube SR, Edwards VJ, Anda RF (2004): Adverse childhood experiences and the risk of depressive disorders in adulthood. J Affect Disord 82, 217-225

Chartier MJ, Walker JR, Naimark B (2009): Health risk behaviors and mental health problems as mediators of the relationship between childhood abuse and adult health. Am J Public Health $\underline{99}$, $847-854$

Chiang JJ, Bower JE, Irwin MR, Taylor SE, Fuligni AJ (2017): Adiposity moderates links from early adversity and depressive symptoms to inflammatory reactivity to acute stress during late adolescence. Brain Behav Immun $\underline{66}, 146-155$

Chida Y, Steptoe A (2010): Greater cardiovascular responses to laboratory mental stress are associated with poor subsequent cardiovascular risk status: a meta-analysis of prospective evidence. Hypertension $\underline{55}, 1026-1032$

Chrousos GP (2009): Stress and disorders of the stress system. Nat Rev Endocrinol $\underline{5}$, 374-381

Chrousos GP, Gold PW (1992): The concepts of stress and stress system disorders. Overview of physical and behavioral homeostasis. JAMA $\underline{267}, 1244-1252$ 
Colquhoun DM, Bunker SJ, Clarke DM, Glozier N, Hare DL, Hickie IB, Tatoulis J, Thompson DR, Tofler GH, Wilson A, Branagan MG (2013): Screening, referral and treatment for depression in patients with coronary heart disease. Med J Aust 198, 483-484

Comijs HC, Beekman ATF, Smit F, Bremmer M, van Tilburg T, Deeg DJH (2007): Childhood adversity, recent life events and depression in late life. J Affect Disord 103, 243-246

Conraads VM, Deaton C, Piotrowicz E, Santaularia N, Tierney S, Piepoli MF, Pieske B, Schmid JP, Dickstein K, Ponikowski PP, Jaarsma T (2012): Adherence of heart failure patients to exercisebarriers and possible solutions: a position statement of the Study Group on Exercise Training in Heart Failure of the Heart Failure Association of the European Society of Cardiology. Eur J Heart Fail $14,451-458$

de Rooij SR, Schene AH, Phillips DI, Roseboom TJ (2010): Depression and anxiety: Associations with biological and perceived stress reactivity to a psychological stress protocol in a middle-aged population. Psychoneuroendocrinology $\underline{35}, 866-877$

Dedovic K, Renwick R, Mahani NK, Engert V, Lupien SJ, Pruessner JC (2005): The Montreal Imaging Stress Task: using functional imaging to investigate the effects of perceiving and processing psychosocial stress in the human brain. J Psychiatry Neurosci $\underline{30}, 319-325$

Dickerson SS, Kemeny ME (2004): Acute stressors and cortisol responses: a theoretical integration and synthesis of laboratory research. Psychol Bull 130, 355-391

Dong M, Giles WH, Felitti VJ, Dube SR, Williams JE, Chapman DP, Anda RF (2004): Insights into causal pathways for ischemic heart disease: adverse childhood experiences study. Circulation $\underline{110}$, $1761-1766$

Dube SR, Anda RF, Felitti VJ, Chapman DP, Williamson DF, Giles WH (2001): Childhood abuse, household dysfunction, and the risk of attempted suicide throughout the life span: findings from the Adverse Childhood Experiences Study. JAMA 286, 3089-3096

Dube SR, Miller JW, Brown DW, Giles WH, Felitti VJ, Dong M, Anda RF (2006): Adverse childhood experiences and the association with ever using alcohol and initiating alcohol use during adolescence. J Adolesc Health $\underline{38}$, 444.e1-444.e10 https://doi.org/10.1016/j.jadohealth.2005.06.006 (abgerufen am 23.03.2018) 
Edwards KM, Mills PJ: Interaktion zwischen dem endokrinen, dem zentralnervösen und dem Immunsystem. In: Ehlert U, von Känel R (Hrsg.): Psychoendokrinologie und Psychoimmunologie. Springer-Verlag, Heidelberg 2011, 111-127

Edwards VJ, Holden GW, Felitti VJ, Anda RF (2003): Relationship between multiple forms of childhood maltreatment and adult mental health in community respondents: results from the adverse childhood experiences study. Am J Psychiatry 160, 1453-1460

Ehlert U: Das endokrine System. In: Ehlert U, von Känel R (Hrsg.): Psychoendokrinologie und Psychoimmunologie. Springer-Verlag, Heidelberg 2011, 3-36

Ehmke H: Das Kreislaufsystem. In: Pape HC, Kurtz A, Silbernagl S (Hrsg.): Physiologie. 7. Auflage; Thieme-Verlag, Stuttgart 2014, 214-266

Everson SA, Kaplan GA, Goldberg DE, Salonen JT (1996): Anticipatory blood pressure response to exercise predicts future high blood pressure in middle-aged men. Hypertension 27, 1059-1064

Everson SA, Lynch JW, Chesney MA, Kaplan GA, Goldberg DE, Shade SB, Cohen RD, Salonen R, Salonen JT (1997): Interaction of workplace demands and cardiovascular reactivity in progression of carotid atherosclerosis: population based study. BMJ $\underline{314}, 553-558$

Faith MS, Matz PE, Jorge MA (2002): Obesity- depression associations in the population. J Psychosom Res $\underline{53}, 935-942$

Felitti VJ, Anda RF, Nordenberg D, Williamson DF, Spitz AM, Edwards V, Koss MP, Marks JS (1998): Relationship of childhood abuse and household dysfunction to many of the leading causes of death in adults. The Adverse Childhood Experiences (ACE) Study. Am J Prev Med 14, 245-258

Fergusson DM, Boden JM, Horwood LJ (2008): Exposure to childhood sexual and physical abuse and adjustment in early adulthood. Child Abuse Negl $\underline{32}, 607-619$

Fergusson DM, McLeod GFH, Horwood LJ (2013): Childhood sexual abuse and adult developmental outcomes: findings from a 30-year longitudinal study in New Zealand. Child Abuse Negl $\underline{37}$, 664-674

Frasure-Smith N, Lesperance F (2005): Reflections on depression as a cardiac risk factor. Psychosom Med 67, 19-25 
Fu ML (1995): Do immune system changes have a role in hypertension? J Hypertens 13 , 1259-1265

Fydrich T, Renneberg B, Schmitz B, Wittchen H-U: SKID II. Strukturiertes Klinisches Interview für DSM-IV. Achse II: Persönlichkeitsstörungen. Interviewheft. Hogrefe-Verlag, Göttingen 1997

Garad Y, Maximova K, MacKinnon N, McGrath JJ, Kozyrskyj AL, Colman I (2017): Sex-Specific Differences in the Association Between Childhood Adversity and Cardiovascular Disease in Adulthood: Evidence From a National Cohort Study. Can J Cardiol 33, 1013-1019

Gilbert R, Widom CS, Browne K, Fergusson D, Webb E, Janson S (2009): Burden and consequences of child maltreatment in high-income countries. Lancet $\underline{373}$, 68-81

Ginty AT, Carroll D, Roseboom TJ, Phillips AC, de Rooij SR (2013): Depression and anxiety are associated with a diagnosis of hypertension 5 years later in a cohort of late middle-aged men and women. J Hum Hypertens $\underline{27}$, 187-190

Gold SM, Zakowski SG, Valdimarsdottir HB, Bovbjerg DH (2004): Higher Beck depression scores predict delayed epinephrine recovery after acute psychological stress independent of baseline levels of stress and mood. Biol Psychol $\underline{67}, 261-273$

Gooding HC, Milliren CE, Austin SB, Sheridan MA, McLaughlin KA (2016): Child Abuse, Resting Blood Pressure, and Blood Pressure Reactivity to Psychosocial Stress. J Pediatr Psychol 41, 5-14

Grabe HJ, Schwahn C, Appel K, Mahler J, Schulz A, Spitzer C, Fenske K, Barnow S, Lucht M, Freyberger HJ (2010): Childhood maltreatment, the corticotropin-releasing hormone receptor gene and adult depression in the general population. Am J Med Genet Part B $\underline{153 \mathrm{~B}}, 1483-1493$

Grabe HJ, Mahler J, Becker M: Frühe Traumatisierung und affektive Störungen. In: Spitzer C, Grabe HJ (Hrsg.): Kindesmisshandlung- psychische und körperliche Folgen im Erwachsenenalter. Kohlhammer, Stuttgart 2013, 146-161

Gronwall, D, Sampson, H: The psychological effects of concussion. Auckland University Press, Auckland 1974

Guinjoan SM, Bernabó JL, Cardinali DP (1995): Cardiovascular tests of autonomic function and sympathetic skin responses in patients with major depression. J Neurol Neurosurg Psychiatry $\underline{59}$, 299-302 
Hamer M, Tanaka G, Okamura H, Tsuda A, Steptoe A (2007): The effects of depressive symptoms on cardiovascular and catecholamine responses to the induction of depressive mood. Biol Psychol 74, 20-25

Handwerker HO, Kress M: Koordination spezieller Organfunktionen- Vegetatives Nervensystem. In: Speckmann EJ, Hescheler J, Köhling R (Hrsg.): Physiologie. 6. Auflage; Elsevier, München 2013, 663-739

Hardt J, Vellaisamy P, Schoon I (2010): Sequelae of prospective versus retrospective reports of adverse childhood experiences. Psychol Rep 107, 425-440

Häuser W, Schmutzer G, Brähler E, Glaesmer H (2011): Misshandlungen in Kindheit und JugendErgebnisse einer Umfrage in einer repräsentativen Stichprobe der deutschen Bevölkerung. Dtsch Arztebl Int 108, 287-294

Hautzinger M, Keller F, Kühner C: Beck Depressions-Inventar - Revision (BDI-II). 2. Auflage; Pearson Assessment, Frankfurt am Main 2009

Heim C, Newport DJ, Heit S, Graham YP, Wilcox M, Bonsall R, Miller AH, Nemeroff CB (2000): Pituitary-adrenal and autonomic responses to stress in women after sexual and physical abuse in childhood. JAMA $\underline{284}$, 592-597

Heim C, Newport DJ, Mletzko T, Miller AH, Nemeroff CB (2008): The link between childhood trauma and depression: insights from HPA axis studies in humans. Psychoneuroendocrinology $\underline{33}$, 693-710

Herrmann-Lingen C (2000): Biopsychosoziale Faktoren in Genese und Maifestation der Koronaren Herzkrankheit. Z Psychosom Med Psychother 46, 315-330

Herrmann-Lingen C, Lomb J, Schunk T (2010): Multidimensionality of mental stress-induced autonomic activation in a psychosomatic patient population. Psychosom Med $\underline{72}$, A-128

Het S, Rohleder N, Schoofs D, Kirschbaum C, Wolf OT (2009): Neuroendocrine and psychometric evaluation of a placebo version of the „Trier Social Stress Test“. Psychoneuroendocrinology $\underline{34}$, 1075-1086

Hillis SD, Anda RF, Felitti VJ, Marchbanks PA (2001): Adverse childhood experiences and sexual risk behaviors in women: a retrospective cohort study. Fam Plann Perspect 33, 206-211 
Hirokawa K, Nagayoshi M, Ohira T, Kajiura M, Kitamura A, Kiyama M, Okada T, Iso H (2014): Menopausal status in relation to cardiovascular stress reactivity in healthy Japanese participants. Psychosom Med 무, 701-708

Huppelsberg J, Walther K: Hormone. In: Huppelsberg J, Walther K (Hrsg.): Kurzlehrbuch Physiologie. 3. Auflage; Thieme-Verlag, Stuttgart 2009, 195-210

ICD-10-GM 2019- Systematisches Verzeichnis. Internationale statistische Klassifikation der Krankheiten und verwandter Gesundheitsprobleme (10. Revision, German Modification). O. Verf., bearbeitet von Graubner B, hrsg. v. Deutschen Institut für Medizinische Dokumentation und Information (DIMDI) unter Beteiligung der Arbeitsgruppe ICD des Kuratoriums für Fragen der Klassifikation im Gesundheitswesen (KKG). Deutscher Ärzteverlag, Köln 2018

Ikin JF, Creamer MC, Sim MR, McKenzie DP (2010): Comorbidity of PTSD and depression in Korean War veterans: prevalence, predictors, and impairment. J Affect Disord 125, 279-286

Jacobi F, Hofler M, Siegert J, Mack S, Gerschler A, Scholl L, Busch MA, Hapke U, Maske U, Seiffert I (2014): Twelve-month prevalence, comorbidity and correlates of mental disorders in Germany: the Mental Health Module of the German Health Interview and Examination Survey for Adults (DEGS1-MH). Int J Methods Psychiatr Res 23, 304-319

Jennings JR, Kamarck TW, Everson-Rose SA, Kaplan GA, Manuck SB, Salonen JT (2004): Exaggerated blood pressure responses during mental stress are prospectively related to enhanced carotid atherosclerosis in middle-aged Finnish men. Circulation 110, 2198-2203

Jezova D, Makatsori A, Duncko R, Moncek F, Jakubek M (2004): High trait anxiety in healthy subjects is associated with low neuroendocrine activity during psychosocial stress. Prog Neuropsychopharmacol Biol Psychiatry 28, 1331-1336

Jönsson P, Wallergård M, Österberg K, Hansen ÅM, Johansson G, Karlson B (2010): Cardiovascular and cortisol reactivity and habituation to a virtual reality version of the Trier Social Stress Test: A pilot study. Psychoneuroendocrinology $\underline{35}, 1397-1403$

Kaplan MS, Nunes A (2003): The psychosocial determinants of hypertension. Nutr Metab Cardiovasc Dis $\underline{13}, 52-59$

Karlin WA, Brondolo E, Schwartz J (2003): Workplace social support and ambulatory cardiovascular activity in New York City traffic agents. Psychosom Med 65, 167-176 
Kendler KS, Bulik CM, Silberg J, Hettema JM, Myers J, Prescott CA (2000): Childhood sexual abuse and adult psychiatric and substance use disorders in women: an epidemiological and cotwin con-

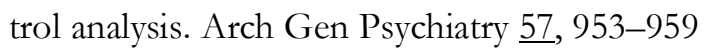

Kibler JL, Ma M (2004): Depressive symptoms and cardiovascular reactivity to laboratory behavioral stress. Int J Behav Med $\underline{11}, 81-87$

Kinzie JD, Riley C, McFarland B, Hayes M, Boehnlein J, Leung P, Adams G (2008): High prevalence rates of diabetes and hypertension among refugee psychiatric patients. J Nerv Ment Dis 196 , $108-112$

Kirschbaum C, Pirke KM, Hellhammer DH (1993): The „Trier Social Stress Test“- a tool for investigating psychobiological stress responses in a laboratory setting. Neuropsychobiology $\underline{28}, 76-81$

Klinitzke G, Rompell M, Häuser W, Brähler E, Glaesmer H (2012): Die deutsche Version des Childhood Trauma Questionnaire (CTQ) - psychometrische Eigenschaften in einer bevölkerungsrepräsentativen Stichprobe. Psychother Psych Med 62, 47-51

Koch C, Leinweber B, Drengberg B, Blaum C, Oster H (2017): Interaction between circadian rhythms and stress. Neurobiol Stress $\underline{6}, 57-67$

Koob GF, Le Moal M (2001): Drug addiction, dysregulation of reward, and allostasis. Neuropsychopharmacology $\underline{24}, 97-129$

Krefetz DG, Steer RA, Gulab NA, Beck AT (2002): Convergent validity of the Beck depression inventory-II with the reynolds adolescent depression scale in psychiatric inpatients. J Pers Assess $\underline{78}, 451-460$

Krishnaveni GV, Jones A, Veena SR, Somashekara R, Karat SC, D Fall CH (2018): Adiposity and Cortisol Response to Stress in Indian Adolescents. Indian Pediatr $\underline{55}, 125-130$

Kudielka BM, Buske-Kirschbaum A, Hellhammer DH, Kirschbaum C (2004): Differential heart rate reactivity and recovery after psychosocial stress (TSST) in healthy children, younger adults, and elderly adults: the impact of age and gender. Int J Behav Med 11, 116-121

Kudielka BM, Hellhammer DH, Kirschbaum C: Ten years of research with the trier social stress test- revisited. In: Harmon-Jones E, Vinkielman P (Hrsg.): Social Neuroscience: Integrating Biological and Psychological Explanations of Social Behavior. New York 2007, 56-83 
Kuehl LK, Muhtz C, Hinkelmann K, Dettenborn L, Wingenfeld K, Spitzer C, Otte C (2016): Association between major depression and cardiovascular risk: the role of antidepressant medication. Psychopharmacology 233, 3289-3295

Ladwig KH, Lederbogen F, Albus C, Angermann C, Borggrefe, M, Fischer D, Fritzsche K, Haass M, Jordan J, Jünger J, Kindermann I, Köllner V, Kuhn B, Scherer M, Seyfarth M, Völler H, Waller C, Herrmann-Lingen C (2013): Positionspapier zur Bedeutung von psychosozialen Faktoren in der Kardiologie- Update 2013. Kardiologe 7, 7-27

La Rovere MT, Mortara A, Schwartz PJ (1995): Baroreflex sensitivity. J Cardiovasc Electrophysiol $\underline{6}, 761-774$

La Rovere MT, Pinna GD, Raczak G (2008): Baroreflex sensitivity: measurement and clinical implications. Ann Noninvasive Electrocardiol $\underline{13}, 191-207$

Lewington S, Clarke R, Qizilbash N, Peto R, Collins R (2002): Age-specific relevance of usual blood pressure to vascular mortality: a meta-analysis of individual data for one million adults in 61 prospective studies. Lancet $\underline{360}, 1903-1913$

Light KC, Kothandapani RV, Allen MT (1998): Enhanced cardiovascular and catecholamine responses in women with depressive symptoms. Int J Psychophysiol 28, 157-166

Lindert J, von Ehrenstein OS, Grashow R, Gal G, Braehler E, Weisskopf MG (2014): Sexual and physical abuse in childhood is associated with depression and anxiety over the life course: systematic review and meta-analysis. Int J Public Health $\underline{59}$, 359-372

Lobbestael J, Leurgans M, Arntz A (2011): Inter-rater reliability of the Structured Clinical Interview for DSM-IV Axis I Disorders (SCID I) and Axis II Disorders (SCID II). Clin Psychol Psychother $\underline{18}, 75-79$

Loucks EB, Almeida ND, Taylor SE, Matthews KA (2011): Childhood family psychosocial environment and coronary heart disease risk. Psychosom Med $\underline{73}, 563-571$

Lovallo WR (2011): Do low levels of stress reactivity signal poor states of health? Biol Psychol $\underline{86}$, $121-128$

Lovallo WR, Gerin W (2003): Psychophysiological reactivity: mechanisms and pathways to cardiovascular disease. Psychosom Med 65, 36-45 
Lovallo WR, Farag NH, Sorocco KH, Cohoon AJ, Vincent AS (2012): Lifetime Adversity Leads to Blunted Stress Axis Reactivity: Studies from the Oklahoma Family Health Patterns Project. Biol Psychiatry $\underline{71}, 344-349$

Low CA, Thurston RC, Matthews KA (2010): Psychosocial factors in the development of heart disease in women: current research and future directions. Psychosom Med $\underline{72}, 842-854$

Maercker A, Michael T, Fehm L, Becker ES, Margraf J (2004): Age of traumatisation as a predictor of post-traumatic stress disorder or major depression in young women. Br J Psychiatry $\underline{184}, 482$ 487

Maffei C, Fossati A, Agostoni I, Barraco A, Bagnato M, Deborah D, Namia C, Novella L, Petrachi M (1997): Interrater reliability and internal consistency of the structured clinical interview for DSMIV axis II personality disorders (SCID-II), version 2.0. J Personal Disord 11, 279-284

Maier W, Philipp M (1985): Comparative analysis of observer depression scales. Acta Psychiatr Scand $\underline{72}$, 239-245

Markovitz JH, Raczynski JM, Wallace D, Chettur V, Chesney MA (1998): Cardiovascular reactivity to video game predicts subsequent blood pressure increases in young men: The CARDIA study. Psychosom Med 60, 186-191

Matthews KA, Katholi CR, McCreath H, Whooley MA, Williams DR, Zhu S, Markovitz JH (2004): Blood pressure reactivity to psychological stress predicts hypertension in the CARDIA study. Circulation $\underline{110}, 74-78$

Matthews SC, Nelesen RA, Dimsdale JE (2005): Depressive symptoms are associated with increased systemic vascular resistance to stress. Psychosom Med 67, 509-513

McElroy SL, Kotwal R, Malhotra S, Nelson EB, Keck PE, Nemeroff CB (2004): Are mood disorders and obesity related? A review for the mental health professional. J Clin Psychiatry 65 , 634-651

McEwen BS (1998): Stress, adaptation, and disease. Allostasis and allostatic load. Ann N Y Acad Sci $\underline{840}, 33-44$

McEwen BS (2002): Sex, stress and the hippocampus: allostasis, allostatic load and the aging process. Neurobiol Aging $\underline{23}, 921-939$ 
McEwen BS, Seeman T (1999): Protective and damaging effects of mediators of stress. Elaborating and testing the concepts of allostasis and allostatic load. Ann N Y Acad Sci $\underline{896}, 30-47$

Meng L, Chen D, Yang Y, Zheng Y, Hui R (2012): Depression increases the risk of hypertension incidence: a meta-analysis of prospective cohort studies. J Hypertens $\underline{30}, 842-851$

Meyer T, Albrecht J, Bornschein G, Sachsse U, Herrmann-Lingen C (2016): Posttraumatic Stress Disorder (PTSD) Patients Exhibit a Blunted Parasympathetic Response to an Emotional Stressor. Appl Psychophysiol Biofeedback 41, 395-404

Montgomery S, Åsberg M (1979): A new depression scale designed to be sensitive to change. Br J Psychiatry $\underline{134}, 382-389$

Muller MJ, Szegedi A, Wetzel H, Benkert O (2000): Moderate and severe depression. Gradations for the Montgomery-Asberg Depression Rating Scale. J Affect Disord 무, 137-140

Newman JD, McGarvey ST, Steele MS (1999): Longitudinal association of cardiovascular reactivity and blood pressure in Samoan adolescents. Psychosom Med 61, 243-249

Nolen-Hoeksema S (2000): The role of rumination in depressive disorders and mixed anxiety/depressive symptoms. J Abnorm Psychol 109, 504-511

Otte C, Neylan TC, Pole N, Metzler T, Best S, Henn-Haase C, Yehuda R, Marmar CR (2005): Association between childhood trauma and catecholamine response to psychological stress in police academy recruits. Biol Psychiatry $\underline{57}, 27-32$

Pacak K, Palkovits M, Kopin IJ, Goldstein DS (1995): Stress-induced norepinephrine release in the hypothalamic paraventricular nucleus and pituitary-adrenocortical and sympathoadrenal activity: in vivo microdialysis studies. Front Neuroendocrinol 16, 89-150

Pan A, Sun Q, Okereke OI, Rexrode KM, Hu FB (2011): Depression and risk of stroke morbidity and mortality: a meta-analysis and systematic review. JAMA $\underline{306}$, 1241-1249

Park S, Hong JP, Bae JN, Cho S-J, Lee D-W, Lee J-Y, Chang SM, Jeon HJ, Hahm B-J, Lee YM (2014): Impact of childhood exposure to psychological trauma on the risk of psychiatric disorders and somatic discomfort: single vs. multiple types of psychological trauma. Psychiatry Res 219, $443-$ 449 
Paschke R: Endokrines System. In: Pape HC, Kurtz A, Silbernagl S (Hrsg.): Physiologie. 7. Auflage; Thieme Verlag, Stuttgart 2014, 586-635

Pearson TA, Mensah GA, Alexander RW, Anderson JL, Cannon RO 3rd, Criqui M, Fadl YY, Fortmann SP, Hong Y, Myers GL, et al. (2003): Markers of inflammation and cardiovascular disease: application to clinical and public health practice: A statement for healthcare professionals from the Centers for Disease Control and Prevention and the American Heart Association. Circulation 107, 499-511

Phillips AC, Ginty AT, Hughes BM (2013): The other side of the coin: blunted cardiovascular and cortisol reactivity are associated with negative health outcomes. Int J Psychophysiol $\underline{90,1-7}$

Pickering TG (2007): Stress, inflammation, and hypertension. J Clin Hypertens 9, 567-571

Putnam FW (2003): Ten-year research update review: child sexual abuse. J Am Acad Child Adolesc Psychiatry $\underline{42}, 269-278$

Raikkonen K, Matthews KA, Flory JD, Owens JF (1999): Effects of hostility on ambulatory blood pressure and mood during daily living in healthy adults. Health Psychol $\underline{18}, 44-53$

Rettig R, Kuschinsky W: Herz-Kreislauf-Funktion. In: Speckmann EJ, Hescheler J, Köhling R (Hrsg.): Physiologie. 6. Auflage; Elsevier, München 2013, 377-435

Riley E, Wright R, Jun H, Hibert E, Rich-Edwards J (2010): Hypertension in Adult Survivors of Child Abuse: Observations from the Nurses' Health Study II. J Epidemiol Community Health $\underline{64}$, 413-418

Robert Koch-Institut (Hrsg): Gesundheit in Deutschland. Gesundheitsberichterstattung des Bundes. Gemeinsam getragen von RKI und Destatis. RKI, Berlin 2015 https://doi.org/10.17886/rkipubl-2015-003 (abgerufen am 10.08.2017)

Roemmich JN, Lobarinas CL, Joseph PN, Lambiase MJ, Archer FD, Dorn J (2009): Cardiovascular reactivity to psychological stress and carotid intima-media thickness in children. Psychophysiology 46, 293-299

Roemmich JN, Feda DM, Seelbinder AM, Lambiase MJ, Kala GK, Dorn J (2011): Stress-induced cardiovascular reactivity and atherogenesis in adolescents. Atherosclerosis $\underline{215}, 465-470$ 
Rooks C, Veledar E, Goldberg J, Votaw J, Shah A, Bremner JD, Vaccarino V (2015): Long-Term Consequences of Early Trauma on Coronary Heart Disease: Role of Familial Factors. J Trauma Stress 28, 456-459

Rosengren A, Hawken S, Ounpuu S, Sliwa K, Zubaid M, Almahmeed WA, Blackett KN, Sitthiamorn C, Sato H, Yusuf S (2004): Association of psychosocial risk factors with risk of acute myocardial infarction in 11119 cases and 13648 controls from 52 countries (the INTERHEART study): case-control study. Lancet $\underline{364}, 953-962$

Rozanski A, Blumenthal JA, Davidson KW, Saab PG, Kubzansky L (2005): The epidemiology, pathophysiology, and management of psychosocial risk factors in cardiac practice: the emerging field of behavioral cardiology. J Am Coll Cardiol 45, 637-651

Salomon K, Clift A, Karlsdottir M, Rottenberg J (2009): Major depressive disorder is associated with attenuated cardiovascular reactivity and impaired recovery among those free of cardiovascular disease. Health Psychol $\underline{28}, 157-165$

Salomon K, Bylsma LM, White KE, Panaite V, Rottenberg J (2013): Is blunted cardiovascular reactivity in depression mood-state dependent? A comparison of major depressive disorder remitted depression and healthy controls. Int J Psychophysiol 90, 50-57

Sato N, Miyake S (2004): Cardiovascular reactivity to mental stress: relationship with menstrual cycle and gender. J Physiol Anthropol Appl Human Sci $\underline{23}$, 215-223

Schallmayer S, Hughes BM (2010): Impact of oral contraception and neuroticism on cardiovascular stress reactivity across the menstrual cycle. Psychol Health Med $\underline{15}, 105-115$

Schmidtke A, Fleckenstein P, Moises W, Beckmann H (1988): Studies of the reliability and validity of the German version of the Montgomery-Asberg Depression Rating Scale (MADRS). Schweiz Arch Neurol Psychiatr (1985) 139, 51-65

Schoedl AF, Costa MCP, Mari JJ, Mello MF, Tyrka AR, Carpenter LL, Price LH (2010): The clinical correlates of reported childhood sexual abuse: an association between age at trauma onset and severity of depression and PTSD in adults. J Child Sex Abuse 19, 156-170

Schuler JL, O’Brien WH (1997): Cardiovascular recovery from stress and hypertension risk factors: a meta-analytic review. Psychophysiology $\underline{34}$, 649-659 
Schulkin J (1999): Corticotropin-releasing hormone signals adversity in both the placenta and the brain: regulation by glucocorticoids and allostatic overload. J Endocrinol 161, 349-356

Schwartz AR, Gerin W, Davidson KW, Pickering TG, Brosschot JF, Thayer JF, Christenfeld N, Linden W (2003): Toward a causal model of cardiovascular responses to stress and the development of cardiovascular disease. Psychosom Med $\underline{65}, 22-35$

Schwerdtfeger A, Rosenkaimer AK (2011): Depressive symptoms and attenuated physiological reactivity to laboratory stressors. Biol Psychol $\underline{87}, 430-438$

Scott KM, Von Korff M, Angermeyer MC, Benjet C, Bruffaerts R, de Girolamo G, Haro JM, Lepine JP, Ormel J, Posada-Villa J (2011): Association of childhood adversities and early-onset mental disorders with adult-onset chronic physical conditions. Arch Gen Psychiatry $\underline{68}$, 838-844

Seeman TE, Singer BH, Rowe JW, Horwitz RI, McEwen BS (1997): Price of adaptation- allostatic load and its health consequences. MacArthur studies of successful aging. Arch Intern Med 157, 2259-2268

Selye H (1936): A Syndrome Produced by Diverse Nocuous Agents. Nature 138, 32

Selye H (1950): Stress and the General Adaptation Syndrome. Br Med J 1, 1383-1392

Selye H (1975): Confusion and Controversy in the Stress Field. J Human Stress 1, 37-44

Singh K, Shen BJ (2013): Abdominal obesity and chronic stress interact to predict blunted cardiovascular reactivity. Int J Psychophysiol 무, 73-79

Spasojevic J, Alloy LB (2001): Rumination as a common mechanism relating depressive risk factors to depression. Emotion 1, 25-37

Spitzer C, Dümpelmann M: Dissoziative und somatoforme Störungen. In: Spitzer C, Grabe HJ (Hrsg.): Kindesmisshandlung- psychische und körperliche Folgen im Erwachsenenalter. Kohlhammer, Stuttgart 2013, 161-181 
Spitzer C, Wingenfeld K: Stressregulation durch Nahrungsaufnahme? Die Relevanz einer veränderten Regulation der Hypothalamus-Hypophysen-Nebennierenrinden Achse in Folge lebensgeschichtlich früher Traumatisierungen. DFG-Sachbeihilfe-Antrag (Projektnummer 216932859), Deutsche Forschungsgemeinschaft 2012 https://gepris.dfg.de/gepris/projekt/216932859 (abgerufen am 19.08.2019)

Spitzer C, Hinkelmann K, Otte C: Kardiovaskuläre Erkrankungen. In: Spitzer C, Grabe HJ (Hrsg.): Kindesmisshandlung - psychische und körperliche Folgen im Erwachsenenalter. Kohlhammer, Stuttgart 2013, 205-218

Spitzer C, Meyer T, Herrmann-Lingen C (2016): Komplexe Traumatisierungen und körperliche Gesundheit- Assoziation von Kindesmisshandlungen und koronarer Herzkrankheit. Psychotherapeut 61, 191-196

Spitzer C, Barnow S, Volzke H, John U, Freyberger HJ, Grabe HJ (2009): Trauma, posttraumatic stress disorder, and physical illness: findings from the general population. Psychosom Med $\underline{71}$, 1012-1017

Spitzer C, Otte C, Kuehl LK, May A, Schultebraucks K, Hellmann-Regen J, Wingenfeld K (2018): The dexamethasone corticotropin releasing hormone test in healthy and depressed women with and without childhood adversity. Psychoneuroendocrinology $\underline{87}, 147-151$

Springer KW, Sheridan J, Kuo D, Carnes M (2007): Long-term physical and mental health consequences of childhood physical abuse: results from a large population-based sample of men and women. Child Abuse Negl $\underline{31}, 517-530$

Steptoe A, Marmot M (2005): Impaired cardiovascular recovery following stress predicts 3-year increases in blood pressure. J Hypertens $\underline{23}, 529-536$

Steptoe A, Marmot M (2006): Psychosocial, hemostatic, and inflammatory correlates of delayed poststress blood pressure recovery. Psychosom Med $\underline{68}, 531-537$

Sterling P, Eyer J: Allostasis: A new paradigm to explain arousal pathology. In: Fisher S, Reason J (Hrsg.): Handbook of life stress, cognition, and health. John Wiley and Sons, New York 1988, 629649

Stewart JC, Janicki DL, Kamarck TW (2006): Cardiovascular reactivity to and recovery from psychological challenge as predictors of 3-year change in blood pressure. Health Psychol 25, 111-118 
Straznicky NE, Barrington VE, Branley P, Louis WJ (1998): A study of the interactive effects of oral contraceptive use and dietary fat intake on blood pressure, cardiovascular reactivity and glucose tolerance in normotensive women. J Hypertens 16 , 357-368

Taylor CB, Conrad A, Wilhelm FH, Neri E, DeLorenzo A, Kramer MA, Giese-Davis J, Roth WT, Oka R, Cooke JP (2006): Psychophysiological and cortisol responses to psychological stress in depressed and nondepressed older men and women with elevated cardiovascular disease risk. Psychosom Med $\underline{68}, 538-546$

Terock J, Van der Auwera S, Janowitz D, Spitzer C, Barnow S, Miertsch M, Freyberger HJ, Grabe HJ (2016): From Childhood Trauma to Adult Dissociation: The Role of PTSD and Alexithymia. Psychopathology 49, 374-382

Treiber FA, Musante L, Kapuku G, Davis C, Litaker M, Davis H (2001): Cardiovascular (CV) responsivity and recovery to acute stress and future $\mathrm{CV}$ functioning in youth with family histories of CV disease: a 4-year longitudinal study. Int J Psychophysiol 41, 65-74

Treiber FA, Kamarck T, Schneiderman N, Sheffield D, Kapuku G, Taylor T (2003): Cardiovascular reactivity and development of preclinical and clinical disease states. Psychosom Med $\underline{65}$, 46-62

Ulrich-Lai YM, Herman JP (2009): Neural regulation of endocrine and autonomic stress responses. Nat Rev Neurosci 10, 397-409

Voellmin A, Winzeler K, Hug E, Wilhelm FH, Schaefer V, Gaab J, La Marca R, Pruessner JC, Bader K (2015): Blunted endocrine and cardiovascular reactivity in young healthy women reporting a history of childhood adversity. Psychoneuroendocrinology $\underline{51}, 58-67$

Vonderlin R, Kleindienst N, Alpers GW, Bohus M, Lyssenko L, Schmahl C (2018): Dissociation in victims of childhood abuse or neglect: a meta-analytic review. Psychol Med $\underline{48}, 1-10$

von Känel R (2008): Psychological distress and cardiovascular risk: what are the links? J Am Coll Cardiol $\underline{52}, 2163-2165$

von Känel R: Kardiovaskuläre Krankheiten. In: Ehlert U, von Känel R (Hrsg.): Psychoendokrinologie und Psychoimmunologie. Springer-Verlag, Heidelberg 2011, 267-289

Weiß C: Basiswissen Medizinische Statistik. 6. Auflage; Springer-Verlag, Berlin 2013 
WHO (1999a): 1999 World Health Organization-International Society of Hypertension Guidelines for the Management of Hypertension. Guidelines Subcommittee. J Hypertens 17, 151-183

WHO (1999b): Report of the Consultation on Child Abuse Prevention. Violence and Injury Prevention Team \& Global Forum for Health Research. Genf 29-31 March 1999

http://www.who.int/iris/handle/10665/65900 (abgerufen am 16.09.2017)

Whooley MA (2006): Depression and cardiovascular disease: healing the broken-hearted. JAMA $\underline{295}, 2874-2881$

Whooley MA, Wong JM (2013): Depression and cardiovascular disorders. Annu Rev Clin Psychol 9, 327-354

Williams JBW, Kobak KA (2008): Development and reliability of a structured interview guide for the Montgomery Asberg Depression Rating Scale (SIGMA). Br J Psychiatry 192, 52-58

Williamson DF, Thompson TJ, Anda RF, Dietz WH, Felitti V (2002): Body weight and obesity in adults and self-reported abuse in childhood. Int J Obes Relat Metab Disord 26, 1075-1082

Wingenfeld K, Spitzer C, Mensebach C, Grabe HJ, Hill A, Gast U, Schlosser N, Höpp H, Beblo T, Driessen M (2010): Die deutsche Version des Childhood Trauma Questionnaire (CTQ): Erste Befunde zu den psychometrischen Kennwerten. Psychother Psychosom Med Psychol 60, 442-450

Wingenfeld K, Driessen M, Mensebach C, Rullkoetter N, Schaffrath C, Spitzer C, Schlosser N, Beblo T, Heim C (2011): Die deutsche Version des „Early Trauma Inventory“ (ETI) - Erste psychometrische Charakterisierung eines Interviews zur Erfassung traumatischer Lebensereignisse in der Kindheit und Jugend. Diagnostica $\underline{57}, 27-38$

Wingenfeld K, Grabe HJ, Spitzer C: Diagnostik biographisch früher Traumatisierung. In: Spitzer C, Grabe HJ (Hrsg.): Kindesmisshandlung- psychische und körperliche Folgen im Erwachsenenalter. Kohlhammer, Stuttgart 2013, 103-118

Wingenfeld K, Kuehl LK, Boeker A, Schultebraucks K, Schulz A, Stenzel J, Spitzer C, Otte C (2017a): Are adverse childhood experiences and depression associated with impaired glucose tolerance in females? An experimental study. J Psychiatr Res $\underline{95}$, 60-67 
Wingenfeld K, Kuehl LK, Boeker A, Schultebraucks K, Ritter K, Hellmann-Regen J, Otte C, Spitzer C (2017b): Stress reactivity and its effects on subsequent food intake in depressed and healthy women with and without adverse childhood experiences. Psychoneuroendocrinology $\underline{80}, 122-130$

Witt A, Sachser C, Plener PL, Brähler E, Fegert JM (2019): Prävalenz und Folgen belastender Kindheitserlebnisse in der deutschen Bevölkerung. Dtsch Arztebl Int 116, 635-642

Saß H, Wittchen HU, Zaudig M, Houben I: Diagnostisches und Statistisches Manual Psychischer Störungen - Textrevision - (DSM-IV-TR). Hogrefe, Göttingen 2003

York KM, Hassan M, Li Q, Li H, Fillingim RB, Sheps DS (2007): Coronary artery disease and depression: patients with more depressive symptoms have lower cardiovascular reactivity during laboratory-induced mental stress. Psychosom Med 69, 521-528

Yuenyongchaiwat K, Baker IS, Sheffield D (2017): Symptoms of anxiety and depression are related to cardiovascular responses to active, but not passive, coping tasks. Rev Bras Psiquiatr $\underline{39}, 110-117$

Yusuf S, Hawken S, Ounpuu S, Dans T, Avezum A, Lanas F, McQueen M, Budaj A, Pais P, Varigos J, Lisheng L (2004): Effect of potentially modifiable risk factors associated with myocardial infarction in 52 countries (the INTERHEART study): case-control study. Lancet 364, 937-952

Zaba M, Kirmeier T, Ionescu IA, Wollweber B, Buell DR, Gall-Kleebach DJ, Schubert CF, Novak B, Huber C, Kohler K, et al. (2015): Identification and characterization of HPA-axis reactivity endophenotypes in a cohort of female PTSD patients. Psychoneuroendocrinology $\underline{55}, 102-115$

Zanarini MC, Frankenburg FR (2001): Attainment and maintenance of reliability of axis I and II disorders over the course of a longitudinal study. Compr Psychiatry $\underline{42}$, 369-374

Zanstra YJ, Johnston DW (2011): Cardiovascular reactivity in real life settings: measurement, mechanisms and meaning. Biol Psychol $\underline{\text { 86, }}, 98-105$ 


\section{Danksagung}

Eine Dissertation ist nicht das Werk eines Einzelnen, darum möchte ich mich an dieser Stelle bei allen Menschen bedanken, die mich in dieser herausfordernden und gleichzeitig sehr wertvollen Phase meiner akademischen Laufbahn unterstützt haben.

Zunächst möchte ich allen Probandinnen und Versuchsleiterinnen und -leitern danken, ohne deren Engagement diese Arbeit nicht möglich gewesen wäre.

Mein besonderer Dank gilt meinem Doktorvater, Herrn Prof. Dr. Carsten Spitzer, für die konstruktive wissenschaftliche Begleitung dieser Dissertation sowie für seine bestärkenden Worte, die mir immer wieder Mut für dieses große Projekt gemacht haben. Herrn Prof. Dr. Christoph Herrmann-Lingen gebührt herzlicher Dank für die Mitbetreuung dieser Arbeit durch methodische und statistische Anregungen.

Meinen geschätzten Freunden und wissenschaftlichen Kollegen Daniel Erdmann und Alexandra Glück danke ich für geistreiche Diskussionen und Ratschläge. 